Geometry 8 Topology

Volume 9 (2005) 403-482

Published: 9 March 2005

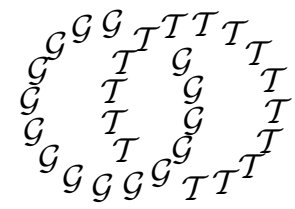

\title{
Flows and joins of metric spaces
}

\author{
IGOR Mineyev \\ Department of Mathematics, University of Illinois at Urbana-Champaign \\ 250 Altgeld Hall, 1409 W Green Street, Urbana, IL 61801, USA
}

Email: mineyev@math.uiuc.edu

\begin{abstract}
We introduce the functor $\otimes$ which assigns to every metric space $X$ its symmetric join $\otimes X$. As a set, $\otimes X$ is a union of intervals connecting ordered pairs of points in $X$. Topologically, $\otimes X$ is a natural quotient of the usual join of $X$ with itself. We define an $\operatorname{Isom}(X)$-invariant metric $d_{*}$ on $\otimes X$.

Classical concepts known for $\mathbb{H}^{n}$ and negatively curved manifolds are defined in a precise way for any hyperbolic complex $X$, for example for a Cayley graph of a Gromov hyperbolic group. We define a double difference, a cross-ratio and horofunctions in the compactification $\bar{X}=X \sqcup \partial X$. They are continuous, $\operatorname{Isom}(X)$-invariant, and satisfy sharp identities. We characterize the translation length of a hyperbolic isometry $g \in \operatorname{Isom}(X)$.

For any hyperbolic complex $X$, the symmetric join $\otimes \bar{X}$ of $\bar{X}$ and the (generalized) metric $d_{*}$ on it are defined. The geodesic flow space $\mathcal{F}(X)$ arises as a part of $\otimes \bar{X}$. $\left(\mathcal{F}(X), d_{*}\right)$ is an analogue of (the total space of) the unit tangent bundle on a simply connected negatively curved manifold. This flow space is defined for any hyperbolic complex $X$ and has sharp properties. We also give a construction of the asymmetric join $X \circledast Y$ of two metric spaces.
\end{abstract}

These concepts are canonical, i.e. functorial in $X$, and involve no "quasi"-language. Applications and relation to the Borel conjecture and others are discussed.

AMS Classification numbers Primary: 20F65, 20F67, 37D40, 51F99, 57Q05

Secondary: $57 \mathrm{M} 07,57 \mathrm{~N} 16,57 \mathrm{Q} 91,05 \mathrm{C} 25$

Keywords: Symmetric join, asymmetric join, metric join, Gromov hyperbolic space, hyperbolic complex, geodesic flow, translation length, geodesic, metric geometry, double difference, cross-ratio

Proposed: Walter Neumann

Seconded: Martin Bridson, David Gabai

Received: 29 July 2004

Revised: 17 February 2005

(C) Geometry $8 \mathcal{T}$ Topology Publications 


\section{Introduction}

Let $X$ be a proper geodesic hyperbolic metric space in the sense of Gromov. In [30, the following discrete-continuous dichotomy was shown for a non-elementary closed subgroup $H<\operatorname{Isom}(X)$ acting cocompactly on $X$ : either

- $H$ has a proper non-elementary vertex-transitive action on a hyperbolic graph of bounded valency, or

- there is a finite-index open subgroup $H^{*}<H$ and a compact normal subgroup $K \triangleleft H$ contained in $H^{*}$ such that $H^{*} / K$ is a connected simple Lie group of rank one.

This says, less formally, that to understand general hyperbolic spaces it suffices to study hyperbolic graphs and Lie groups. While the theory of Lie groups and symmetric spaces is quite developed, the hyperbolic graphs and groups introduced by Gromov 27 are relatively recent phenomena. By its very nature of being discrete a hyperbolic graph lacks a nice local structure, and therefore the tools of differential geometry.

In this paper we fill in the gaps in the discrete spaces and the blanks in the discrete spaces theory. The main philosophical point is that hyperbolic groups, despite being discrete, do give rise to many concepts that were known on the continuous side. This paper introduces several sharp geometric concepts first for arbitrary metric spaces, and then for hyperbolic spaces in the sense of Gromov. These concepts in particular eliminate the need of the "quasi"-language when talking about hyperbolic groups and spaces.

We introduce the notion of symmetric join. If $X$ is a set, the symmetric join of $X$, denoted $\otimes X$, is the "obvious" union of formal intervals connecting ordered pairs of points in $X$; the interval connecting a point to itself is required to degenerate. When $X$ is a topological space we define a natural topology on $\otimes X$. When $X$ is a metric space, we define a metric $d_{*}$ on $\otimes X$ with natural properties. The symmetric join is therefore an example of a metric join. Even though $\otimes X$ is an abstract union of intervals, the construction of $d_{*}$ is very explicit. The metric $d_{*}$ is canonical and $\operatorname{Isom}(X)$-invariant.

In 31, the Baum-Connes conjecture was proved for hyperbolic groups and their subgroups by constructing a strongly bolic metric $\hat{d}$ on any hyperbolic group. Having a strongly bolic metric is not sufficient for the constructions of the present paper. We show that (a modified version of) $\hat{d}$ has stronger properties and use it to define the double difference $\langle\cdot, \cdot \mid \cdot, \cdot\rangle$ in $X$ (see 6.2). 
The main property used is that $\hat{d}$ and $\langle\cdot, \cdot \mid \cdot, \cdot\rangle$ behave "exponentially well" at infinity (Theorem 32). We show that the double difference continuously extends to $\bar{X}$ and gives rise to a continuous cross-ratio in $\bar{X}$ (section 7 ). It is the use of the metric $\hat{d}$ that allows things to extend continuously to the boundary. This generalizes the work of Otal [34] who defined and used the cross-ratio for negatively curved manifolds.

The "Hyperbolic groups" article by Gromov [27] was an inspiration for many mathematicians over the last years, including the author of this paper. Gromov outlined a construction of a metric space $\hat{G}$ with $\mathbb{R}-, \mathbb{Z}_{2}-$, and $\Gamma$-actions 27, 8.3.C]. He considers the set of all biinfinite geodesics in the Cayley graph and then identifies those geodesics that connect the same pairs of points in $\partial \Gamma$. Mathéus 29 and Champetier 10 provided further details of the Gromov's construction. The identification of geodesics is by quasiisometries, so $\hat{G}$ is rather a quasigeodesic flow; $\mathbb{R}$ acts on the $\mathbb{R}$-orbits in $\hat{G}$ by quasiisometric homeomorphisms.

In 23] Furman takes $\partial^{2} \Gamma \times \mathbb{R}$ as a model set for the flow space, so geodesics are unique by definition. He uses boundedness of cocycles from [13] to construct a geodesic current, i.e. an invariant measure on $\partial^{2} \Gamma$, then provides a $\Gamma$-action on $\partial^{2} \Gamma \times \mathbb{R}$ and a $\Gamma$-invariant cross-ratio on $\partial \Gamma$. Both the action and the cross-ratio are measure-theoretic, that is defined up to subsets of measure 0 . Bühler [5] considers the space of all geodesics in a hyperbolic space $X$, as in the Gromov's construction, and uses the amenability of the $\Gamma$-action on $\partial \Gamma$ to make a measurable choice of geodesic, i.e. a choice of geodesic for almost every pair of points in $\partial \Gamma$. Since the geodesics in $X$ are chosen in a measurable fashion, and they usually do not depend continuously on their endpoints, there is no obvious way to topologically identify the union of such geodesics with $\partial^{2} \Gamma \times \mathbb{R}$. In both [23] and [5] the space considered is a measure space rather than a metric space. Bourdon [2] presented geodesic flows with sharp properties in the case of $\operatorname{CAT}(-1)$ spaces.

The present paper provides a new approach to constructing a geodesic flow $\mathcal{F}(X)$ for an arbitrary hyperbolic complex $X$, for example when $X$ is a Cayley graph of a hyperbolic group (see 5.3 for definitions). First we enlarge $\otimes X$ to the symmetric join $\otimes \bar{X}$ of the compactification $\bar{X}=X \sqcup \partial X$. We put the metric $\hat{d}$ on $X$ and show that the metric $d_{*}$ canonically determined by $\hat{d}$ extends to $\otimes \bar{X}$ (with the obvious infinite values allowed at infinite points). The use of $\hat{d}$ is essential here. Then $\mathcal{F}(X) \subseteq \otimes \bar{X}$ is by definition the union of lines in $\otimes \bar{X}$ that connect pairs of points in $\partial X$, equipped with the restricted metric $d_{*}$.

Our construction of symmetric join $\oplus \bar{X}$ is more general than the flow space, since it allows for lines to connect points in $X$ as well as points in $\partial X$. But 
even when restricted to $\mathcal{F}(X)$ it provides strong properties (see Theorem [60). Generally speaking, the outcomes of this construction are continuous, rather than measurable as in [23, 5], and sharp, rather than defined "up to a bounded amount" as in [27, 29, 10, 35. Continuity is important for future topological applications. The construction is also more general since we consider an arbitrary hyperbolic complex $X$ with the action of the full simplicial isometry group Isom $(X)$, i.e. the group acting on $X$ does not have to be discrete. The metric $d_{*}$, both on $\mathcal{F}(X)$ and on $\otimes X$, is $\operatorname{Isom}(X)$-invariant. Each $\mathbb{R}$-orbit in $\mathcal{F}(X)$ is an isometric copy of $\mathbb{R}$. $\mathbb{R}$ acts on $\left(\mathcal{F}(X), d_{*}\right)$ by bi-Lipschitz homeomorphisms, and on each $\mathbb{R}$-orbit by isometries in the standard way. The $\mathbb{R}$-orbits in $\mathcal{F}(X)$ converge synchronously and uniformly exponentially.

Symmetric join is a unified concept relating topology and geometry: it combines the usual notion of topological join with the notion of geodesic flow. Symmetric join plays the role of a Riemannian structure on a manifold, and it is canonically assigned to every metric space. When $X$ is a hyperbolic complex, $\otimes \bar{X}$ provides a link between the local and global structures of $X$. This is important, for example, in the study of the topology of manifolds; the manifolds can be chosen to be smooth or not.

For any hyperbolic group $\Gamma$ acting on a hyperbolic complex $X$, for example its Cayley graph, $\mathcal{F}(X)$ provides a convenient model space. It plays the role of (the total space of) the unit tangent bundle over a manifold, even though no manifold was given. There are other models provided by the construction: $\otimes X$, $\otimes \bar{X}, \mathcal{F}(X), X \otimes X, X \otimes \bar{X}, \bar{X} \otimes \bar{X}$, etc (see section [14). They are all equipped with canonical induced $\Gamma$-actions. Their geometry is closely related to the geometry of $\Gamma$ but, unlike $\Gamma$, the spaces are not discrete. It is an interesting question how one can use these models to generalize the Farrell-Jones theory [16, 17, 19, 18, 20, to manifolds with hyperbolic fundamental groups. Also, the asymmetric join of two manifolds might be used in place of a cobordism. One would probably need to generalize the theory of Chapman [11], Ferry [22] and Quinn [36], and to come up with an "h-join theorem" that would play the role of the $\mathrm{h}$-cobordism theorem.

We define continuous horofunctions both in $\bar{X}$ and in $\otimes \bar{X}$ (Theorem [55). They depend only on a point at infinity, rather than on a ray converging to the point (section 10). For each hyperbolic $g \in \operatorname{Isom}(X)$, we define the translation length $\hat{l}(g)$ in terms of metric $\hat{d}$ on $X . \hat{l}(g)$ is indeed realized as the shift of the axis of $g$ in $\mathcal{F}(X)$ (see section 12 and Theorem 60(i)).

Symmetric join is used to provide a notion of join of two metric spaces $Y$ and $Y^{\prime}$, called the asymmetric join $Y \oplus Y^{\prime}$ (section 14). The asymmetric join is 
therefore another example of a metric join. In the case when the two spaces are given an action by the same group, for example when $Y$ and $Y^{\prime}$ are the universal covers of two manifolds with the same fundamental group, we describe a canonical way to put a metric on $Y \otimes Y^{\prime}$ (see 14.1). This situation occurs, for example, in the Borel conjecture that asserts that two closed aspherical manifolds with the same fundamental group are homeomorphic.

If one thinks of the intervals in $Y \otimes Y^{\prime}$ as parameterized by $[-\infty, \infty]$, the set of slices at various times $t \in[-\infty, \infty]$ is a sweep-out, or rather sweep-between, from $Y$ to $Y^{\prime}$. Topologically slices are the same for all $t \in \mathbb{R}$ (they are all homeomorphic to $Y \times Y^{\prime}$ ), but the metric on $Y \otimes Y^{\prime}$ makes it interesting: the slices indeed approach $Y$ as $t \rightarrow-\infty$ and $Y^{\prime}$ as $t \rightarrow \infty$, in a metrically controlled way (Gromov-Hausdorff convergence on bounded subsets). This is of interest in particular in relation with the Borel conjecture. If there exists a homeomorphism between manifolds $M$ and $M^{\prime}$, then it must be present in each slice of $M \oplus M^{\prime}$, diagonally. Since the construction is equivariant, it also allows working with the universal coverings (this gives even more freedom): denote $Y:=\widetilde{M}$ and $Y^{\prime}:=\widetilde{M^{\prime}}$. One needs to find an equivariant copy of $\widetilde{M}$ in each slice of $Y \otimes Y^{\prime}$ (see section 14). Another advantage of using universal coverings is that when $\pi_{1}(M)=\pi_{1}\left(M^{\prime}\right)$ is hyperbolic, the ideal boundary of $Y$ and $Y^{\prime}$ can also be used, since the join of the compactifications $\bar{Y} \otimes \bar{Y}^{\prime}$ is defined as well.

The constructions of this paper require sharp estimates carefully written out. At the first reading, the reader might want to take a look at 2.1 and theorems 32 [35, 40, 45, 55] and 60] which constitute the main results of this paper. Sections 1.15.2 deal with arbitrary metric spaces and simplicial complexes. After that we work in the category of (uniformly locally finite) hyperbolic complexes.

One interesting open problem is to prove the group-theoretic rigidity conjecture, that is the Borel conjecture in the case of hyperbolic fundamental groups. This implies the Poincaré conjecture [21, 19], and it can be viewed as a grouptheoretic (or discrete) analog of the Mostow rigidity theorem.

As we mentioned above, the symmetric join plays the role of a Riemannian structure. Note also that there are examples of closed manifolds with hyperbolic fundamental groups that do not admit a Riemannian structure of negative curvature [15, 12, and our symmetric join construction applies in those cases. Moreover, it applies to all dimensions, and to all PL manifolds that do not admit smooth structure.

Another interesting question is the Cannon's conjecture [6, 9, 8, 7] that a hyperbolic group $\Gamma$ with $\partial \Gamma$ homeomorphic to $S^{2}$ admits a proper cocompact 
action on $\mathbb{H}^{3}$. Note that the boundary of a hyperbolic group is usually very much not a smooth manifold, even if it is a manifold topologically, so one would probably need to use metric geometry rather than the Riemannian one. The essence of all these questions is establishing a link between the local and global structures of hyperbolic metric spaces, and the symmetric flow provides such a link.

The paper is organized as follows. Preliminary definitions are given in section 1 . In section 2 the symmetric join of a metric space $X$ is described. In section 3 we define a metric $d_{*}$ on the symmetric join. In section [4 we discuss the metric and the topology of $X$. In section 5 we define metric complexes and hyperbolic complexes. Section 6 describes a metric $\hat{d}$ and the double difference on a hyperbolic complex $X$. In section 7 cross-ratio on $X$ is defined. In section 8 the symmetric join is extended to $\bar{X}$. Section 9 deals with the topology of the extended symmetric join $\otimes \bar{X}$. In section 10 we define horofunctions and horospheres in $\oplus \bar{X}$. Sections 11 and 12 prove some technical results about convergence of lines and translation length. In section 13 the geodesic flow space is defined and its properties are summarized. Section 14 defines asymmetric join and gives general remarks. The paper ends with an index of symbols and terminology 14.7

The author benefited a lot from helpful conversations with Alex Furman, Misha Gromov, Tadeusz Januszkiewicz, Lowell Jones, Misha Kapovich, Yair Minsky, Frank Quinn and Shmuel Weinberger. Misha Gromov comments that the existence of a continuous cross-ratio and a geodesic flow with sharp properties can be also deduced from the discussion in [27.

The author would like to thank the hospitality of MSRI, Berkeley, in the summer of 2002, of Max-Planck-Institut, Bonn in the summer of 2003, and of IAS, Princeton, in the year 2003-04, where he was supported by NSF grant DMS0111298. This project is partially supported by NSF CAREER grant DMS0228910 .

\section{Preliminaries}

\subsection{The double difference and Gromov product}

Let $(X, d)$ be a metric space. The double difference in $X$ is the function $\langle\cdot, \cdot \mid \cdot, \cdot\rangle: X^{4} \rightarrow \mathbb{R}$ defined by

$$
\left\langle a, a^{\prime} \mid b, b^{\prime}\right\rangle:=\frac{1}{2}\left(d(a, b)-d\left(a^{\prime}, b\right)-d\left(a, b^{\prime}\right)+d\left(a^{\prime}, b^{\prime}\right)\right) .
$$


This notion appeared in a paper by Paulin 35] (under the name "cross ratio").

The following properties are immediate from the definition.

- $\left\langle a, a^{\prime} \mid b, b^{\prime}\right\rangle=\left\langle b, b^{\prime} \mid a, a^{\prime}\right\rangle$.

- $\left\langle a, a^{\prime} \mid b, b^{\prime}\right\rangle=-\left\langle a^{\prime}, a \mid b, b^{\prime}\right\rangle=-\left\langle a, a^{\prime} \mid b^{\prime}, b\right\rangle$.

- $\left\langle a, a \mid b, b^{\prime}\right\rangle=0,\left\langle a, a^{\prime} \mid b, b\right\rangle=0$.

- $\left\langle a, a^{\prime} \mid b, b^{\prime}\right\rangle+\left\langle a^{\prime}, a^{\prime \prime} \mid b, b^{\prime}\right\rangle=\left\langle a, a^{\prime \prime} \mid b, b^{\prime}\right\rangle$.

- $\langle a, b \mid c, x\rangle+\langle b, c \mid a, x\rangle+\langle c, a \mid b, x\rangle=0$.

The function

$$
\langle a \mid b\rangle_{c}:=\langle a, c \mid c, b\rangle=\frac{1}{2}(d(a, c)+d(b, c)-d(a, b))
$$

is the Gromov product with respect to metric $d$. The triangle inequality implies $\langle b \mid c\rangle_{a} \geq 0$. The two concepts are related by the formula

$$
\langle a, b \mid x, y\rangle=\langle b \mid x\rangle_{a}-\langle b \mid y\rangle_{a} .
$$

\subsection{Generalized metrics}

We will deal with points at infinity, so it is convenient to extend the class

of metric spaces. A generalized metric space is a topological space $Y$ with a function $d: Y \times Y \rightarrow[0, \infty]$ such that $d(x, y)=d(y, x), d(x, y)=0$ iff $x=y$, and $d(x, z) \leq d(x, y)+d(y, z)$, for all $x, y, z \in Y$. Here we use the conventions $r+\infty=\infty$ and $r \leq \infty$ for every $r \in[0, \infty]$. For $x \in X$, the finite component of $x$ is the set $\{y \in Y \mid d(x, y)<\infty\}$. Obviously, $Y$ is the disjoint union of its finite components, and the restriction of $d$ to each finite component is a metric in the usual sense. Moreover, we require as a part of definition that the topology on each finite component $V$ of $Y$ coincides with the topology induced by the restriction of $d$ to $V$. The function $d$ is called a generalized metric on $Y$. Note that, for a given $d$, there might be many topologies on $Y$ that make $(X, d)$ a generalized metric space.

The main examples to keep in mind:

- Any metric space is a generalized metric space.

- $\overline{\mathbb{R}}:=[-\infty, \infty]$ with the topology of a closed interval and the obvious generalized metric that we will denote $|\cdot|$; that is, $|x-y|$ denotes the (generalized) distance between $x$ and $y$. 
- Given a hyperbolic graph $\mathcal{G},(\overline{\mathcal{G}}, d)$ is a generalized metric space, where $\overline{\mathcal{G}}=\mathcal{G} \sqcup \partial \mathcal{G}$ is the compactification of $\mathcal{G}$ by its ideal boundary and $d$ is the obvious extension of the word metric on $\mathcal{G}$ to $\overline{\mathcal{G}}$ : we have $d(x, y)=\infty$ for any $x \in \partial \mathcal{G}$ and any $y \in \overline{\mathcal{G}} \backslash\{x\}$.

A map $X \rightarrow Y$ between generalized metric spaces is called an isometric embedding if it preserves the generalized metric. Note that an isometric embedding must not be continuous.

\section{$1.3+$ equivalence, ${ }^{\times}$equivalence, ${ }^{x+}$ equivalence}

In this section we introduce convenient equivalence relations of functions that will be used later in the paper.

For subsets $U \subseteq \overline{\mathbb{R}}$ and $V \subseteq \mathbb{R}$, addition and multiplication can be defined in the obvious way:

$$
U+V:=\{u+v \mid u \in U, v \in V\} \quad \text { and } \quad U V:=\{u v \mid u \in U, v \in V\} .
$$

If $U$ or $V$ consists of just one element, then we write the element instead of the set notation.

Let $S$ be any set, and $\varphi$ and $\psi$ be functions from $S$ to $\overline{\mathbb{R}}$. We say that $\varphi$ and $\psi$ are ${ }^{+}$equivalent, denoted $\varphi \widetilde{\psi}$, if there exists $B \in[0, \infty)$ such that $\varphi(s) \in$ $\psi(s)+[-B, B]$ for all $s \in S$. They are ${ }^{\times}$equivalent if there exists $A \in[1, \infty)$ such that $\varphi(s) \in[1 / A, A] \psi(s)$ for all $s \in S$; and they are ${ }^{\times+}$equivalent if there exist $A \in[1, \infty)$ and $B \in[0, \infty)$ such that $\varphi(s) \in[1 / A, A] \psi(s)+[-B, B]$ for all $s \in S$. It is left to the reader to check that these are indeed equivalence relations.

We will say that a map $f:\left(X_{1}, d_{1}\right) \rightarrow\left(X_{2}, d_{2}\right)$ between metric spaces is a ${ }^{+}$map if $d_{1}(x, y)$ and $d_{2}(f(x), f(y))$ are ${ }^{+}$equivalent as functions of $(x, y) \in X_{1} \times X_{1}$. Similarly for ${ }^{\times}$map and ${ }^{x+}$ map.

A ${ }^{+}$map $f:\left(X_{1}, d_{1}\right) \rightarrow\left(X_{2}, d_{2}\right)$ is called a ${ }^{+}$isometry if there exists a ${ }^{+}$map $f:\left(X_{2}, d_{2}\right) \rightarrow\left(X_{1}, d_{1}\right)$ such that $f \circ g$ and $g \circ f$ are ${ }^{+}$equivalent to the identity maps. A ${ }^{\times+}$map $f:\left(X_{1}, d_{1}\right) \rightarrow\left(X_{2}, d_{2}\right)$ is called a ${ }^{\times+}$isometry if there exists a ${ }^{\times+}$map $f:\left(X_{2}, d_{2}\right) \rightarrow\left(X_{1}, d_{1}\right)$ such that $f \circ g$ and $g \circ f$ are ${ }^{+}$equivalent to the identity maps. ${ }^{x+}$ equivalence of metric spaces is the same thing as quasiisometry. Our definitions of equivalences are more general since they allow considering functions with negative or infinite values.

Lemma 1 Suppose that $\varphi, \varphi^{\prime}, \psi, \psi^{\prime}$ take values in $\mathbb{R}$. 


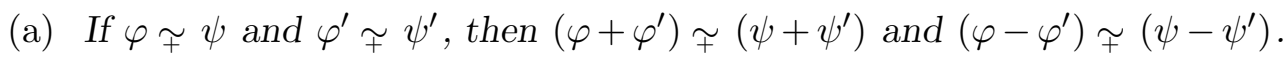

(b) If $\varphi \simeq \psi$, then $|\varphi| \simeq|\psi|$.

The proof follows directly from definitions.

\subsection{Dealing with $\overline{\mathbb{R}}$}

Denote $\overline{\mathbb{R}}:=[-\infty, \infty]$. Throughout the paper we will consider the notions of addition, subtraction, multiplication, taking absolute values and distances in $\overline{\mathbb{R}}$, generalizing the corresponding notions in $\mathbb{R}$. For example,

$$
\begin{gathered}
r+( \pm \infty):= \pm \infty \quad \text { for } \quad r \in \mathbb{R}, \quad( \pm \infty) \cdot l:= \pm \infty \text { for } \quad l \in(0, \infty], \\
\infty-\infty:=0, \quad(-\infty)-(-\infty):=0, \quad|\infty-\infty|:=0, \quad|(-\infty)-(-\infty)|:=0 .
\end{gathered}
$$

Also $e^{\infty}:=\infty$ and $e^{-\infty}:=0$.

\subsection{The smooth-out}

Call a function $\theta: \overline{\mathbb{R}} \rightarrow \overline{\mathbb{R}}$ non-expanding if $|\theta(t)-\theta(s)| \leq|t-s|$ for all $s, t \in \overline{\mathbb{R}}$. Non-expanding functions might be discontinuous: let $\theta$ map $[-\infty, \infty)$ to $[-\infty, 0)$ and $\infty$ to $\infty$.

Lemma 2 Let $\theta: \overline{\mathbb{R}} \rightarrow \overline{\mathbb{R}}$ be a continuous non-expanding non-decreasing function whose image is an interval $[\alpha, \beta] \subseteq \overline{\mathbb{R}}$. Then the function $\theta^{\prime}: \overline{\mathbb{R}} \rightarrow \overline{\mathbb{R}}$,

$$
\theta^{\prime}(t):=\int_{-\infty}^{\infty} \theta(r+t) \frac{e^{-|r|}}{2} d r
$$

is a well-defined continuous non-expanding non-decreasing map from $\overline{\mathbb{R}}$ onto $[\alpha, \beta]$. If, in addition, $\alpha<\beta$, then $\theta^{\prime}$ is an increasing homeomorphism of $\overline{\mathbb{R}}$ onto $[\alpha, \beta]$.

Proof If there exists $t \in \mathbb{R}$ with $\theta(t)=\infty$, then since $\theta$ is non-expanding, $\theta(\mathbb{R})=\{\infty\}$, and since $\theta$ is continuous, $\theta(\overline{\mathbb{R}})=\{\infty\}$. Then $\theta^{\prime}(\overline{\mathbb{R}})=\{\infty\}$ and the lemma holds. Similarly for the case $\theta(t)=-\infty$, so from now on we will assume $\theta(\mathbb{R}) \subseteq \mathbb{R}$. Since $\theta$ is non-expanding, then for each $t \in \mathbb{R}, \theta^{\prime}(t)$ is a well-defined real number. Since $\theta$ is non-decreasing, $\theta(-\infty)=\alpha, \theta(\infty)=\beta$,

$$
\theta^{\prime}(-\infty)=\int_{-\infty}^{\infty} \theta(r-\infty) \frac{e^{-|r|}}{2} d r=\theta(-\infty) \int_{-\infty}^{\infty} \frac{e^{-|r|}}{2} d r=\alpha
$$


and similarly, $\theta^{\prime}(\infty)=\beta$.

$\theta^{\prime}$ is non-decreasing: if $t \leq t^{\prime}$, then for all $r \in \mathbb{R}, r+t \leq r+t^{\prime}, \theta(r+t) \leq \theta\left(r+t^{\prime}\right)$, hence $\theta^{\prime}(t) \leq \theta^{\prime}\left(t^{\prime}\right)$. This implies that $\theta^{\prime}(\overline{\mathbb{R}}) \subseteq[\alpha, \beta]$.

Since $\theta$ is non-expanding,

$$
\begin{gathered}
\left|\theta^{\prime}\left(t^{\prime}\right)-\theta^{\prime}(t)\right| \leq \int_{-\infty}^{\infty}\left|\theta\left(r+t^{\prime}\right)-\theta(r+t)\right| \frac{e^{-|r|}}{2} d r \\
\leq \int_{-\infty}^{\infty}\left|\left(r+t^{\prime}\right)-(r+t)\right| \frac{e^{-|r|}}{2} d r \leq\left|t^{\prime}-t\right|
\end{gathered}
$$

shows that $\theta^{\prime}$ is non-expanding, therefore it is continuous on $\mathbb{R}$.

Now we show the continuity of $\theta^{\prime}$ at $-\infty$. First assume that $\alpha \in \mathbb{R}$. For any $\varepsilon>0$ pick $T \in \mathbb{R}$ such that $\theta(T) \leq \alpha+\varepsilon / 2$ and $R \in[0, \infty)$ such that $\int_{R}^{\infty} r e^{-r} d r=e^{-R}(R+1) \leq \varepsilon$. Since $\theta$ is non-expanding,

$$
\theta(r+t) \leq \theta(t)+|\theta(t+r)-\theta(t)| \leq \theta(t)+|r| .
$$

We claim that $\theta^{\prime}([-\infty, T-R]) \subseteq[\alpha, \alpha+\varepsilon]$. Indeed, for any $t \in[-\infty, T-R]$, since $\theta$ is non-decreasing,

$$
\begin{aligned}
& \theta^{\prime}(t)=\int_{-\infty}^{R} \theta(r+t) \frac{e^{-|r|}}{2} d r+\int_{R}^{\infty} \theta(r+t) \frac{e^{-|r|}}{2} d r \\
& \leq \int_{-\infty}^{R} \theta(R+(T-R)) \frac{e^{-|r|}}{2} d r+\int_{R}^{\infty}(\theta(t)+|r|) \frac{e^{-|r|}}{2} d r \\
& \leq \int_{-\infty}^{R} \theta(T) \frac{e^{-|r|}}{2} d r+\int_{R}^{\infty}(\theta(T)+r) \frac{e^{-r}}{2} d r \\
& =\theta(T)+\int_{R}^{\infty} r \frac{e^{-r}}{2} d r \leq(\alpha+\varepsilon / 2)+\varepsilon / 2=\alpha+\varepsilon
\end{aligned}
$$

This shows the continuity of $\theta^{\prime}$ at $-\infty$ when $\alpha \in \mathbb{R}$. When $\alpha=-\infty$ the argument is similar: for any $N$, pick $T$ so that $\theta(T) \leq N-1$ and let $R=0$, so that $\int_{0}^{\infty} r e^{-r} d x=1$, then deduce that $\theta^{\prime}([-\infty, T]) \subseteq[-\infty, N]$. If $\alpha=\infty$, then since $\theta$ is non-decreasing, $\theta(\overline{\mathbb{R}})=\{\infty\}$; we dealt with this obvious case before. The same argument applies to $\infty$, so $\theta^{\prime}$ is continuous on $\overline{\mathbb{R}}$. This implies that $\theta^{\prime}$ maps $\overline{\mathbb{R}}$ onto $[\alpha, \beta]$.

Now assume $\alpha<\beta$. It remains to show that $\theta^{\prime}$ is strictly increasing, and for that it suffices to show that $\theta^{\prime}$ is such on $\mathbb{R}$. Take any $t, t^{\prime} \in \mathbb{R}$ with $t<t^{\prime}$. Suppose that for all $r \in \mathbb{R}, \theta(r+t)=\theta\left(r+t^{\prime}\right)$. Then $\theta\left(\left(t^{\prime}-t\right)+t\right)=\theta\left(t^{\prime}\right)=\theta(t)$ and inductively, for any positive integer $n$,

$$
\theta\left(n\left(t^{\prime}-t\right)+t\right)=\theta\left((n-1)\left(t^{\prime}-t\right)+t^{\prime}\right)=\theta\left((n-1)\left(t^{\prime}-t\right)+t\right)=\theta(t) .
$$


Since $\theta$ is non-decreasing and continuous, this implies that $\theta$ is a constant function, which is a contradiction with our assumption $\alpha<\beta$. Therefore there exists $r_{0} \in \mathbb{R}$ such that $\theta\left(r_{0}+t^{\prime}\right)>\theta\left(r_{0}+t\right)$. Since $\theta\left(r+t^{\prime}\right)-\theta(r+t) \geq 0$ is continuous in $r$,

$$
\theta^{\prime}\left(t^{\prime}\right)-\theta^{\prime}(t)=\int_{-\infty}^{\infty}\left(\theta\left(r+t^{\prime}\right)-\theta(r+t)\right) \frac{e^{-|r|}}{2} d r>0 .
$$

The lemma says in other words that the "operator" $\theta \mapsto \theta^{\prime}$ makes homeomorphisms out of non-constant continuous monotone functions.

\subsection{Auxiliary functions $\theta$ and $\theta^{\prime}$}

For all $\alpha, \beta \in \overline{\mathbb{R}}$ with $\alpha \leq \beta$ we define a specific function $\theta[\alpha, \beta ; \cdot]: \overline{\mathbb{R}} \rightarrow[\alpha, \beta]$ that mimics projection of one geodesic into another.

$$
\theta[\alpha, \beta ; t]:=\left\{\begin{aligned}
\alpha & \text { if } t \in[-\infty, \alpha] \\
t & \text { if } t \in[\alpha, \beta] \\
\beta & \text { if } t \in[\beta, \infty] .
\end{aligned}\right.
$$

In other words, $\theta[\alpha, \beta ; \cdot]$ is the obvious extension of the identity map $[\alpha, \beta] \rightarrow[\alpha, \beta] . \theta$ satisfies the following properties.

- $\theta$ is continuous in three variables.

- $\theta[\alpha, \beta ; \cdot]$ maps $\overline{\mathbb{R}}$ onto the interval $[\alpha, \beta]$.

- $\theta[\alpha, \beta ; \cdot]$ is non-expanding, that is, $\left|\theta\left[\alpha, \beta ; t_{1}\right]-\theta_{0}\left[\alpha, \beta ; t_{2}\right]\right| \leq\left|t_{1}-t_{2}\right|$.

- $\theta[\alpha, \beta ; t]$ is increasing in $\alpha$ and in $\beta$.

- $\theta[-\infty, \infty ; \cdot]: \overline{\mathbb{R}} \rightarrow \overline{\mathbb{R}}$ is the identity map.

- $\theta$ is shift-invariant: $\theta[\alpha+s, \beta+s ; t+s]=\theta[\alpha, \beta ; t]+s$ for all $s \in \mathbb{R}$.

Define a new function $\theta^{\prime}[\cdot, \cdot ; \cdot]$ as in Lemma 2

$$
\theta^{\prime}[\alpha, \beta ; t]:=\int_{-\infty}^{\infty} \theta[\alpha, \beta ; r+t] \frac{e^{-|r|}}{2} d r
$$

Then $\theta^{\prime}$ satisfies all the above properties of $\theta$ and, in addition,

- for $\alpha<\beta, \theta^{\prime}[\alpha, \beta ; \cdot]$ is a non-expanding homeomorphism of $\overline{\mathbb{R}}$ onto $[\alpha, \beta]$.

The following lemma says that $\theta$ and $\theta^{\prime}$ are close, i.e. the smooth-out does not change functions much.

Lemma $3 \theta^{\prime}[\alpha, \beta ; t]=\theta[\alpha, \beta ; t]+\left(e^{-|t-\alpha|}-e^{-|t-\beta|}\right) / 2 \quad$ for all $\alpha, \beta \in \overline{\mathbb{R}}$, $\alpha \leq \beta, t \in \mathbb{R}$. In particular, $\left|\theta^{\prime}[\alpha, \beta ; t]-\theta[\alpha, \beta ; t]\right| \leq 1$. 
Proof By the definitions of $\theta^{\prime}$ and $\theta$,

$$
\theta^{\prime}[\alpha, \beta ; t]=\int_{-\infty}^{\alpha-t} \alpha \frac{e^{-|r|}}{2} d r+\int_{\alpha-t}^{\beta-t}(r+t) \frac{e^{-|r|}}{2} d r+\int_{\beta-t}^{\infty} \beta \frac{e^{-|r|}}{2} d r .
$$

Now the statement is proved by brute force calculus in each of the following cases: $t \in(-\infty, \alpha], t \in[\alpha, \beta], t \in[\beta, \infty)$.

The derivative of $\theta^{\prime}[\alpha, \beta ; \cdot]$ is obtained by direct calculation:

$$
\frac{\partial}{\partial t} \theta^{\prime}[\alpha, \beta ; t]= \begin{cases}\left(e^{t-\alpha}-e^{t-\beta}\right) / 2 & \text { if } t \in(-\infty, \alpha], \\ 1-\left(e^{\alpha-t}+e^{t-\beta}\right) / 2 & \text { if } t \in[\alpha, \beta], \\ \left(-e^{\alpha-t}+e^{\beta-t}\right) / 2 & \text { if } t \in[\beta, \infty) .\end{cases}
$$

This extends by the same formula to a function

$$
\frac{\partial}{\partial t} \theta^{\prime}[\alpha, \beta ; \cdot]:[-\infty, \infty] \rightarrow[0,1]
$$

The function is increasing on $\left[-\infty, \frac{\alpha+\beta}{2}\right]$ and decreasing on $\left[\frac{\alpha+\beta}{2}, \infty\right]$.

Lemma 4 Suppose that $\alpha, \beta \in \overline{\mathbb{R}}, \alpha \leq \beta, t \in \mathbb{R}$ and $\epsilon \in\left[0,\left(1-e^{\alpha-\beta}\right) / 2\right]$, then

$$
\theta^{\prime}[\alpha, \beta ; t] \in[\alpha+\epsilon, \beta-\epsilon] \quad \Rightarrow \quad \frac{\partial}{\partial t} \theta^{\prime}[\alpha, \beta ; t] \geq \epsilon .
$$

Proof Fix $\alpha, \beta$ and $\epsilon$. If $\beta-\alpha<2 \epsilon$, then the statement is obvious, so we assume $\beta-\alpha \geq 2 \epsilon$. Then we can denote $t_{\epsilon}$ the number such that $\theta^{\prime}\left[\alpha, \beta ; t_{\epsilon}\right]=$ $\beta-\epsilon$. By Lemma 3 and the definition of $\theta$,

$$
\theta^{\prime}[\alpha, \beta ; \beta]=\beta-\left(1-e^{\alpha-\beta}\right) / 2 \leq \beta-\epsilon=\theta^{\prime}\left[\alpha, \beta ; t_{\epsilon}\right] .
$$

Since $\theta^{\prime}[\alpha, \beta ; \cdot]$ is increasing, we have $\beta \leq t_{\epsilon}$. Then by Lemma 3 .

$$
\beta-\epsilon=\theta^{\prime}\left[\alpha, \beta ; t_{\epsilon}\right]=\beta+\left(e^{\alpha-t_{\epsilon}}-e^{\beta-t_{\epsilon}}\right) / 2 \text {, hence }\left(-e^{\alpha-t_{\epsilon}}+e^{\beta-t_{\epsilon}}\right) / 2 \geq \epsilon \text {. }
$$

(The last inequality indeed holds when $\beta=-\infty$ or $\beta=\infty$.) Then by (3),

Similarly,

$$
\frac{\partial}{\partial t} \theta^{\prime}\left[\alpha, \beta ; t_{\epsilon}\right]=\left(-e^{\alpha-t_{\epsilon}}+e^{\beta-t_{\epsilon}}\right) / 2 \geq \epsilon .
$$

$$
\frac{\partial}{\partial t} \theta^{\prime}\left[\alpha, \beta ; s_{\epsilon}\right] \geq \epsilon
$$

where $s_{\epsilon}$ is defined by $\theta^{\prime}\left[\alpha, \beta ; s_{\epsilon}\right]=\alpha+\epsilon$. Now it is a calculus exercise to see from (3) that the minimum of the function $\frac{\partial}{\partial t} \theta^{\prime}[\alpha, \beta ; \cdot]$ on the interval $\left[s_{\epsilon}, t_{\epsilon}\right]$ is attained at the endpoints, so

$$
\frac{\partial}{\partial t} \theta^{\prime}[\alpha, \beta ; t] \geq \epsilon \text { for all } t \in\left[s_{\epsilon}, t_{\epsilon}\right]
$$




\section{$2 \oplus X:$ the symmetric join of $X$}

\subsection{Symmetric join as a topological space}

Given a topological space $X$, its usual topological join (with itself) is the "obvious" union of intervals connecting pairs of points in $X$. Formally, it is the topological space $X \bowtie X:=X^{2} \times \overline{\mathbb{R}} / \sim$, where $(x, y,-\infty) \sim\left(x, y^{\prime},-\infty\right)$ and $(x, y, \infty) \sim\left(x^{\prime}, y, \infty\right)$ for all $x, x^{\prime}, y, y^{\prime} \in X$. We will call the further quotient

$$
\otimes X:=X \bowtie X / \sim
$$

by the equivalence relation $(x, x, s) \sim(x, x, t)$ for all $x \in X, s, t \in \overline{\mathbb{R}}$, the symmetric join of $X$. That is, each interval connecting a point $x \in X$ to itself degenerates to a point in $\otimes X$. The topology on $\otimes X$ is induced by the double quotient $X^{2} \times \overline{\mathbb{R}} \rightarrow X \bowtie X \rightarrow \otimes X$.

Applying the two quotients above in the reverse order gives an equivalent, and more convenient for our purposes, definition of symmetric join:

- denote $\diamond X:=\bigsqcup\{[a, b] \mid a, b \in X\}$, where $[a, b]$ is a topological copy of $\overline{\mathbb{R}}$ if $a \neq b$ and a point if $a=b$;

- let $\otimes X$ be the quotient of $\diamond X$ by the equivalence relation identifying the left endpoint of each $[a, b]$ with $[a, a]$ and the right endpoint with $[b, b]$.

The topology on $\oplus X$ is induced by the obvious double surjection

$$
X^{2} \times \overline{\mathbb{R}} \rightarrow \diamond X \rightarrow \otimes X
$$

in which the lines $\{a\} \times\{b\} \times \overline{\mathbb{R}}$ map onto intervals $[a, b]$.

The canonical embedding $X \hookrightarrow \oplus X, a \mapsto[a, a]$, is a homeomorphism onto its image. We will identify $X$ with its image in $\oplus X$. The sets $\{a\} \times\{b\} \times \overline{\mathbb{R}}$ in $X^{2} \times \overline{\mathbb{R}}$, and their images in $X \bowtie X, \diamond X$, and $\otimes X$, will be called lines. In particular, each point $a \in X$ is the line $[a, a]$. Denote

$$
* X:=\otimes X \backslash X \subseteq \circledast X .
$$

This is the open symmetric join of $X$. The topology on $* X$ is induced from its inclusion into $\otimes X$. Let $\Delta$ be the diagonal in $X^{2}$. The above double surjection restricts to a bijection $\left(X^{2} \backslash \Delta\right) \times \mathbb{R} \rightarrow * X$, so $* X$ is topologically $\left(X^{2} \backslash \Delta\right) \times \mathbb{R}$.

When $X$ is a metric space, we will define actions by $\mathbb{R}, \mathbb{Z}_{2}$ and $\operatorname{Isom}(X)$ on $\otimes X$, and equip $\otimes X$ with a $\mathbb{Z}_{2}-$ and $\operatorname{Isom}(X)$-invariant metric which, as we will see later, also behaves nicely with respect to the $\mathbb{R}$-action. That is, the symmetric join of $X$ will become an example of a metric join. 


\subsection{Parametrizations of $\oplus X$}

Given a metric space $(X, d)$, first we want to put a metric on each line in $\oplus X$. We will do this by identifying each line with a subinterval of $\mathbb{R}$.

Define the functions $\langle\cdot, \cdot \mid \cdot, \cdot\rangle$ and $\langle\cdot \mid \cdot\rangle$. in $X$ as in 1.1 using the metric on $X$, and fix a basepoint $x_{0} \in X$. For every pair $(a, b) \in X^{2}$, denote

$$
\alpha:=-\left\langle b \mid x_{0}\right\rangle_{a} \quad \text { and } \quad \beta:=\left\langle a \mid x_{0}\right\rangle_{b},
$$

these are the end-point coordinates of the pair $(a, b)$ (with respect to $x_{0}$ ). Let $\llbracket a, b \rrbracket=\llbracket a, b \rrbracket_{x_{0}}$ be a copy of the interval $[\alpha, \beta] \subseteq \mathbb{R}$, and $\rrbracket a, b \llbracket$ its interior. Note that the interval $[\alpha, \beta] \subseteq \mathbb{R}$ always contains 0 and its length is

$$
\beta-\alpha=\left\langle a \mid x_{0}\right\rangle_{b}+\left\langle b \mid x_{0}\right\rangle_{a}=d(a, b) \geq 0 .
$$

It is convenient to think of $\llbracket a, b \rrbracket$ as a formal geodesic connecting $a$ to $b$; it indeed degenerates to a point when $a=b$. The convenience of this parametrization of $\llbracket a, b \rrbracket$ will be clear later when we define the $\operatorname{Isom}(X)$-action and the projection of $X$ to $\llbracket a, b \rrbracket$; the basepoint $x_{0}$ will project to $0 \in \llbracket a, b \rrbracket$, and $a$ and $b$ will project to the endpoints of $\llbracket a, b \rrbracket$. Also this parametrization will be needed to extend things to infinity when $X$ is a hyperbolic complex.

Define functions

$$
\begin{array}{ll} 
& \llbracket a, b ; \cdot \rrbracket=\llbracket a, b ; \cdot \rrbracket_{x_{0}}: \overline{\mathbb{R}} \rightarrow \llbracket a, b \rrbracket \quad \text { by } \quad \llbracket a, b ; t \rrbracket:=\theta[\alpha, \beta ; t] \\
\text { and } \quad \llbracket a, b ; \cdot \rrbracket^{\prime}=\llbracket a, b ; \cdot \rrbracket_{x_{0}}^{\prime}: \overline{\mathbb{R}} \rightarrow \llbracket a, b \rrbracket \quad \text { by } \quad \llbracket a, b ; t \rrbracket^{\prime}:=\theta^{\prime}[\alpha, \beta ; t],
\end{array}
$$

where $\theta$ and $\theta^{\prime}$ are the functions from 1.6 . By the definition of $\theta, \llbracket a, b ; \cdot \rrbracket$ is just the obvious extension of the identity map of $\llbracket a, b \rrbracket$, so it is a non-expanding surjection. A real number $t$ will be called appropriate for $\llbracket a, b \rrbracket$ if $t \in[\alpha, \beta]$. A number $t$ appropriate for $\llbracket a, b \rrbracket$ will be called the $x_{0}$-coordinate of the point $\llbracket a, b ; t \rrbracket$ in $\llbracket a, b \rrbracket$, or simply the coordinate when $x_{0}$ is understood. If $(\alpha, \beta)$ are the endpoint coordinates of $(a, b)$, then

$$
\llbracket a, b ; t \rrbracket^{\prime}=\llbracket a, b ; \theta^{\prime}[\alpha, \beta ; t] \rrbracket .
$$

This holds just because $\llbracket a, b ; \cdot \rrbracket$ is identity on appropriate numbers.

$\llbracket a, b ; \cdot \rrbracket^{\prime}$ is the smooth-out of $\llbracket a, b ; \cdot \rrbracket$. By Lemma 2 $\llbracket a, b ; \cdot \rrbracket^{\prime}$ is a non-expanding surjection, and it is an increasing homeomorphism when $a \neq b$. Denote

$$
\overleftrightarrow{x_{0}} X:=\bigsqcup\{\llbracket a, b \rrbracket \mid a, b \in X\}, \quad{\underset{x}{0}}_{0}^{\circledast} X:=\underset{x_{0}}{\diamond} X / \sim,
$$

where the equivalence relation $\sim$ identifies the left endpoint of each interval $\llbracket a, b \rrbracket$ with the point $\llbracket a, a \rrbracket$ and the right endpoint with $\llbracket b, b \rrbracket$. Thus we view $X$ 
as embedded in $\underset{x_{0}}{\otimes} X$ via $a \mapsto \llbracket a, a \rrbracket$, and abusing notations we view $\llbracket a, b \rrbracket$ as a subset both of $\underset{x_{0}}{\diamond}$ and of $\underset{x_{0}}{\circledast x}$.

We denote $|\cdot|$ the standard metric on each $\llbracket a, b \rrbracket$, that is $|x-y|$ is the distance between $x$ and $y$ in $\llbracket a, b \rrbracket$.

\subsection{The models $\otimes X$ and $\underset{x_{0}}{\oplus}$}

The above maps $\llbracket a, b ; \cdot \rrbracket^{\prime}$ induce surjections

$$
\llbracket \cdot, \cdot ; \rrbracket^{\prime}: X^{2} \times \overline{\mathbb{R}} \rightarrow{ }_{x_{0}}^{\diamond} X \quad \text { and } \quad \llbracket \cdot, \cdot ; \cdot \rrbracket^{\prime}: X^{2} \times \overline{\mathbb{R}} \rightarrow \underset{x_{0}^{\otimes}}{\otimes} X
$$

and passing to quotients, bijections

$$
\llbracket \cdot, \cdot ; \cdot \rrbracket^{\prime}: \diamond X \rightarrow \underset{x_{0}}{\diamond} \quad \text { and } \quad \llbracket \cdot, \cdot ; \cdot \rrbracket^{\prime}: \circledast X \rightarrow \underset{x_{0}^{\otimes}}{\otimes} X .
$$

To summarize:

- $\circledast X$ and ${ }_{x_{0}^{\oplus}}^{\circledast X}$ are two different models, or parametrizations, of the symmetric join;

- $\circledast X$ has a natural topology, and each line is topologically parameterized by $\overline{\mathbb{R}}$;

- ${ }_{x_{0}}^{\circledast} X$ is just a set, but lines in ${ }_{x_{0}}^{\circledast} X$ are metric spaces which are isometrically parameterized by closed subintervals of $\mathbb{R}$;

- the two models are identified by the bijection $\llbracket \cdot, \cdot ; \cdot \rrbracket^{\prime}$ in (10).

We induce the topology on $\underset{x}{\circledast x} X$ from $\otimes X$ by this bijection. Equivalently, the topology on $x_{0}^{\otimes} X$ is induced by the surjection $\llbracket \cdot, \cdot ; \cdot \rrbracket^{\prime}: X^{2} \times \overline{\mathbb{R}} \rightarrow{ }_{x_{0}^{\otimes}}^{\otimes}$. This topology is consistent with the metric topology on each line in $\underset{x_{0}^{\mathscr{P}}}{\stackrel{\otimes}{ }}$ because each $\llbracket a, b ; \cdot \rrbracket^{\prime}: \overline{\mathbb{R}} \rightarrow \llbracket a, b \rrbracket$ is a homeomorphism for $a \neq b$.

The bijection in (10) allows us to identify $\otimes X$ with $x_{0}^{\otimes} X$, so we will often use the simpler notation $\otimes X$ instead of $\underset{x_{0}}{\otimes} X$.

\subsection{The models $* X$ and $\underset{x_{0}}{*} X$}

There are similar models for the open symmetric join: denote

$$
{ }_{x_{0}^{*}}^{*} X:=\bigcup\left\{\rrbracket a, b \llbracket \subseteq \underset{x_{0}^{\circledast}}{\otimes} \mid(a, b) \in X^{2}\right\}=\left({ }_{x_{0}^{\circledast}}^{\circledast} X\right) \backslash X \subseteq{ }_{x_{0}^{\circledast}} X .
$$

Define the topology on $\underset{x_{0}^{*}}{*} X$ by its inclusion into $\underset{x_{0}}{\circledast 2}$.

Let $\Delta$ be the diagonal of $X^{2}$. We can assume $(a, b) \in X^{2} \backslash \Delta$ above, since $\rrbracket a, b \llbracket$ is empty otherwise. Let $\rrbracket a, b ; \cdot \llbracket^{\prime}: \mathbb{R} \rightarrow \rrbracket a, b \llbracket$ be the restriction of $\llbracket a, b ; \cdot \rrbracket^{\prime}$ 
to the interiors of the intervals; this restriction is a homeomorphism. In the quotient this gives bijections

$$
\rrbracket \cdot, \cdot ; \cdot \mathbb{I}^{\prime}:\left(X^{2} \backslash \Delta\right) \times \mathbb{R} \rightarrow \underset{x_{0}^{*} X}{*} \quad \text { and } \quad \rrbracket \cdot, \cdot ; \cdot \mathbb{\llbracket}^{\prime}: * X \rightarrow{ }_{x_{0}}^{*} X
$$

which induce the same topology on $x_{0}^{*} X$ as the one described above.

Our goal is to equip the symmetric join with a metric and three actions. In the process we will be using the two models interchangeably.

\subsection{Change of basepoint in $\oplus X$}

The parametrization $\llbracket a, b ; \cdot \rrbracket=\llbracket a, b ; \cdot \rrbracket_{x_{0}}$ of each line $\llbracket a, b \rrbracket=\llbracket a, b \rrbracket_{x_{0}}$ depends on the choice of $x_{0} \in X$, but the isometry type of $\llbracket a, b \rrbracket$ does not. Another choice of basepoint, $x_{1}$ gives another interval $\llbracket a, b \rrbracket_{x_{1}}:=\left[-\left\langle b \mid x_{1}\right\rangle_{a},\left\langle a \mid x_{1}\right\rangle_{b}\right]$ of length $d(a, b)$ and another identity map

$$
\llbracket a, b ; \cdot \rrbracket_{x_{1}}:\left[-\left\langle b \mid x_{1}\right\rangle_{a},\left\langle a \mid x_{1}\right\rangle_{b}\right] \rightarrow \llbracket a, b \rrbracket_{x_{1}} .
$$

We will always identify two such parametrizations by the unique isometry between $\llbracket a, b \rrbracket_{x_{0}}$ and $\llbracket a, b \rrbracket_{x_{1}}$ for all $a, b \in X$. Since the left endpoints $\llbracket a, b ;-\left\langle b \mid x_{0}\right\rangle_{a} \rrbracket_{x_{0}}$ and $\llbracket a, b ;-\left\langle b \mid x_{1}\right\rangle_{a} \rrbracket_{x_{1}}$ must be identified, the explicit formula is

$$
\llbracket a, b ; t \rrbracket_{x_{1}}=\llbracket a, b ; t+\left\langle b \mid x_{1}\right\rangle_{a}-\left\langle b \mid x_{0}\right\rangle_{a} \rrbracket_{x_{0}}=\llbracket a, b ; t+\left\langle a, b \mid x_{1}, x_{0}\right\rangle \rrbracket_{x_{0}}, \quad t \in \overline{\mathbb{R}} .
$$

\subsection{The $\mathbb{R}$-action on $\oplus X$}

Let $X$ be an arbitrary metric space. The shift action

$$
+: \mathbb{R} \times \overline{\mathbb{R}} \rightarrow \overline{\mathbb{R}}, \quad(r, t) \mapsto r^{+} t, \quad \text { where } \quad r^{+} t:=r+t
$$

with the convention $r \pm \infty= \pm \infty$, induces the obvious $\mathbb{R}$-action on $X^{2} \times \overline{\mathbb{R}}$ by translations in the $\overline{\mathbb{R}}$-coordinate, and, passing to the quotient, the $\mathbb{R}$-action on $\otimes X$ :

$$
+: \mathbb{R} \times(\otimes X) \rightarrow \circledast X, \quad(r, z) \mapsto r^{+} z .
$$

This action preserves lines and fixes $X$ pointwise. The $\mathbb{R}$-orbits in $\otimes X$ are exactly the points of $X$ and the interiors of the lines in $\otimes X$. The bijection $\llbracket \cdot, \cdot \cdot \rrbracket^{\prime}$ transfers this further to the action

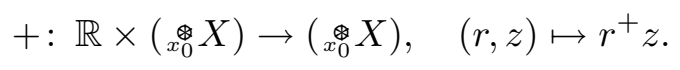

The explicit formula for the action is $r^{+} \llbracket a, b ; t \rrbracket^{\prime}:=\llbracket a, b ; r+t \rrbracket^{\prime}$. 
Lemma 5 For each $x \in \llbracket a, b \rrbracket \subseteq \underset{x_{0}^{\otimes} X}{\otimes}$, the $\mathbb{R}$-orbit map $\mathbb{R} \rightarrow(\llbracket a, b \rrbracket,|\cdot|)$, $r \mapsto r^{+} x$, is non-expanding, therefore continuous. In addition, if $x \in \rrbracket a, b \llbracket$, then the orbit map is an increasing homeomorphism of $\mathbb{R}$ onto $\rrbracket a, b \llbracket$.

Proof (a) $x=\llbracket a, b ; t \rrbracket^{\prime}$ for some $t \in \overline{\mathbb{R}}$. Since $\llbracket a, b ; \cdot \rrbracket^{\prime}$ is non-expanding, $\left|r_{1}^{+} x-r_{2}^{+} x\right|=\left|\llbracket a, b ; r_{1}+t \rrbracket^{\prime}-\llbracket a, b ; r_{1}+t \rrbracket^{\prime}\right| \leq\left|\left(r_{1}+t\right)-\left(r_{2}+t\right)\right|=\left|r_{1}-r_{2}\right|$, i.e. the orbit map is non-expanding. If $x \in \rrbracket a, b \llbracket$ then $a \neq b, t \in \mathbb{R}$ and the orbit map $r \mapsto \rrbracket a, b ; r+t \llbracket^{\prime}$ is an increasing homeomorphism $\mathbb{R} \rightarrow \rrbracket a, b \llbracket$ because it is the composition of increasing homeomorphisms $\mathbb{R} \rightarrow \mathbb{R} \rightarrow \rrbracket a, b \llbracket$, $r \mapsto r+t \mapsto \rrbracket a, b ; r+t \llbracket^{\prime}$.

\subsection{The $\mathbb{Z}_{2}$-action on $\otimes X$}

The map $\star: \diamond X \rightarrow \diamond X$ given by $\llbracket a, b ; t \rrbracket^{\star}:=\llbracket b, a ;-t \rrbracket$ for $a, b \in X$ and an appropriate $t$ is a well-defined involution of $\diamond X$. Note that $\llbracket a, a \rrbracket^{\star}=\llbracket a, a \rrbracket$. Also if $\llbracket a, b ; t \rrbracket$ is the left endpoint of $\llbracket a, b \rrbracket$ (which is identified with $\llbracket a, a \rrbracket$ in $\circledast X$ ) then necessarily $t=-\left\langle b \mid x_{0}\right\rangle_{a}$, so $\llbracket a, b ; t \rrbracket^{\star}=\llbracket b, a ;\left\langle b \mid x_{0}\right\rangle_{a} \rrbracket$ is the right endpoint of $\llbracket b, a \rrbracket$ (which is also identified with $\llbracket a, a \rrbracket$ ). Therefore the same formula gives an involution $\star: \otimes X \rightarrow \otimes X$ in the quotient, and $\star$ fixes $X$ pointwise.

\subsection{The $\operatorname{Isom}(X)$-action on $\otimes X$}

$\operatorname{Isom}(X)$ acts on $\underset{x_{0}}{\diamond} X$ by

$$
\begin{aligned}
& g \llbracket a, b ; t \rrbracket:=\llbracket g a, g b ; t+\left\langle a, b \mid x_{0}, g^{-1} x_{0}\right\rangle \rrbracket, \\
& g \in \operatorname{Isom}(X), \quad(a, b) \in X^{2}, \quad t \in\left[-\left\langle b \mid x_{0}\right\rangle_{a},\left\langle a \mid x_{0}\right\rangle_{b}\right] .
\end{aligned}
$$

One checks that if $t$ runs through $\left[-\left\langle b \mid x_{0}\right\rangle_{a},\left\langle a \mid x_{0}\right\rangle_{b}\right]$ then $t+\left\langle a, b \mid x_{0}, g^{-1} x_{0}\right\rangle$ runs through $\left[-\left\langle g b \mid x_{0}\right\rangle_{g a},\left\langle g a \mid x_{0}\right\rangle_{g b}\right]$, so $g$ maps each line $\llbracket a, b \rrbracket$ isometrically onto the line $\llbracket g a, g b \rrbracket$.

This is indeed an action since for id $\in \operatorname{Isom}(X)$,

$$
\text { id } \llbracket a, b ; t \rrbracket=\llbracket \operatorname{id} a, \operatorname{id} b ; t+\left\langle a, b \mid x_{0}, \mathrm{id} x_{0}\right\rangle \rrbracket=\llbracket a, b ; t \rrbracket
$$

and for $f, g \in \operatorname{Isom}(X)$,

$$
\begin{aligned}
& f(g \llbracket a, b ; t \rrbracket)=f \llbracket g a, g b ; t+\left\langle a, b \mid x_{0}, g^{-1} x_{0}\right\rangle \rrbracket \\
& =\llbracket f g a, f g b ; t+\left\langle a, b \mid x_{0}, g^{-1} x_{0}\right\rangle+\left\langle g a, g b \mid x_{0}, f^{-1} x_{0}\right\rangle \rrbracket \\
& =\llbracket f g a, f g b ; t+\left\langle a, b \mid x_{0}, g^{-1} x_{0}\right\rangle+\left\langle a, b \mid g^{-1} x_{0}, g^{-1} f^{-1} x_{0}\right\rangle \rrbracket \\
& =\llbracket f g a, f g b ; t+\left\langle a, b \mid x_{0}, g^{-1} f^{-1} x_{0}\right\rangle \rrbracket \\
& =\llbracket f g a, f g b ; t+\left\langle a, b \mid x_{0},(f g)^{-1} x_{0}\right\rangle \rrbracket=(f g) \llbracket a, b ; t \rrbracket .
\end{aligned}
$$


Since $g$ sends the left endpoint of $\llbracket a, b \rrbracket$ to the left endpoint of $\llbracket g a, g b \rrbracket$, and similarly for the right endpoints, the above action descends to an action on ${ }_{x_{0}^{\oplus}} X$, given by the same formula (14), and therefore to an action on $\otimes X$.

Lemma 6 Let $X$ be an arbitrary metric space.

(a) The $\operatorname{Isom}(X)$ - and $\mathbb{R}$-actions on $\oplus X$ commute.

(b) The $\operatorname{Isom}(X)$ - and $\mathbb{Z}_{2}$-actions on $\oplus X$ commute.

(c) The $\mathbb{Z}_{2}$-action anticommutes with the $\mathbb{R}$-action on $\otimes X$ : $\left(r^{+} x\right)^{\star}=(-r)^{+} x^{\star}$ for $x \in \otimes X$ and $r \in \mathbb{R}$.

(d) All the three actions on $\otimes X$ map lines onto lines and are independent of $x_{0}$. The $\mathbb{Z}_{2}$ - and $\mathbb{R}$-actions fix $X$ pointwise.

(e) The $\operatorname{Isom}(X)$-action on $\otimes X$ is an extension of the $\operatorname{Isom}(X)$-action on $X$.

Proof (a) The difficulty is that the $\operatorname{Isom}(X)$ - and $\mathbb{R}$-actions are defined with respect to different parametrizations, $\llbracket \cdot, \cdot ; \cdot \rrbracket$ and $\llbracket \cdot, \cdot ; \cdot \rrbracket^{\prime}$, respectively. But the relation (7) between the two parametrizations and the shift invariance of $\theta^{\prime}$ will suffice for the proof. Pick any point $\llbracket a, b ; t \rrbracket^{\prime} \in \otimes X$ and $g \in \operatorname{Isom}(X)$ and denote $s:=\left\langle a, b \mid x_{0}, g^{-1} x_{0}\right\rangle$. By direct calculation, if $(\alpha, \beta)$ are the endpoint coordinates of $(a, b)$, then $(\alpha+s, \beta+s)$ are the endpoint coordinates of $(g a, g b)$. Hence for $r \in \mathbb{R}$ and $g \in \operatorname{Isom}(X)$,

$$
\begin{aligned}
& r^{+}\left(g \llbracket a, b ; t \rrbracket^{\prime}\right)=r^{+}\left(g \llbracket a, b ; \theta^{\prime}[\alpha, \beta ; t] \rrbracket\right)=r^{+} \llbracket g a, g b ; \theta^{\prime}[\alpha, \beta ; t]+s \rrbracket \\
& =r^{+} \llbracket g a, g b ; \theta^{\prime}[\alpha+s, \beta+s ; t+s] \rrbracket=r^{+} \llbracket g a, g b ; t+s \rrbracket^{\prime}=\llbracket g a, g b ; r+t+s \rrbracket^{\prime} \\
& =\llbracket g a, g b ; \theta^{\prime}\left[\alpha+s, \beta+s ; r+t+s \rrbracket \rrbracket=\llbracket g a, g b ; \theta^{\prime}[\alpha, \beta ; r+t]+s \rrbracket\right. \\
& =g \llbracket a, b ; \theta^{\prime}[\alpha, \beta ; r+t] \rrbracket=g \llbracket a, b ; r+t \rrbracket^{\prime}=g\left(r^{+} \llbracket a, b ; t \rrbracket^{\prime}\right) .
\end{aligned}
$$

(d) The involution $\star$ is independent of $x_{0}$ because for another $x_{1} \in X$, by the change of basepoint formula (13),

$$
\begin{aligned}
& \llbracket a, b ; r \rrbracket_{x_{1}}^{\star}=\llbracket a, b ; r+\left\langle a, b \mid x_{1}, x_{0}\right\rangle \rrbracket_{x_{0}}^{\star}=\llbracket b, a ;-r-\left\langle a, b \mid x_{1}, x_{0}\right\rangle \rrbracket_{x_{0}} \\
& =\llbracket b, a ;-r-\left\langle a, b \mid x_{1}, x_{0}\right\rangle+\left\langle b, a \mid x_{0}, x_{1}\right\rangle \rrbracket_{x_{1}}=\llbracket b, a ;-r \rrbracket_{x_{1}} .
\end{aligned}
$$

Similarly, one checks using (13) and the $\operatorname{Isom}(X)$-invariance of the double difference that

$$
g \llbracket a, b ; r \rrbracket_{x_{1}}=\llbracket g a, g b ; r+\left\langle a, b \mid x_{1}, g^{-1} x_{1}\right\rangle \rrbracket_{x_{1}},
$$

i.e. the $\operatorname{Isom}(X)$-action does not depend on the choice of $x_{0}$. For the $\mathbb{R}$-action, use (13) and the shift invariance of $\theta^{\prime}$ to prove $r^{+} \llbracket a, b ; t \rrbracket_{x_{1}}=\llbracket a, b ; r+t \rrbracket_{x_{1}}$.

(b), (c), (e) and the rest of (d) directly follow from definitions. 


\subsection{Projecting $X$ to lines in $\circledast X$}

Our metric join construction will work for an arbitrary metric space, but for inspiration consider first the classical hyperbolic space $\mathbb{H}^{n}$, or more generally, a CAT(-1)-space. Let $a, a^{\prime}, b, b^{\prime} \in \partial \mathbb{H}^{n}$ and denote $\left[b, b^{\prime} \mid a\right]$ the nearest-point projection of $a$ to the geodesic from $b$ to $b^{\prime}$. Then the double difference $\left\langle a, a^{\prime} \mid b^{\prime}, b\right\rangle$ indeed makes sense when $a, a^{\prime}, b, b^{\prime}$ are in the boundary of $\mathbb{H}^{n}$, and it equals the directed distance from $\left[b, b^{\prime} \mid a\right]$ to $\left[b, b^{\prime} \mid a^{\prime}\right]$ along the geodesic from $b$ to $b^{\prime}$, or, symmetrically, the directed distance from $\left[a, a^{\prime} \mid b\right]$ to $\left[a, a^{\prime} \mid b^{\prime}\right]$ along the geodesic from $a$ to $a^{\prime}$ (see for example [3, 1.3]). We will use this observation in the constructions that follow (though the endpoints of geodesics will be in the metric space rather than in the ideal boundary).

Again we fix a basepoint $x_{0} \in X$.

Definition 7 Let $a, a^{\prime}, b \in X$. The coordinate of the projection of $b \in X$ to the line $\llbracket a, a^{\prime} \rrbracket \subseteq{ }_{x_{0}^{\otimes}}^{\otimes} X$ is $\left\langle a, a^{\prime} \mid b\right\rangle:=\left\langle a, a^{\prime} \mid b, x_{0}\right\rangle$ and the projection of $b$ to the line $\llbracket a, a^{\prime} \rrbracket$ is $\llbracket a, a^{\prime} \mid b \rrbracket:=\llbracket a, a^{\prime} ;\left\langle a, a^{\prime} \mid b\right\rangle \rrbracket$.

Since $\llbracket a, a^{\prime} \rrbracket$ is identified with an interval in $\mathbb{R},\left\langle a, a^{\prime} \mid b\right\rangle$ is essentially the same thing as $\llbracket a, a^{\prime} \mid b \rrbracket$. We only use two different notations to emphasize that $\left\langle a, a^{\prime} \mid b\right\rangle$ means a real number and $\llbracket a, a^{\prime} \mid b \rrbracket$ represents a point in the metric space $\llbracket a, a^{\prime} \rrbracket$.

The parametrization $\llbracket a, a^{\prime} ; \cdot \rrbracket$ and the above definitions are chosen so that in particular,

$$
\llbracket a, a^{\prime} \mid a \rrbracket=\llbracket a, a^{\prime} ;\left\langle a, a^{\prime} \mid a\right\rangle \rrbracket=\llbracket a, a^{\prime} ;\left\langle a, a^{\prime} \mid a, x_{0}\right\rangle \rrbracket=\llbracket a, a^{\prime} ;-\left\langle a^{\prime} \mid x_{0}\right\rangle_{a} \rrbracket=a
$$

(recall that we do not distinguish between $a \in X$ and the left endpoint of $\left.\llbracket a, a^{\prime} \rrbracket \subseteq{ }_{x_{0}}^{\otimes} X\right)$ and similarly $\llbracket a, a^{\prime} \mid a^{\prime} \rrbracket=a^{\prime}$, that is $a$ and $a^{\prime}$ project to themselves. Note also that $\llbracket a, a^{\prime} \mid x_{0} \rrbracket=\llbracket a, a^{\prime} ;\left\langle a, a^{\prime} \mid x_{0}\right\rangle \rrbracket=\llbracket a, a^{\prime} ; 0 \rrbracket ;$ in other words, the basepoint $x_{0}$ always project to the "origin" $\llbracket a, a^{\prime} ; 0 \rrbracket$ of $\llbracket a, a^{\prime} \rrbracket$.

The following lemma gives an equivalent description of the projection.

Lemma 8 For $a, a^{\prime}, b \in X, \llbracket a, a^{\prime} \mid b \rrbracket$ is the unique point $c$ in $\llbracket a, a^{\prime} \rrbracket$ that satisfies

$$
|a-c|-\left|c-a^{\prime}\right|=d(a, b)-d\left(b, a^{\prime}\right) .
$$

\section{Proof}

$$
\begin{aligned}
& \mid a- \llbracket a, a^{\prime}|b \rrbracket|=\left|\llbracket a, a^{\prime}\right| a \rrbracket-\llbracket a, a^{\prime}|b \rrbracket| \\
& \quad=\left|\left\langle a, a^{\prime} \mid a\right\rangle-\left\langle a, a^{\prime} \mid b\right\rangle\right|=\left|\left\langle a, a^{\prime} \mid a, b\right\rangle\right|=\left\langle a^{\prime} \mid b\right\rangle_{a}
\end{aligned}
$$


and similarly, $\left|\llbracket a, a^{\prime}\right| b \rrbracket-a^{\prime} \mid=\langle a \mid b\rangle_{a^{\prime}}$, hence

$$
\left|a-\llbracket a, a^{\prime}\right| b \rrbracket|-| \llbracket a, a^{\prime}\left|b \rrbracket-a^{\prime}\right|=\left\langle a^{\prime} \mid b\right\rangle_{a}+\langle a \mid b\rangle_{a^{\prime}}=d(a, b)-d\left(a^{\prime}, b\right) .
$$

For any $a, a^{\prime}, b, b^{\prime} \in X$,

$$
\left\langle a, a^{\prime} \mid b^{\prime}\right\rangle-\left\langle a, a^{\prime} \mid b\right\rangle=\left\langle a, a^{\prime} \mid b^{\prime}, x_{0}\right\rangle-\left\langle a, a^{\prime} \mid b, x_{0}\right\rangle=\left\langle a, a^{\prime} \mid b^{\prime}, b\right\rangle,
$$

i.e. the meaning of $\left\langle a, a^{\prime} \mid b^{\prime}, b\right\rangle$ now is the difference of the coordinates of the projections of $b^{\prime}$ and $b$ to $\llbracket a, a^{\prime} \rrbracket$.

Lemma 9 (a) The projection map $\llbracket \cdot, \cdot \cdot \rrbracket$ is independent of the choice of basepoint $x_{0}$.

(b) $\llbracket \cdot, \cdot \cdot \cdot \rrbracket$ is $\operatorname{Isom}(X)$-invariant, i.e.

$$
g \llbracket a, a^{\prime}\left|b \rrbracket=\llbracket g a, g a^{\prime}\right| g b \rrbracket \quad \text { for } a, a^{\prime}, b \in X, \quad g \in \operatorname{Isom}(\mathrm{X}) .
$$

(c) The projection map relates to the $\mathbb{Z}_{2}$-action on $\otimes X$ by the formula

$$
\llbracket a, a^{\prime}\left|b \rrbracket^{\star}=\llbracket a^{\prime}, a\right| b \rrbracket, \quad a, a^{\prime}, b \in X .
$$

Proof (a) and (b) follow from Lemma 8 , and (c) follows from definitions:

$$
\llbracket a, a^{\prime}\left|b \rrbracket^{\star}=\llbracket a, a^{\prime}\right|\left\langle a, a^{\prime} \mid b\right\rangle \rrbracket^{\star}=\llbracket a^{\prime}, a\left|-\left\langle a, a^{\prime} \mid b\right\rangle \rrbracket=\llbracket a^{\prime}, a\right|\left\langle a^{\prime}, a \mid b\right\rangle \rrbracket=\llbracket a^{\prime}, a \mid b \rrbracket .
$$

\subsection{Projection and change of basepoint}

The change of basepoint formula (13) impies that

$$
\llbracket a, b ; 0 \rrbracket_{x_{1}}=\llbracket a, b ;\left\langle a, b \mid x_{1}\right\rangle \rrbracket=\llbracket a, b \mid x_{1} \rrbracket .
$$

In other words, $\llbracket a, b ; \cdot \rrbracket_{x_{1}}$ is the isometric orientation-preserving reparametrization of $\llbracket a, b \rrbracket$ whose origin $\llbracket a, b ; 0 \rrbracket_{x_{1}}$ is the projection of $x_{1}$ to $\llbracket a, b \rrbracket$.

\section{$3 \quad$ The metric $d_{*}$ on $\otimes X$}

\subsection{The cocycle $\beta^{\times}$in $\circledast X$}

Definition 10 Let $X$ be any metric space. For $u \in X$ and $x=\llbracket a, a^{\prime} ; s \rrbracket \in \otimes X$, where $a, a^{\prime} \in X$ and $s$ is appropriate, let $\ell(u, x):=\left\langle a \mid a^{\prime}\right\rangle_{u}+\left|s-\left\langle a, a^{\prime} \mid u\right\rangle\right|$ (see Figure 1).

For $u \in X, x, y \in \otimes X$, let $\beta_{u}^{\times}(x, y):=\ell(u, x)-\ell(u, y)$. 
This gives a function $\beta^{\times}: X \times(\otimes X)^{2} \rightarrow \mathbb{R}$ of three variables $(u, x, y)$. The definition of $\ell$ mimics the case of a tree: if the imaginary triangle $\left\{u, a, a^{\prime}\right\}$ was degenerate to a tripod, then $\ell(u, x)$ would be exactly the distance between $u$ and $x$. Note also that when $x \in X, \ell(u, x)=d(u, x)$, so the restriction of $\beta^{\times}$ to $X \times X^{2}$ is the distance cocycle: $\beta_{u}^{\times}(x, y)=d(u, x)-d(u, y)$.

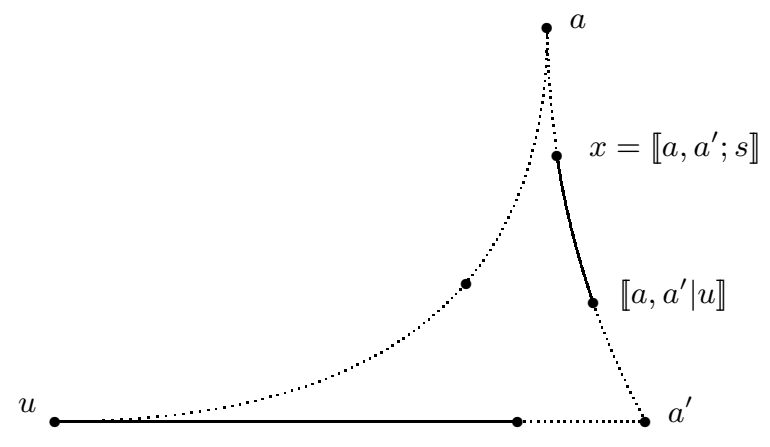

Figure 1: $\ell(u, x)$

\section{Theorem 11}

(a) The above functions $\ell: X \times(\otimes X) \rightarrow[0, \infty)$ and $\beta^{\times}: X \times(\oplus X)^{2} \rightarrow \mathbb{R}$ are well-defined, independent of $x_{0}$, and Lipschitz in the first variable $u \in X$ for each fixed $(x, y)$.

(b) $\beta_{u}^{\times}$satisfies the cocycle condition $\beta_{u}^{\times}(x, y)+\beta_{u}^{\times}(y, z)=\beta_{u}^{\times}(x, z)$.

(c) $\beta_{u}^{\times}$is $\mathbb{Z}_{2}$-invariant in each variable: $\beta_{u}^{\times}(x, y)=\beta_{u}^{\times}\left(x^{\star}, y\right)=\beta_{u}^{\times}\left(x, y^{\star}\right)$.

(d) $\beta^{\times}$is $\operatorname{Isom}(X)$-invariant: $\beta_{g u}^{\times}(g x, g y)=\beta_{u}^{\times}(x, y)$ for $g \in \operatorname{Isom}(X)$.

Proof (a) It suffices to show (a) for $\ell$. Recall from section 2 that $\otimes X$ is a quotient of $\diamond X$, that is each $x \in X \subseteq \otimes X$ can be represented as $\llbracket a, a^{\prime} ; s \rrbracket$ in many ways. It is to be shown that the formula for $\ell$ does not depend on the representations of $x \in \circledast X$.

Assume $\llbracket a, x ; s \rrbracket$ is the right endpoint of $\llbracket a, x \rrbracket \subseteq \diamond X$ and $s$ is appropriate,

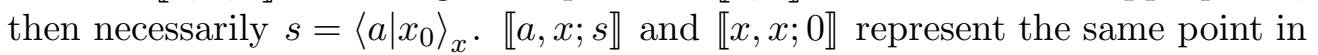
$X \subseteq \otimes X$.

$$
\begin{aligned}
& \ell(u, \llbracket a, x ; s \rrbracket)=\langle a \mid x\rangle_{u}+\left|\left\langle a \mid x_{0}\right\rangle_{x}-\langle a, x \mid u\rangle\right| \\
& =\langle a \mid x\rangle_{u}+\left|\left\langle a, x \mid x, x_{0}\right\rangle-\left\langle a, x \mid u, x_{0}\right\rangle\right|=\langle a \mid x\rangle_{u}+|\langle a, x \mid x, u\rangle| \\
& =\langle a \mid x\rangle_{u}+\langle a \mid u\rangle_{x}=d(x, u)=\langle x \mid x\rangle_{u}+|0-\langle x, x \mid u\rangle|=\ell(u, \llbracket x, x ; 0 \rrbracket),
\end{aligned}
$$


so $\ell$ is well-defined on the level of the quotient $\otimes X$.

$s$ is the $x_{0}$-coordinate of $x$ in $\llbracket a, a^{\prime} \rrbracket$, then by (13) for another basepoint $x_{1}$, the $x_{1}$-coordinate of $x$ is $s+\left\langle a, a^{\prime} \mid x_{0}, x_{1}\right\rangle$. The identity

$$
\left|s-\left\langle a, a^{\prime} \mid u, x_{0}\right\rangle\right|=\left|\left(s+\left\langle a, a^{\prime} \mid x_{0}, x_{1}\right\rangle\right)-\left\langle a, a^{\prime} \mid u, x_{1}\right\rangle\right|
$$

shows that $\ell$ is independent of $x_{0}$.

By Lemma 8, $\ell(u, x)$ is uniquely determined by the distances between points $u, a, a^{\prime}, \llbracket a, a^{\prime} \mid u \rrbracket$ and $x$. These distances are preserved under isometries of $X$, hence $\ell$ is $\operatorname{Isom}(X)$-invariant.

By the triangle inequality,

$$
\begin{aligned}
& |\ell(u, x)-\ell(v, x)|=\left|\left\langle a \mid a^{\prime}\right\rangle_{u}-\left\langle a \mid a^{\prime}\right\rangle_{v}\right|+|| s-\left\langle a, a^{\prime} \mid u\right\rangle|-| s-\left\langle a, a^{\prime} \mid v\right\rangle|| \\
& \leq d(u, v)+\left|\left\langle a, a^{\prime} \mid v\right\rangle-\left\langle a, a^{\prime} \mid u\right\rangle\right|=d(u, v)+\left|\left\langle a, a^{\prime} \mid v, u\right\rangle\right| \leq 2 d(u, v),
\end{aligned}
$$

so $\ell(u, x)$ is Lipschitz in the variable $u$.

Parts (b) and (c) of the lemma are straightforward from definitions, (d) follows from definitions and the change of basepoint formula (13).

Lemma 12 Let $u, a, a^{\prime} \in X, x=\llbracket a, a^{\prime} ; s \rrbracket, y=\llbracket a, a^{\prime} ; t \rrbracket$ with appropriate $s$ and $t$, then

(a) $\left|\beta_{u}^{\times}(x, y)\right| \leq|s-t| \leq d\left(a, a^{\prime}\right), \quad \beta_{a}^{\times}(x, y)=s-t$,

(b) $\left|\beta_{u}^{\times}\left(a, a^{\prime}\right)\right| \leq d\left(a, a^{\prime}\right), \quad \beta_{a}^{\times}\left(a, a^{\prime}\right)=-d\left(a, a^{\prime}\right)$.

Proof From the definition of $\beta^{\times}$,

$$
\begin{gathered}
\beta_{u}^{\times}(x, y)=\left|s-\left\langle a, a^{\prime} \mid u\right\rangle\right|-\left|t-\left\langle a, a^{\prime} \mid u\right\rangle\right| \leq|s-t| \leq d\left(a, a^{\prime}\right), \\
\beta_{a}^{\times}(x, y)=\left|s-\left\langle a, a^{\prime} \mid a\right\rangle\right|-\left|t-\left\langle a, a^{\prime} \mid a\right\rangle\right| \\
\quad=\left(s-\left\langle a^{\prime} \mid x_{0}\right\rangle_{a}\right)-\left(t-\left\langle a^{\prime} \mid x_{0}\right\rangle_{a}\right)=s-t .
\end{gathered}
$$

If $x=a$ and $y=a^{\prime}$, then $s=-\left\langle a^{\prime} \mid x_{0}\right\rangle_{a}$ and $t=\left\langle a \mid x_{0}\right\rangle_{a^{\prime}}$, so

$$
\begin{aligned}
& \beta_{u}^{\times}\left(a, a^{\prime}\right) \leq|s-t|=\left|-\left\langle a^{\prime} \mid x_{0}\right\rangle_{a}-\left\langle a \mid x_{0}\right\rangle_{a^{\prime}}\right|=d\left(a, a^{\prime}\right), \\
& \beta_{a}^{\times}\left(a, a^{\prime}\right)=s-t=-\left\langle a^{\prime} \mid x_{0}\right\rangle_{a}-\left\langle a \mid x_{0}\right\rangle_{a^{\prime}}=-d\left(a, a^{\prime}\right) .
\end{aligned}
$$




\subsection{The pseudometric $d^{\times}$in $\oplus X$}

For $x, y \in \otimes X$ define

$$
d^{\times}(x, y):=\sup _{u \in X}\left|\beta_{u}^{\times}(x, y)\right|
$$

\section{Theorem 13}

(a) The function $d^{\times}$above is a well-defined $\operatorname{Isom}(X)$-invariant pseudometric on $\circledast X$ independent of $x_{0}$.

(b) The inclusion of each line $(\llbracket a, b \rrbracket,|\cdot|) \hookrightarrow\left(\circledast X, d^{\times}\right), a, b \in X$, is an isometric embedding.

(c) The canonical embedding $(X, d) \hookrightarrow\left(\circledast X, d^{\times}\right)$is an isometric embedding.

Proof (a) Let $x=\llbracket a, a^{\prime} ; s \rrbracket, y=\llbracket b, b^{\prime} ; t \rrbracket$ for appropriate $s$ and $t$. By the cocycle condition and Lemma 12 .

$$
\left|\beta_{u}^{\times}(x, y)\right| \leq\left|\beta_{u}^{\times}(x, a)\right|+\left|\beta_{u}^{\times}(a, b)\right|+\left|\beta_{u}^{\times}(b, y)\right| \leq d\left(a, a^{\prime}\right)+d(a, b)+d\left(b, b^{\prime}\right)
$$

for all $u \in X$, so $d^{\times}(x, y) \in[0, \infty)$.

The product $\left\langle a \mid a^{\prime}\right\rangle_{u}$ is independent of $x_{0}$ because it is expressed in terms of the metric $d$, the projection $\llbracket a, a^{\prime} \mid u \rrbracket$ is independent by Lemma 8 . The quantity $\left|s-\left\langle a, a^{\prime} \mid u\right\rangle\right|$ is the distance between $x$ and $\llbracket a, a^{\prime} \mid u \rrbracket$ in $\llbracket a, a^{\prime} \rrbracket$, so it is independent of $x_{0}$, and similarly for all the terms in the definition of $\beta^{\times}$, so this shows the independence of $\beta^{\times}$. The same argument provides $\operatorname{Isom}(X)$-invariance of $d^{\times}$.

Pick any triple $x, y, z \in \oplus X$ and any $\varepsilon>0$. By the definition of $d^{\times}$, there is $u \in X$ such that $d^{\times}(x, z)-\varepsilon \leq\left|\beta_{u}^{\times}(x, z)\right|$, therefore

$$
d^{\times}(x, z) \leq\left|\beta_{u}^{\times}(x, z)\right|+\varepsilon \leq\left|\beta_{u}^{\times}(x, y)\right|+\left|\beta_{u}^{\times}(y, z)\right|+\varepsilon \leq d^{\times}(x, y)+d^{\times}(y, z)+\varepsilon .
$$

Since this holds for each $\varepsilon>0$, the triangle inequality for $d^{\times}$follows. Since $\beta_{u}^{\times}(x, x)=0$ for all $u \in X$, then $d^{\times}(x, x)=0$, so $d^{\times}$is a pseudometric.

(b) If $x$ and $y$ lie on the same line $\llbracket a, a^{\prime} \rrbracket$ in ${ }_{x_{0}^{\circledR}}^{\circledR} X$, then $x=\llbracket a, a^{\prime} ; s \rrbracket$ and $y=\llbracket b, b^{\prime} ; t \rrbracket$ for some appropriate $s$ and $t$. By Lemma 12(a), $d^{\times}(x, y)=|s-t|$.

(c) If $a, b \in X$, then by part (b), $d^{\times}(a, b)$ equals the length of the interval $\llbracket a, b \rrbracket$. But $\llbracket a, b \rrbracket$ was chosen so that its length is $d(a, b)$. 


\subsection{The metric $d_{*}=\circledast d$ on $\circledast X$}

Define a function $\otimes d:(\circledast X)^{2} \rightarrow[0, \infty)$ by

$$
\otimes d(x, y):=\int_{-\infty}^{\infty} d^{\times}\left(r^{+} x, r^{+} y\right) \frac{e^{-|r|}}{2} d r, \quad x, y \in \otimes X,
$$

where $r^{+}$comes from the $\mathbb{R}$-action on $\otimes X$ described in 2.6. For simplicity we will use the notation $d_{*}$ instead of $\oplus d$.

Theorem 14 The function $d_{*}$ above is a well-defined $\operatorname{Isom}(X)$-invariant metric on $\otimes X$ independent of the choice of $x_{0}$.

Proof By Theorem 13(b), $d^{\times}$induces the original topology on each line, therefore by the triangle inequality for $d^{\times}$, the restriction of $d^{\times}$to each product $\llbracket a, a^{\prime} \rrbracket \times \llbracket b, b^{\prime} \rrbracket$ is continuous. By Lemma 5 for fixed $x$ and $y, d^{\times}\left(r^{+} x, r^{+} y\right)$ is continuous in $r$. First assume $x, y \in * X$. By Lemma [5] and Theorem [13(b), the $\mathbb{R}$-orbit maps $\mathbb{R} \rightarrow\left(\otimes X, d^{\times}\right)$are non-expanding, hence

$$
0 \leq d^{\times}\left(r^{+} x, r^{+} y\right) \leq d^{\times}\left(r^{+} x, x\right)+d^{\times}(x, y)+d^{\times}\left(y, r^{+} y\right) \leq d^{\times}(x, y)+2|r|
$$

for all $r \in \mathbb{R}$. If $x \in X$, then $d^{\times}\left(r^{+} x, x\right)=d^{\times}(x, x)=0$, and the same inequality as above holds, and similarly for $y \in X$. This inequality implies that $d_{*}(x, y)$ is a well-defined number in $[0, \infty)$ for all $x, y \in \oplus X$. The triangle inequality, the $\operatorname{Isom}(X)$-invariance and independence of $x_{0}$ follow from those of $d^{\times}$. Also, $d_{*}(x, x)=0$.

It remains to show that $x \neq y$ implies $d_{*}(x, y)>0$. Pick any $x \in \llbracket a, a^{\prime} \rrbracket$ and $y \in \llbracket b, b^{\prime} \rrbracket$ with $x \neq y$. If $a \neq b$, then, by Theorem 13(c), $d^{\times}(a, b)>0$. Since $r^{+} x \rightarrow a$ and $r^{+} y \rightarrow b$ as $r \rightarrow-\infty$, there exists $r_{0} \in \mathbb{R}$ such that for all $r \leq r_{0}$,

$$
d^{\times}\left(r^{+} x, a\right) \leq d(a, b) / 3 \quad \text { and } \quad d^{\times}\left(r^{+} y, b\right) \leq d(a, b) / 3,
$$

hence $d^{\times}\left(r^{+} x, r^{+} y\right) \geq d^{\times}(a, b) / 3$ and

$$
d_{*}(x, y) \geq \int_{-\infty}^{r_{0}} d^{\times}\left(r^{+} x, r^{+} y\right) \frac{e^{-|r|}}{2} d r \geq \int_{-\infty}^{r_{0}} d^{\times}(a, b) \frac{e^{-|r|}}{6} d r>0 .
$$

The case $a^{\prime} \neq b^{\prime}$ is similar, so now we can assume that $a=b$ and $a^{\prime}=b^{\prime}$, i.e. $x$ and $y$ lie on the same line $\llbracket a, a^{\prime} \rrbracket$, and $x \neq y$. For all $r \in \mathbb{R}, r^{+} x$ and $x^{+} y$ lie on the same line $\llbracket a, a^{\prime} \rrbracket$ and $r^{+} x \neq r^{+} y$; then by Theorem 13(b), $d^{\times}\left(r^{+} x, r^{+} y\right)>0$. Since $d^{\times}\left(r^{+} x, r^{+} y\right)$ is continuous in $r$,

$$
d_{*}(x, y)=\int_{-\infty}^{\infty} d^{\times}\left(r^{+} x, r^{+} y\right) \frac{e^{-|r|}}{2} d r>0 .
$$


Remark (16) resembles the formula used by Gromov in [27, 8.3.B]. He starts with the set of all bi-infinite geodesics in a hyperbolic metric space $X$, i.e. each geodesic comes equipped with an embedding into $X$. Given two points $x$ and $y$ lying on two bi-infinite geodesics, one can view them as lying in $X$, measure the distance between them and apply (16) to define a metric on the disjoint union of all geodesics. In general there are many geodesics connecting points $a, b \in \partial X$, and the metric is used to identify all of them into one by a quasiisometric homeomorphism (not necessarily an isometry). In that construction, $\mathbb{R}$ does not necessarily act on lines by isometries.

In the construction of this paper we start with an arbitrary metric space $X$. Geodesics are of finite length and are abstractly assigned to each pair of points; no embedding into the space is given. (There might be no embedding at all, the space may be even discrete!) This is why it was important to construct $d^{\times}$first, and this was done using $\beta^{\times}$and the double difference in $X$. The advantage of this formal approach is that for each pair $a, b \in X$ there is a canonically associated line in $\otimes X$ that depends continuously on $a$ and $b$. Moreover, we will see that when $X$ is a hyperbolic complex, this construction extends continuously to the ideal boundary of $X$ so that $\mathbb{R}$ acts by isometries on each ideal line. We will also define and use the $\mathbb{R}$-action on both finite and infinite lines.

Theorem 15 For each $r \in \mathbb{R}$, the map $r^{+}:\left(\otimes X, d_{*}\right) \rightarrow\left(\otimes X, d_{*}\right)$ is a biLipschitz homeomorphism with constant $e^{|r|}$.

Proof For all $v, r \in \mathbb{R}, e^{-|v-r|} \leq e^{|r|-|v|}=e^{|r|} e^{-|v|}$, hence using the substitution $v=u+r$,

$$
\begin{aligned}
& d_{*}\left(r^{+} x, r^{+} y\right)=\int_{-\infty}^{\infty} d^{\times}\left(u^{+} r^{+} x, u^{+} r^{+} y\right) \frac{e^{-|u|}}{2} d u \\
& =\int_{-\infty}^{\infty} d^{\times}\left((u+r)^{+} x,(u+r)^{+} y\right) \frac{e^{-|u|}}{2} d u=\int_{-\infty}^{\infty} d^{\times}\left(v^{+} x, v^{+} y\right) \frac{e^{-|v-r|}}{2} d v \\
& \leq e^{|r|} \int_{-\infty}^{\infty} d^{\times}\left(v^{+} x, v^{+} y\right) \frac{e^{-|v|}}{2} d v=e^{|r|} d_{*}(x, y),
\end{aligned}
$$

and similarly for the inverse map $(-r)^{+}$.

\subsection{Relation between $d^{\times}$and $d_{*}$}

Define a function $\varphi=\varphi_{X}: \circledast X \rightarrow \otimes X$ by

$$
\varphi(x):=\int_{-\infty}^{\infty} r^{+} x \frac{e^{-|r|}}{2} d r .
$$


Here $x \in \llbracket a, a^{\prime} \rrbracket$ and we view $\llbracket a, a^{\prime} \rrbracket$ as a subinterval $\left[\alpha, \alpha^{\prime}\right] \subseteq \mathbb{R}$ as in 2.2 to make sense of the integral.

Theorem 16 Let $X$ be any metric space and define $d^{\times}, d_{*}$ and $\varphi$ as above.

(a) $\varphi$ is a well-defined canonical surjection $\left(\circledast X, d_{*}\right) \rightarrow\left(\circledast X, d^{\times}\right)$whose restriction to each line $\left(\llbracket a, a^{\prime} \rrbracket, d_{*}\right)$ is an isometry onto $\left(\llbracket a, a^{\prime} \rrbracket, d^{\times}\right)$. In particular, each line $\llbracket a, a^{\prime} \rrbracket$ in $\otimes X$ can be parameterized to become a $d_{*}$-geodesic from a to $a^{\prime}$.

(b) The restriction of $\varphi$ to $X$ is the identity map $\left(X, d_{*}\right) \rightarrow\left(X, d^{\times}\right)$, and it is an isometry.

(c) $d_{*}, d^{\times}$and $d$ coincide on $X$, i.e. the canonical embeddings $(X, d) \hookrightarrow$ $\left(\otimes X, d^{\times}\right)$and $(X, d) \hookrightarrow\left(\otimes X, d_{*}\right)$ are isometric.

Proof (a) Each $\varphi(x)$ is well-defined as a real number because $\llbracket a, a^{\prime} ; \cdot \rrbracket^{\prime}$ is non-expanding.

If $x \in \llbracket a, a^{\prime} \rrbracket \cap X$, then $\mathbb{R}$ fixes $x$ and

$$
\varphi(x)=\int_{-\infty}^{\infty} x \frac{e^{-|r|}}{2} d r=x
$$

so $\varphi(x)$ is well-defined and equals $x$, regardless of the choice of $\llbracket a, a^{\prime} \rrbracket$. Therefore $\varphi$ is identity on $X$.

Now we assume $x \in \llbracket a, a^{\prime} \rrbracket \backslash X$, i.e. $x \in \rrbracket a, a^{\prime} \llbracket$ and $a \neq a^{\prime}$. Let the function $\llbracket a, a^{\prime} ; \cdot \rrbracket^{\prime \prime}: \mathbb{R} \rightarrow \llbracket a, a^{\prime} \rrbracket$ be the smooth-out of $\llbracket a, a^{\prime} ; \cdot \rrbracket^{\prime}$, i.e.

$$
\llbracket a, a^{\prime} ; s \rrbracket^{\prime \prime}:=\int_{-\infty}^{\infty} \llbracket a, a^{\prime} ; r+s \rrbracket^{\prime} \frac{e^{-|r|}}{2} d r .
$$

Since $a \neq a^{\prime}$, then $\llbracket a, a^{\prime} ; \cdot \rrbracket^{\prime}: \overline{\mathbb{R}} \rightarrow \llbracket a, a^{\prime} \rrbracket$ is a homeomorphism, and by definition $\left.\varphi\right|_{\llbracket a, a^{\prime} \rrbracket}$ equals the composition $\llbracket a, a^{\prime} ; \cdot \rrbracket^{\prime \prime} \circ\left(\llbracket a, a^{\prime} ; \cdot \rrbracket^{\prime}\right)^{-1}$. By Lemma 2 applied to the function $\llbracket a, a^{\prime} ; \rrbracket^{\prime}, \llbracket a, a^{\prime} ; \cdot \rrbracket^{\prime \prime}$ is a homeomorphism from $\overline{\mathbb{R}}$ onto $\llbracket a, a^{\prime} \rrbracket$, therefore $\varphi$ maps $\llbracket a, a^{\prime} \rrbracket$ homeomorphically onto itself.

Pick any $x, y \in \llbracket a, a^{\prime} \rrbracket$ with $x \leq y$. By Lemma $5 r^{+} x \leq r^{+} y$ for all $r \in \mathbb{R}$, hence $\varphi(x) \leq \varphi(y)$. By Theorem 13(b),

$$
\begin{aligned}
& d^{\times}(\varphi(x), \varphi(y))=\varphi(y)-\varphi(x)=\int_{-\infty}^{\infty}\left(r^{+} y-r^{+} x\right) \frac{e^{-|r|}}{2} d r \\
& =\int_{-\infty}^{\infty} d^{\times}\left(r^{+} y, r^{+} x\right) \frac{e^{-|r|}}{2} d r=d_{*}(x, y),
\end{aligned}
$$


hence $\varphi:\left(\llbracket a, a^{\prime} \rrbracket, d_{*}\right) \rightarrow\left(\llbracket a, a^{\prime} \rrbracket, d^{\times}\right)$is an isometry.

(b) In particular, $d^{\times}\left(a, a^{\prime}\right)=d^{\times}\left(\varphi(a), \varphi\left(a^{\prime}\right)\right)=d_{*}\left(a, a^{\prime}\right)$ for all $a, a^{\prime} \in X$, so the restriction $\varphi:\left(X, d_{*}\right) \rightarrow\left(X, d^{\times}\right)$is an isometry.

(c) This follows from (b) and Theorem 13(c).

Lemma 17 For all $a, a^{\prime} \in X$,

(a) the identity map $\left(\llbracket a, a^{\prime} \rrbracket, d^{\times}\right) \rightarrow\left(\llbracket a, a^{\prime} \rrbracket, d_{*}\right)$ is a homeomorphism and

(b) if $a \neq a^{\prime}$, then $\llbracket a, a^{\prime} ; \rrbracket^{\prime}: \overline{\mathbb{R}} \rightarrow\left(\llbracket a, a^{\prime} \rrbracket, d_{*}\right)$ is a homeomorphism.

Proof (a) If $a=a^{\prime}$, then the statement is obvious. Now assume $a \neq a^{\prime}$. Consider the composition

$$
\overline{\mathbb{R}} \stackrel{\llbracket a, a^{\prime} ; \rrbracket^{\prime}}{\longrightarrow}\left(\llbracket a, a^{\prime} \rrbracket, d^{\times}\right) \stackrel{i d}{\longrightarrow}\left(\llbracket a, a^{\prime} \rrbracket, d_{*}\right) \stackrel{\varphi}{\rightarrow}\left(\llbracket a, a^{\prime} \rrbracket, d^{\times}\right),
$$

where $\varphi$ is the isometry from Theorem 16(a). The first map is a homeomorphism, and one checks that the composition is given by the formula

$$
s \mapsto \int \llbracket a, a^{\prime} ; r+s \rrbracket^{e^{-|r|}} \frac{{ }^{-}}{2} d r,
$$

hence it is the map $\llbracket a, a^{\prime} ; \cdot \rrbracket^{\prime \prime}$ in (19), which is, again, a homeomorphism by Lemma 2 applied to the function $\llbracket a, a^{\prime} ; \cdot \rrbracket^{\prime}$. This implies that $\left(\llbracket a, a^{\prime} \rrbracket, d^{\times}\right) \stackrel{\text { id }}{\longrightarrow}$ $\left(\llbracket a, a^{\prime} \rrbracket, d_{*}\right)$ is a homeomorphism.

(b) The map $\llbracket a, a^{\prime} ; \cdot \rrbracket^{\prime}: \overline{\mathbb{R}} \rightarrow\left(\llbracket a, a^{\prime} \rrbracket, d^{\times}\right)$is the composition of homeomorphisms

$$
\overline{\mathbb{R}} \stackrel{\llbracket a, a^{\prime} ; \rrbracket^{\prime}}{\longrightarrow}\left(\llbracket a, a^{\prime} \rrbracket, d^{\times}\right) \stackrel{i d}{\rightarrow}\left(\llbracket a, a^{\prime} \rrbracket, d_{*}\right) .
$$

Lemma 18 For all $x, y \in \otimes X,\left|d_{*}(x, y)-d^{\times}(x, y)\right| \leq 2$. In particular, $d_{*}$ and $d^{\times}$are ${ }^{+}$equivalent.

Proof By (17),

$$
\begin{aligned}
d_{*}(x, y) & =\int_{-\infty}^{\infty} d^{\times}\left(r^{+} x, r^{+} y\right) \frac{e^{-|r|}}{2} d r \\
\leq & \int_{-\infty}^{\infty}\left(d^{\times}(x, y)+2|r|\right) \frac{e^{-|r|}}{2} d r=d^{\times}(x, y)+2 .
\end{aligned}
$$

Similarly,

$$
\begin{aligned}
d_{*}(x, y) & =\int_{-\infty}^{\infty} d^{\times}\left(r^{+} x, r^{+} y\right) \frac{e^{-|r|}}{2} d r \\
& \geq \int_{-\infty}^{\infty}\left(d^{\times}(x, y)-2|r|\right) \frac{e^{-|r|}}{2} d r=d^{\times}(x, y)-2 .
\end{aligned}
$$




\subsection{The functor $\circledast$ and embeddings into geodesic spaces}

$\otimes$ is a functor on the category of topological spaces: to every topological space $X$ it associates the topological space $\otimes X . \otimes$ is also a functor on the category of metric spaces: to every metric space $(X, d)$ it associates the space $\otimes X$ with the metric $\oplus d$ as in 3.3 . We are intentionally vague about the choice of morphisms here - it is an interesting educational question how $\otimes$ behaves under continuous, Lipschitz and other maps, but this will not be addressed in this article. At the very least, since the construction is canonical, $\otimes$ is functorial with respect to isometries.

As an illustration for the use of functor $\otimes$ we prove the following fact.

Proposition 19 Every metric space $\left(X, d_{X}\right)$ isometrically embeds into a geodesic metric space $\left(Y, d_{Y}\right)$. Moreover, $Y$ can be chosen so that each $g \in \operatorname{Isom}(X)$ extends to an isometry $g^{\prime}$ of $Y$, and the map $\operatorname{Isom}(X) \rightarrow \operatorname{Isom}(Y), g \mapsto g^{\prime}$, is a group monomorphism.

Proof Define $\otimes^{0} X:=X$ and inductively $\otimes^{i} X:=\otimes\left(\otimes^{i-1} X\right)$. Then $\otimes^{i} X$ is a metric space with the metric $d_{i}:=\otimes^{i} d_{X}$ defined inductively from the metric on $X$. By Theorem[16](c) there are canonical isometric embeddings $\circledast^{i-1} X \hookrightarrow \otimes^{i} X$, therefore the union

$$
Y:=\otimes^{\infty}(X):=\bigcup_{i=0}^{\infty} \otimes^{i} X
$$

can be given a metric $d_{Y}$ which restricts to $d_{i}$ on each $\circledast^{i} X$. By Theorem [16(b), each pair of points $a, b \in \otimes^{i-1} X$ is connected by a geodesic in $\otimes^{i} X$, so $Y$ is geodesic. By Theorem [14, each isometry $g$ of $X$ induces an isometry of $\otimes X$. This gives a homomorphism $\operatorname{Isom}(X) \rightarrow \operatorname{Isom}(\otimes X)$ which is clearly injective. Inductively, $g$ extends to an isometry of $\oplus^{i} X$, giving a monomorphism $\operatorname{Isom}(X) \hookrightarrow \operatorname{Isom}\left(\oplus^{i} X\right)$ for each $i$, and therefore to an isometry of $Y$ giving a monomorphism $\operatorname{Isom}(X) \hookrightarrow \operatorname{Isom}(Y)$.

\section{The metric $d_{*}$ and the topology of $* X$}

The goal of this section is to prove the following.

Proposition 20 Let $(X, d)$ be any metric space. The metric $d_{*}$ from 3.3 induces the original topology on the open symmetric join $x_{0}^{*} X$ described in 2.4. Equivalently, the map from 2.4 viewed as

$$
\rrbracket \cdot, \cdot ; \cdot \llbracket^{\prime}:\left(X^{2} \backslash \Delta\right) \times \mathbb{R} \rightarrow\left(x_{0}^{*} X, d_{*}\right)
$$


is a homeomorphism, where $\Delta$ is the diagonal of $X^{2}$.

The proof requires some technical arguments, the reader might want to skip this section at first reading.

Lemma 21 For all $x, y \in[0, \infty), \quad\left|e^{-y}-e^{-x}\right| \leq|y-x|$.

Proof One easily checks that $1-e^{z} \leq-z$ for all $z$. We can assume $x \leq y$. Then $\left|e^{-y}-e^{-x}\right|=e^{-x}\left|1-e^{x-y}\right| \leq\left|1-e^{x-y}\right|=1-e^{x-y} \leq y-x=|y-x|$.

Lemma 22 Let $(X, d)$ be any metric space. Then for all $a, a^{\prime}, b, b^{\prime} \in X$ and $s, t \in \overline{\mathbb{R}}$,

(a) $\quad d^{\times}\left(\llbracket a, a^{\prime} ; s \rrbracket, \llbracket b, b^{\prime} ; t \rrbracket\right) \leq|t-s|+2 d(a, b)+2 d\left(a^{\prime}, b^{\prime}\right)$,

(b) $\quad d^{\times}\left(\llbracket a, a^{\prime} ; s \rrbracket^{\prime}, \llbracket b, b^{\prime} ; t \rrbracket^{\prime}\right) \leq|t-s|+4 d(a, b)+4 d\left(a^{\prime}, b^{\prime}\right)$,

(c) $\quad d_{*}\left(\llbracket a, a^{\prime} ; s \rrbracket^{\prime}, \llbracket b, b^{\prime} ; t \rrbracket^{\prime}\right) \leq|t-s|+4 d(a, b)+4 d\left(a^{\prime}, b^{\prime}\right)$.

Proof (a) For any $u \in X$, by the definition of $\beta^{\times}$and triangle inequality,

$$
\begin{aligned}
& \left|\beta_{u}^{\times}\left(\llbracket a, a^{\prime} ; s \rrbracket, \llbracket b, b^{\prime} ; t \rrbracket\right)\right|=\left|\left\langle a \mid a^{\prime}\right\rangle_{u}+\right| s-\left\langle a, a^{\prime} \mid u\right\rangle\left|-\left\langle b \mid b^{\prime}\right\rangle_{u}-\right| t-\left\langle b, b^{\prime} \mid u\right\rangle|| \\
& \leq|| s-\left\langle a, a^{\prime} \mid u\right\rangle|-| t-\left\langle b, b^{\prime} \mid u\right\rangle||+\left|\left\langle a \mid a^{\prime}\right\rangle_{u}-\left\langle b \mid b^{\prime}\right\rangle_{u}\right| \\
& \leq\left|s-\left\langle a, a^{\prime} \mid u\right\rangle-t+\left\langle b, b^{\prime} \mid u\right\rangle\right|+\left|\left\langle a \mid a^{\prime}\right\rangle_{u}-\left\langle b \mid b^{\prime}\right\rangle_{u}\right| \\
& \leq|t-s|+\left|\left\langle a, a^{\prime} \mid u\right\rangle-\left\langle b, b^{\prime} \mid u\right\rangle\right|+\left|\left\langle a \mid a^{\prime}\right\rangle_{u}-\left\langle b \mid b^{\prime}\right\rangle_{u}\right| \\
& \leq|t-s|+|\langle a, b \mid u\rangle|+\left|\left\langle a^{\prime}, b^{\prime} \mid u\right\rangle\right|+\left|\left\langle a \mid a^{\prime}\right\rangle_{u}-\left\langle b \mid a^{\prime}\right\rangle_{u}\right|+\left|\left\langle b \mid a^{\prime}\right\rangle_{u}-\left\langle b \mid b^{\prime}\right\rangle_{u}\right| \\
& \leq|t-s|+2 d(a, b)+2 d\left(a^{\prime}, b^{\prime}\right) .
\end{aligned}
$$

Then by the definition of $d^{\times}$,

$d^{\times}\left(\llbracket a, a^{\prime} ; s \rrbracket, \llbracket b, b^{\prime} ; t \rrbracket\right)=\sup _{u \in X}\left|\beta_{u}^{\times}\left(\llbracket a, a^{\prime} ; s \rrbracket, \llbracket b, b^{\prime} ; t \rrbracket\right)\right| \leq|t-s|+2 d(a, b)+2 d\left(a^{\prime}, b^{\prime}\right)$.

(b) Recall from 2.2 that $\llbracket a, a^{\prime} \rrbracket$ is a copy of the interval $\left[\alpha, \alpha^{\prime}\right] \subseteq \mathbb{R}$, where $\alpha:=-\left\langle a^{\prime} \mid x_{0}\right\rangle_{a}$ and $\alpha^{\prime}:=\left\langle a \mid x_{0}\right\rangle_{a^{\prime}}$. Similarly, $\llbracket b, b^{\prime} \rrbracket$ is a copy of $\left[\beta, \beta^{\prime}\right] \subseteq \mathbb{R}$ where $\beta:=-\left\langle b^{\prime} \mid x_{0}\right\rangle_{b}$ and $\beta^{\prime}:=\left\langle b \mid x_{0}\right\rangle_{b^{\prime}}$. By the definition of $\langle\cdot \mid \cdot\rangle$. and triangle inequality,

$$
\begin{aligned}
& |\beta-\alpha|=\left|\left\langle a^{\prime} \mid x_{0}\right\rangle_{a}-\left\langle b^{\prime} \mid x_{0}\right\rangle_{b}\right| \leq\left|d\left(a, a^{\prime}\right)-d\left(b, b^{\prime}\right)\right| / 2 \\
& +\left|d\left(a, x_{0}\right)-d\left(b, x_{0}\right)\right| / 2+\left|d\left(b^{\prime}, x_{0}\right)-d\left(a^{\prime}, x_{0}\right)\right| / 2 \leq d(a, b)+d\left(a^{\prime}, b^{\prime}\right),
\end{aligned}
$$

and similarly $\left|\beta^{\prime}-\alpha^{\prime}\right| \leq d(a, b)+d\left(a^{\prime}, b^{\prime}\right)$. It follows from the definition of $\theta$ that

$$
\left|\theta\left[\beta, \beta^{\prime} ; t\right]-\theta\left[\alpha, \alpha^{\prime} ; t\right]\right| \leq \max \left\{|\beta-\alpha|,\left|\beta^{\prime}-\alpha^{\prime}\right|\right\} \leq d(a, b)+d\left(a^{\prime}, b^{\prime}\right) .
$$


Using Lemma 3 we denote

$$
\begin{aligned}
& A:=\theta^{\prime}\left[\alpha, \alpha^{\prime} ; t\right]=\theta\left[\alpha, \alpha^{\prime} ; t\right]+\left(e^{-|t-\alpha|}-e^{-\left|t-\alpha^{\prime}\right|}\right) / 2 \text { and } \\
& B:=\theta^{\prime}\left[\beta, \beta^{\prime} ; t\right]=\theta\left[\beta, \beta^{\prime} ; t\right]+\left(e^{-|t-\beta|}-e^{-\left|t-\beta^{\prime}\right|}\right) / 2,
\end{aligned}
$$

then by (21), Lemma 21] and (201),

$$
\begin{aligned}
& |B-A| \\
& \leq\left|\theta\left[\beta, \beta^{\prime} ; t\right]-\theta\left[\alpha, \alpha^{\prime} ; t\right]\right|+\left|e^{-|t-\beta|}-e^{-|t-\alpha|}\right| / 2+\left|e^{-\left|t-\alpha^{\prime}\right|}-e^{-\left|t-\beta^{\prime}\right|}\right| / 2 \\
& \leq d(a, b)+d\left(a^{\prime}, b^{\prime}\right)+|| t-\beta|-| t-\alpha|| / 2+|| t-\alpha^{\prime}|-| t-\beta^{\prime}|| / 2 \\
& \leq d(a, b)+d\left(a^{\prime}, b^{\prime}\right)+|\beta-\alpha| / 2+\left|\beta^{\prime}-\alpha^{\prime}\right| / 2 \leq 2 d(a, b)+2 d\left(a^{\prime}, b^{\prime}\right) .
\end{aligned}
$$

By (7), part (a) and the above inequality,

$$
\begin{aligned}
& d^{\times}\left(\llbracket a, a^{\prime} ; t \rrbracket^{\prime}, \llbracket b, b^{\prime} ; t \rrbracket^{\prime}\right)=d^{\times}\left(\llbracket a, a^{\prime} ; \theta^{\prime}\left[\alpha, \alpha^{\prime} ; t\right] \rrbracket, \llbracket b, b^{\prime} ; \theta^{\prime}\left[\beta, \beta^{\prime} ; t\right] \rrbracket\right) \\
& =d^{\times}\left(\llbracket a, a^{\prime} ; A \rrbracket, \llbracket b, b^{\prime} ; B \rrbracket\right) \leq|B-A|+2 d(a, b)+2 d\left(a^{\prime}, b^{\prime}\right) \\
& \leq 4 d(a, b)+4 d\left(a^{\prime}, b^{\prime}\right) .
\end{aligned}
$$

Since the map $\llbracket a, a^{\prime} ; \cdot \rrbracket^{\prime}: \mathbb{R} \rightarrow\left(\llbracket a, a^{\prime} \rrbracket, d^{\times}\right)$is non-expanding,

$$
\begin{aligned}
& d^{\times}\left(\llbracket a, a^{\prime} ; s \rrbracket^{\prime}, \llbracket b, b^{\prime} ; t \rrbracket^{\prime}\right) \leq d^{\times}\left(\llbracket a, a^{\prime} ; s \rrbracket^{\prime}, \llbracket a, a^{\prime} ; t \rrbracket^{\prime}\right)+d^{\times}\left(\llbracket a, a^{\prime} ; t \rrbracket^{\prime}, \llbracket b, b^{\prime} ; t \rrbracket^{\prime}\right) \\
& \leq|t-s|+4 d(a, b)+4 d\left(a^{\prime}, b^{\prime}\right) .
\end{aligned}
$$

(c) This follows from (b) and the definition of $d_{*}$ :

$$
\begin{aligned}
& d_{*}\left(\llbracket a, a^{\prime} ; s \rrbracket^{\prime}, \llbracket b, b^{\prime} ; t \rrbracket^{\prime}\right)=\int_{-\infty}^{\infty} d^{\times}\left(\llbracket a, a^{\prime} ; r+s \rrbracket^{\prime}, \llbracket b, b^{\prime} ; r+t \rrbracket^{\prime}\right) \frac{e^{-|r|}}{2} d r \\
& \leq \int_{-\infty}^{\infty}\left(|(r+t)-(r+s)|+4 d(a, b)+4 d\left(a^{\prime}, b^{\prime}\right)\right) \frac{e^{-|r|}}{2} d r \\
& =|t-s|+4 d(a, b)+4 d\left(a^{\prime}, b^{\prime}\right) .
\end{aligned}
$$

Lemma 23 The function $\omega:[0, \infty) \rightarrow[0, \infty)$ defined by $\omega(\tau):=\tau+2 e^{-\tau / 2}-2$ is a homeomorphism. The obvious extension $\omega:[0, \infty] \rightarrow[0, \infty]$ is also a homeomorphism.

Proof This follows from the facts that $\omega(0)=0, \frac{\partial \omega}{\partial \tau}(\tau)>0$ for $\tau>0$, and $\omega(\tau) \rightarrow \infty$ as $\tau \rightarrow \infty$.

Lemma 24 For all $x, y \in \circledast X, d^{\times}(x, y) \leq \omega^{-1}\left(d_{*}(x, y)\right)$, where $\omega$ is from Lemma 23.

Moreover, for all $x, y \in \otimes X$ and $r \in \mathbb{R}, d^{\times}\left(r^{+} x, r^{+} y\right) \leq \omega^{-1}\left(e^{|r|} d_{*}(x, y)\right)$. 
Proof Denote $\tau:=d^{\times}(x, y)$. Since the map $\llbracket a, b ; \cdot \rrbracket^{\prime}$ is non-expanding, we have $d^{\times}\left(r^{+} x, r^{+} y\right) \geq d^{\times}(x, y)-d^{\times}\left(x, r^{+} x\right)-d^{\times}\left(y, r^{+} y\right) \geq d^{\times}(x, y)-2|r|=\tau-2|r|$

for all $r \in \mathbb{R}$, then

$$
\begin{aligned}
& d_{*}(x, y)=\int_{-\infty}^{\infty} d^{\times}\left(r^{+} x, r^{+} y\right) \frac{e^{-|r|}}{2} d r \geq \int_{-\tau / 2}^{\tau / 2}(\tau-2|r|) \frac{e^{-|r|}}{2} d r \\
& =\tau+2 e^{-\tau / 2}-2=\omega(\tau)=\omega\left(d^{\times}(x, y)\right) .
\end{aligned}
$$

Since $\omega$ is increasing, $d^{\times}(x, y) \leq \omega^{-1}\left(d_{*}(x, y)\right)$ for any $x$ and $y$ in $\oplus X$. Applying the inequality (22) to $r^{+} x$ and $r^{+} y$ and using Theorem [15,

$$
\omega\left(d^{\times}\left(r^{+} x, r^{+} y\right)\right) \leq d_{*}\left(r^{+} x, r^{+} y\right) \leq e^{|r|} d_{*}(x, y),
$$

therefore $d^{\times}\left(r^{+} x, r^{+} y\right) \leq \omega^{-1}\left(e^{|r|} d_{*}(x, y)\right)$.

Lemma 25 Let $a \in X$ and $x_{i}=\llbracket a_{i}, a_{i}^{\prime} ; s_{i} \rrbracket^{\prime}$ be a sequence in $\otimes X$ such that $d_{*}\left(x_{i}, a\right) \rightarrow 0$ as $i \rightarrow \infty$. Then $d\left(a_{i}, a\right) \rightarrow 0$ or $d\left(a_{i}^{\prime}, a\right) \rightarrow 0$ as $i \rightarrow \infty$.

Proof Suppose not, then after taking a subsequence there exists $\varepsilon>0$ such that $d^{\times}\left(a_{i}, a\right)=d\left(a_{i}, a\right)>\varepsilon$ and $d^{\times}\left(a_{i}^{\prime}, a\right)=d\left(a_{i}^{\prime}, a\right)>\varepsilon$ for all $i$. Since $d_{*}\left(x_{i}, a\right) \rightarrow 0$, then by Lemma 24 $d^{\times}\left(x_{i}, a\right) \rightarrow 0$, so extracting a subsequence again we can assume that

$$
d^{\times}\left(x_{i}, a\right) \leq \varepsilon / 4 \quad \text { for all } i .
$$

For each $i$ there is a point $y_{i}=\llbracket a_{i}, a_{i}^{\prime} ; t_{i} \rrbracket^{\prime}$ such that

$$
t_{i}>s_{i} \quad \text { and } \quad d^{\times}\left(x_{i}, y_{i}\right)=\varepsilon / 2 .
$$

In particular,

$$
d^{\times}\left(a, y_{i}\right) \geq d^{\times}\left(x_{i}, y_{i}\right)-d^{\times}\left(x_{i}, a\right) \geq \varepsilon / 2-\varepsilon / 4=\varepsilon / 4 .
$$

Let $z \in \llbracket a_{i}, a_{i}^{\prime} \rrbracket$ represent an arbitrary point that lies between $x_{i}$ and $y_{i}$, then

$$
\begin{aligned}
& d^{\times}(a, z) \leq d^{\times}\left(a, x_{i}\right)+d^{\times}\left(x_{i}, z\right) \leq \varepsilon / 4+\varepsilon / 2=3 \varepsilon / 4, \quad \text { hence } \\
& d^{\times}\left(z, a_{i}\right)>\varepsilon / 4 \quad \text { and } \quad d^{\times}\left(z, a_{i}^{\prime}\right)>\varepsilon / 4 .
\end{aligned}
$$

Take $\epsilon>0$ sufficiently small so that

$$
\epsilon<\varepsilon / 4 \quad \text { and } \quad \epsilon<\left(1-e^{-\varepsilon}\right) / 2 .
$$

By the above,

$$
d^{\times}\left(z, a_{i}\right)>\varepsilon / 4>\epsilon \quad \text { and } \quad d^{\times}\left(z, a_{i}^{\prime}\right)>\varepsilon / 4>\epsilon \quad \text { for all } i .
$$


Let $\alpha_{i} \leq \alpha_{i}^{\prime}$ be the end-point coordinates of $\llbracket a_{i}, a_{i}^{\prime} \rrbracket$. Since

$$
\begin{aligned}
& \alpha_{i}^{\prime}-\alpha_{i}=d^{\times}\left(a_{i}, a_{i}^{\prime}\right)=d^{\times}\left(a_{i}, x_{i}\right)+d^{\times}\left(x_{i}, a_{i}^{\prime}\right) \\
& \geq\left(d^{\times}\left(a_{i}, a\right)-\varepsilon / 4\right)+\left(d^{\times}\left(a_{i}^{\prime}, a\right)-\varepsilon / 4\right) \geq 2(\varepsilon-\varepsilon / 4)>\varepsilon,
\end{aligned}
$$

we have

$$
0<\epsilon<\left(1-e^{-\varepsilon}\right) / 2 \leq\left(1-e^{\alpha_{i}-\alpha_{i}^{\prime}}\right) / 2 \quad \text { for all } i .
$$

Equations (24) and (25) show that Lemma 4 applies to the map $\llbracket a_{i}, a_{i}^{\prime} ; \cdot \rrbracket^{\prime}: \mathbb{R} \rightarrow$ $\left(\llbracket a_{i}, a_{i}^{\prime} \rrbracket, d^{\times}\right)$, therefore the derivative of this map in the interval $\left[s_{i}, t_{i}\right]$ is at least $\epsilon$. Then

$$
t_{i}-s_{i} \leq \frac{d^{\times}\left(x_{i}, y_{i}\right)}{\epsilon}=\frac{\varepsilon}{2 \epsilon} .
$$

Denote $R:=\varepsilon /(2 \epsilon)$ and $r_{i}:=t_{i}-s_{i} \in[0, R]$, so we have $y_{i}=r_{i}{ }^{+} x_{i}$. By Lemma 24.

$$
\begin{aligned}
& d^{\times}\left(a, y_{i}\right)=d^{\times}\left(a, r_{i}{ }^{+} x_{i}\right)=d^{\times}\left(r_{i}{ }^{+} a, r_{i}{ }^{+} x_{i}\right) \\
& \quad \leq \omega^{-1}\left(e^{r_{i}} d_{*}\left(a, x_{i}\right)\right) \leq \omega^{-1}\left(e^{R} d_{*}\left(a, x_{i}\right)\right) \underset{i \rightarrow \infty}{\rightarrow} 0 .
\end{aligned}
$$

This contradicts (23).

Proof of Proposition [20] Recall from 2.4 that the topology on $x_{0}^{*} X \subseteq{ }_{x_{0}} X$ is induced by the bijection $\rrbracket \cdot, \cdot ; \cdot \mathbb{I}^{\prime}:\left(X^{2} \backslash \Delta\right) \times \mathbb{R} \rightarrow x_{0}^{*} X$. Since the set $\left(X^{2} \backslash \Delta\right) \times \mathbb{R}$ is open in $X^{2} \times \overline{\mathbb{R}}$, the topology of $\left(X^{2} \backslash \Delta\right) \times \mathbb{R}$ is locally the product of the topologies on $X, X$, and $\mathbb{R}$. Therefore a typical neighborhood of $x=\rrbracket a, a^{\prime} ; s \rrbracket^{\prime}$ in $x_{0}^{*} X$ is of the form $N(x, \varepsilon):=\rrbracket B_{\varepsilon}, B_{\varepsilon}^{\prime} ; I_{\varepsilon} \llbracket^{\prime}$, where $B_{\varepsilon}$ and $B_{\varepsilon}^{\prime}$ are disjoint open balls in $(X, d)$ of radius $\varepsilon$ centered at $a$ and $a^{\prime}$, respectively, and $I_{\varepsilon}=$ $(s-\varepsilon, s+\varepsilon) \subseteq \mathbb{R}$.

Our goal is to show that the identity map $x_{0}^{*} X \rightarrow\left(x_{0}^{*} X, d_{*}\right)$ is a homeomorphism. Let $y=\rrbracket b, b^{\prime} ; t \llbracket^{\prime}$ represent an arbitrary point in $N(x, \varepsilon)$, then $|t-s|<\varepsilon$, $d(a, b)<\varepsilon, d\left(a^{\prime}, b^{\prime}\right)<\varepsilon$.

Given any $\epsilon>0$, we let $\varepsilon:=\epsilon / 9$, then Lemma 22(c) implies

$d_{*}(x, y)=d_{*}\left(\rrbracket a, a^{\prime} ; s \rrbracket^{\prime}, \rrbracket b, b^{\prime} ; t \rrbracket^{\prime}\right) \leq|t-s|+4 d(a, b)+4 d\left(a^{\prime}, b^{\prime}\right)<\varepsilon+4 \varepsilon+4 \varepsilon=\epsilon$ i.e. $N(x, \varepsilon) \subseteq B_{d_{*}}(x, \epsilon)$. This shows that the identity map ${ }_{x_{0}^{*}}^{*} X \rightarrow\left({ }_{x_{0}^{*}}^{*} X, d_{*}\right)$ is continuous at $x$.

Suppose that the inverse identity map $\left({ }_{x_{0}}^{*} X, d_{*}\right) \rightarrow{ }_{x_{0}^{*}}^{*} X$ is not continuous at $x=\rrbracket a, a^{\prime} ; s \rrbracket^{\prime}$, then there exist a product neigbourhood $N(x, \varepsilon)=\rrbracket B_{\varepsilon}, B_{\varepsilon}^{\prime} ; I_{\varepsilon} \rrbracket^{\prime}$ of $x$ and a sequence $\left\{x_{i}\right\}$ such that

$$
d_{*}\left(x_{i}, x\right) \underset{i \rightarrow \infty}{\rightarrow} 0 \quad \text { and } \quad x_{i} \in(* X) \backslash N(x, \varepsilon) .
$$


We have $x_{i}=\rrbracket a_{i}, a_{i}^{\prime} ; s_{i} \llbracket^{\prime}$ for some $a_{i}, a_{i}^{\prime} \in X$ and $s_{i} \in \mathbb{R}$.

Since $d_{*}\left(x_{i}, x\right) \rightarrow 0$, using Lemma 24, for any $j>0$ there exists $i=i(j)$ such that

$$
d_{*}\left(j^{+} x_{i}, j^{+} x\right) \leq \omega^{-1}\left(e^{|j|} d_{*}\left(x_{i}, x\right)\right) \leq 1 / j .
$$

Therefore the subsequence $\left\{x_{j}:=x_{i(j)}\right\}$ satisfies $d_{*}\left(j^{+} x_{j}, j^{+} x\right) \rightarrow 0$ as $j \rightarrow \infty$. By Lemma 17(b), $d_{*}\left(j^{+} x, a^{\prime}\right) \rightarrow 0$ as $j \rightarrow \infty$, hence

$$
d_{*}\left(j^{+} x_{j}, a^{\prime}\right) \leq d_{*}\left(j^{+} x_{j}, j^{+} x\right)+d_{*}\left(j^{+} x, a^{\prime}\right) \underset{j \rightarrow \infty}{\rightarrow} 0 .
$$

Then by Lemma 25 applied to the sequence $\left\{j^{+} x_{j}\right\}$ we have

$$
a_{j} \rightarrow a^{\prime} \quad \text { or } \quad a_{j}^{\prime} \rightarrow a^{\prime} \quad \text { in } X .
$$

By a similar argument using $(-j)^{+}$in (27) and passing to a subsequence we also deduce that

$$
a_{j} \rightarrow a \quad \text { or } \quad a_{j}^{\prime} \rightarrow a \quad \text { in } X .
$$

Suppose that $a_{j} \rightarrow a^{\prime}$ and $a_{j}^{\prime} \rightarrow a$. Since $\overline{\mathbb{R}}$ is compact, passing to a subsequence we can assume that $s_{j} \rightarrow t$ for some $t \in \overline{\mathbb{R}}$. Lemma 22(c) implies that

$$
\begin{aligned}
& d_{*}\left(x_{j}, \llbracket a^{\prime}, a ; t \rrbracket^{\prime}\right)=d_{*}\left(\llbracket a_{j}, a_{j}^{\prime} ; s_{j} \rrbracket^{\prime}, \llbracket a^{\prime}, a ; t \rrbracket^{\prime}\right) \\
& \quad \leq\left|t-s_{j}\right|+4 d\left(a_{j}, a^{\prime}\right)+4 d\left(a_{j}^{\prime}, a\right) \underset{j \rightarrow \infty}{\rightarrow} 0 .
\end{aligned}
$$

(30) and (26) say that $x_{j}$ converges in metric $d_{*}$ both to $x \in \rrbracket a, a^{\prime} \llbracket$ and to $\llbracket a^{\prime}, a ; t \rrbracket^{\prime}$. This is impossible since $\rrbracket a, a^{\prime} \llbracket$ and $\llbracket a^{\prime}, a \rrbracket$ are disjoint.

The only possibility left is that $a_{j} \rightarrow a$ and $a_{j}^{\prime} \rightarrow a^{\prime}$. After passing to a subsequence we can assume that $a_{j} \in B_{\varepsilon}$ and $a_{j}^{\prime} \in B_{\varepsilon}^{\prime}$ for all $j$. Since $x_{i} \notin$ $N(x, \varepsilon)$ by (26), we must have $\left|s_{j}-s\right| \geq \varepsilon$ for all $j$. After passing to a subsequence we can assume that

$$
s_{j} \rightarrow \bar{s} \quad \text { for some } \bar{s} \in[-\infty, s-\varepsilon] \cup[s+\varepsilon, \infty] .
$$

Then by Lemma 22(c),

$$
\begin{aligned}
& d_{*}\left(x_{j}, \llbracket a^{\prime}, a ; \bar{s} \rrbracket^{\prime}\right)=d_{*}\left(\llbracket a_{j}, a_{j}^{\prime} ; s_{j} \rrbracket^{\prime}, \llbracket a^{\prime}, a ; \bar{s} \rrbracket^{\prime}\right) \\
& \quad \leq\left|\bar{s}-s_{j}\right|+4 d\left(a_{j}, a\right)+4 d\left(a_{j}^{\prime}, a^{\prime}\right) \underset{j \rightarrow \infty}{\longrightarrow} 0 .
\end{aligned}
$$

Then $x_{j}$ converges in the metric $d_{*}$ both to $x=\rrbracket a, a^{\prime} ; s \rrbracket^{\prime}$ and to $\llbracket a^{\prime}, a ; \bar{s} \rrbracket^{\prime}$, which is impossible since the two points are distinct. 


\section{$5 \quad$ Metric complexes and hyperbolic complexes}

Suppose $X$ is a simplicial complex and $d$ is any metric on its 0-skeleton $X^{(0)}$. Let $P\left(X^{(0)}\right)$ be the power set of $X^{(0)}$. Each simplex in $X$ is uniquely determined by its vertices, so the simplicial structure on $X$ can described combinatorially as a collection $\mathcal{U} \subseteq P\left(X^{(0)}\right)$ consisting of finite subsets of $X^{(0)}$ which is subset-closed: $U \in \mathcal{U}$ and $U^{\prime} \subseteq U$ imply $U^{\prime} \in \mathcal{U}$. Moreover, each $U \in \mathcal{U}$ can be viewed as the convex hull of its vertices, that is each point in the topological simplex $\sigma_{U}$ corresponding to $U$ is described uniquely as a linear combination

$$
\sum_{x \in U} \alpha_{x} x \quad \text { where } \quad \alpha_{x} \in[0,1] \text { and } \quad \sum_{x \in U} \alpha_{x}=1 .
$$

It is easy to check that the formula

$$
\widetilde{d}\left(\sum_{x \in U} \alpha_{x} x, \sum_{y \in U} \beta_{y} y\right):=\sum_{x \in U} \sum_{y \in U} \alpha_{x} \beta_{y} d(x, y)
$$

defines a metric $\widetilde{d}$ on $X$ whose restriction to each simplex $\sigma_{U}$ is homeomorphic (even linearly isomorphic) to the standard simplex of dimension $\# U-1$. Moreover, the inclusion $\left(X^{(0)}, d\right) \hookrightarrow(X, \widetilde{d})$ is an isometric embedding, i.e. $\widetilde{d}$ is an extension of $d$. We will omit $\sim$ from the notation of extended metric.

\subsection{The functor $\Psi$ and the canonical word metric $d_{X}$}

Let $X$ be a simplicial complex and $d$ be an arbitrary generalized metric on $X$, $X^{(1)}$ or $X^{(0)}$. Denote $\Psi(d)$ the result of the following procedure: restrict $d$ to $X^{(0)}$ and extend to all of $X$ by linearity formula (31). $\Psi(d)$ is a generalized metric, and $\Psi(d)$ is a metric if and only if $d$ is. If the simplices in $(X, d)$ have uniformly bounded diameters, then the inclusions $\left(X^{(0)}, d\right) \hookrightarrow(X, d)$ and $\left(X^{(0)}, d\right) \hookrightarrow(X, \Psi(d))$ and the identity map $(X, d) \rightarrow(X, \Psi(d))$ are quasiisometries.

For any simplicial complex $X$ there is a canonical choice of generalized metric $d_{X}$, obtained as follows: take the generalized path metric on $X^{(1)}$ defined by assigning length 1 to each edge, and apply functor $\Psi . d_{X}$ is a metric if and only if $X$ is connected; we will call it the word metric on $X$, since this generalizes the word metrics on groups. In general $d_{X}$ is not intrinsic. 


\subsection{Metric complexes}

We will say that a metric $d$ on $X$ is induced from the 0 -skeleton if $d=\Psi(d)$. A metric complex will be a pair $(X, d)$ where $X$ is a simplicial complex and $d$ is a metric that is induced from the 0-skeleton. Examples:

(0) Any metric space is a 0-dimensional metric complex.

(1) Let $\mathcal{G}$ be the Cayley graph of any finitely generated group with respect to a finite generating set. Subdivide $\mathcal{G}$ if needed to make it a simplicial complex. For any metric $d$ on $\mathcal{G}$ quasiisometric to the word metric, $(\mathcal{G}, \Psi(d))$ is a 1-dimensional metric complex, where $\Psi(d)$ is quasiisometric to $d$.

(2) Let $M$ be any compact triangulated smooth $n$-manifold with $\pi_{1}(M)=\Gamma$. Its universal cover $\widetilde{M}$ has the distance function $d$ coming from the Riemannian structure, then $(\widetilde{M}, \Psi(d))$ is an $n$-dimensional metric complex. The identity map $(\widetilde{M}, d) \rightarrow(\widetilde{M}, \Psi(d))$ is a $\Gamma$-invariant homeomorphism and quasiisometry.

(3) Let $M$ be any compact triangulated $n$-manifold with $\pi_{1}(M)=\Gamma$. Let $d$ be any $\Gamma$-invariant metric on $\widetilde{M}$ quasiisometric to the word metric $d_{X}$ defined in 5.1, for example the word metric itself. Then $(\widetilde{M}, \Psi(d))$ is an $n$-dimensional metric complex homeomorphic to $\widetilde{M}$. The metrics $d_{X}$ and $\Psi(d)$ are quasiisometric.

For a metric complex $(X, d)$, Isom $(X, d)$ will denote the group of simplicial automorphisms of $X$ preserving the given metric $d$. If $d$ is the word metric $d_{X}$ on $X$, then we will use the notation $\operatorname{Isom}(X)$ for $\operatorname{Isom}(X, d)$. Since $d_{X}$ is canonical, $\operatorname{Isom}(X)$ is just the group of all simplicial automorphisms of $X$.

\subsection{Hyperbolic complexes}

First let $\mathcal{G}$ be any connected graph. We equip $\mathcal{G}$ with the word metric $d$ which is by definition the path metric induced by assigning length 1 to each edge. A geodesic in $\mathcal{G}$ is an isometric embedding $\alpha: I \rightarrow(\mathcal{G}, d)$ of a closed interval $I \subseteq \mathbb{R}$. Often we will use the same notation for a geodesic and its image. For all $a, b \in \overline{\mathcal{G}}$ we denote $\operatorname{Geod}(a, b)$ the set of all geodesics in $\overline{\mathcal{G}}$ connecting $a$ to $b$, and fix one arbitrary choice of a geodesic $[a, b] \in \operatorname{Geod}(a, b)$. When $a \in \mathcal{G}$, we assume more precisely that $[a, b]$ is the image of the isometric embedding $[a, b ; \cdot]:[0, d(a, b)] \rightarrow \mathcal{G}$ with $[a, b ; 0]=a$ and $[a, b ; d(a, b)]=b$; that is for $t \in[0, d(a, b)],[a, b ; t]$ is the unique point in $[a, b]$ with $d(a,[a, b ; t])=t$. 
Geodesic triangles have canonically defined inscribed triples: for all $a, b, c \in \mathcal{G}$ and any choice of geodesics $\alpha \in \operatorname{Geod}(b, c), \beta \in \operatorname{Geod}(c, a), \gamma \in \operatorname{Geod}(a, b)$ there are unique $\bar{a} \in \alpha, \bar{b} \in \beta, \bar{c} \in \gamma$ with

$$
d(a, \bar{b})=d(a, \bar{c}), \quad d(b, \bar{a})=d(b, \bar{c}), \quad d(c, \bar{a})=d(c, \bar{b}) .
$$

Equivalently, $\bar{a}$ is the unique point in $\alpha$ satisfying $d(b, \bar{a})-d(\bar{a}, c)=d(b, a)-$ $d(a, c)$, and similarly for $\bar{b}$ and $\bar{c}$. It is convenient to think of $\bar{a}$ as of a projection of $a$ to $\alpha$.

A graph $\mathcal{G}$ is hyperbolic if it is connected and there exists $\delta \in[0, \infty)$ so that all geodesic triangles in $\mathcal{G}$ are $\delta$-fine: for all $a, b, c \in \mathcal{G}$ and the inscribed triple $\bar{a}, \bar{b}, \bar{c}$ as above we have

if $x \in \beta, y \in \gamma$ and $d(a, x)=d(a, y) \leq d(a, \bar{b})=d(a, \bar{c})$, then $d(x, y) \leq \delta$.

Each simplicial complex $X$ has the canonical word metric $d=d_{X}$ as in 5.1. A hyperbolic complex will be a uniformly locally finite metric complex whose 1 -skeleton $(\mathcal{G}, d)$ is a hyperbolic graph. Note that it is a part of the definition that $X$ is connected and uniformly locally finite.

Each hyperbolic graph has ideal boundary (see [27, 26, 4, for definitions). Since each hyperbolic complex $X$ is quasiisometric to its 1-skeleton $\mathcal{G}$, this defines the boundary $\partial X:=\partial \mathcal{G}$ and the compactification $\bar{X}:=X \sqcup \partial X$.

We put the generalized metric $|\cdot|$ on $\overline{\mathbb{R}}:=[-\infty, \infty]$ by declaring

$| \pm \infty-r|:=\infty$ for all $r \in \mathbb{R}, \quad|( \pm \infty)-(\mp \infty)|:=\infty, \quad|( \pm \infty)-( \pm \infty)|:=0$,

and put the topology on $\overline{\mathbb{R}}$ that makes it a closed interval. (This topology is not induced by the generalized metric.) Now a geodesic in $\overline{\mathcal{G}}$ is a continuous isometric embedding $\alpha: I \rightarrow \overline{\mathcal{G}}$ of an interval $I \subseteq \overline{\mathbb{R}}$, where isometry is understood as preserving the distance that can take infinite values. For example, the map $\{0\} \rightarrow\{a\}$ for any $a \in \partial \mathcal{G}$ is trivially a geodesic in $\overline{\mathcal{G}}$. Another example is a geodesic ray in $\overline{\mathcal{G}}$ converging to $a \in \partial \mathcal{G}$, i.e. a continuous isometric embedding $\alpha:[0, \infty] \rightarrow \overline{\mathcal{G}}$ with $\alpha(0) \in \overline{\mathcal{G}}$ and $\alpha(\infty)=a$.

\section{The metric $\hat{d}$ and the double difference in $\bar{X}$}

For the rest of the paper $X$ will denote a hyperbolic metric complex and we fix a positive integer $\delta$ such that the geodesic triangles in $\mathcal{G}$ are $\delta$-fine.

The goal of this section is to construct a double difference - a continuous function on quadruples of points in $\bar{X}$ (Theorem [35). It plays the role of the 
logarithm of the absolute value of the classical cross ratio in $\mathbb{C} \cup\{\infty\}=\partial \mathbb{H}^{3}$, but is going to be defined in a much more general setting: both on an arbitrary hyperbolic complex and on its ideal boundary. The double difference will be defined precisely, rather than "up to a bounded amount", as often happened for various notions in hyperbolic groups. First we provide several important auxiliary results.

\subsection{The extended metric $\hat{d}$}

In 31, a metric $\hat{d}$ was constructed on any hyperbolic group $\Gamma$. It was shown that $\hat{d}$ is strongly bolic, $\Gamma$-invariant, quasiisometric to the word metric $d$, and satisfies

Theorem 26 31] There exist constants $C \in[0, \infty)$ and $\mu \in[0,1)$ with the following property. If $a, a^{\prime}, b, b^{\prime} \in \Gamma, d\left(a, a^{\prime}\right) \leq 1$, and $d\left(b, b^{\prime}\right) \leq 1$, then

$$
\left|\hat{d}(a, b)-\hat{d}\left(a^{\prime}, b\right)-\hat{d}\left(a, b^{\prime}\right)+\hat{d}\left(a^{\prime}, b^{\prime}\right)\right| \leq C \mu^{d(a, b)} .
$$

The above theorem is not enough for the purposes of this paper, and just having a strongly bolic invariant metric is not enough either. We will show that actually $\hat{d}$ satisfies stronger properties (Theorem 32).

Now let $X$ be any hyperbolic complex, and denote $\mathcal{G}$ it 1 -skeleton with the path metric $d$. We will moreover extend the construction in [31] in two ways to provide a metric $\hat{d}=\hat{d}_{X}$ which is more general in the following sense:

- $\hat{d}$ is defined on all of $X$, rather than just on a discrete group $\Gamma$.

- $\hat{d}$ is invariant under the full isometry group $\operatorname{Isom}(X)$, rather than just under $\Gamma$.

- The $\operatorname{Isom}(X)$-action on $X$ is not assumed to be cocompact.

The construction in 31] utilizes a $\Gamma$-equivariant choice of geodesic paths $p[a, b]$ in the Cayley graph, viewed sometimes as a path and sometimes as a cellular 1-chain, from $a$ to $b$ for each pair $(a, b) \in \Gamma^{2}$. This bicombing $p[\cdot, \cdot]$ is not good enough for our purposes since in general it cannot be chosen to be $\operatorname{Isom}(X)-$ equivariant. This problem is fixed by using the 1-chain

$$
p^{\prime}[a, b]:=(\# \operatorname{Geod}(a, b))^{-1} \sum_{s \in \operatorname{Geod}(a, b)} s,
$$

where $\operatorname{Geod}(a, b)$ is the finite set of all geodesic paths in $\mathcal{G}$ from $a$ to $b$, instead of $p[a, b]$ whenever $p[a, b]$ was meant to be a 1-chain (and making no change 
when $p[a, b]$ was meant to be a path). Since $p^{\prime}[\cdot, \cdot]$ is $\operatorname{Isom}(X)$-equivariant, this provides a $\operatorname{Isom}(X)$-invariant metric $\hat{d}$ on $X^{(0)}$. The uniform local finiteness of $X$ guarantees that all the arguments of [31] go through with very minor modifications. (Namely, since the cardinality of balls of radius $7 \delta$ in $X^{(0)}$ might not be constant, though bounded above, one needs to change the definition of $\operatorname{star}(a)$ [31, page 100] to

$$
\operatorname{star}(a):=\frac{1}{\# B(a, 7 \delta)} \sum_{x \in B(a, 7 \delta)} x .
$$

Then the discussion of [31, page 111] should be changed as follows. Let $\omega_{\max }$ be the maximum of cardinalities of the balls of radius $7 \delta$ in $X^{(0)}$. Without loss of generality we can assume $\beta^{\prime} \leq \beta$, then

$$
\begin{aligned}
& \left|\operatorname{star}\left(f_{0}\right)+\alpha x_{0}-\operatorname{star}\left(f_{0}^{\prime}\right)-\alpha^{\prime} x_{0}\right|_{1} \\
& \leq\left|\operatorname{star}\left(f_{0}\right)+\alpha x_{0}-\beta x_{0}\right|_{1}+\left|-\operatorname{star}\left(f_{0}^{\prime}\right)-\alpha^{\prime} x_{0}+\beta^{\prime} x_{0}\right|_{1}+\left|\left(\beta-\beta^{\prime}\right) x_{0}\right|_{1} \\
& =(1-\beta)+\left(1-\beta^{\prime}\right)+\left(\beta-\beta^{\prime}\right)=2\left(1-\beta^{\prime}\right) \leq 2\left(1-\frac{1}{\omega_{\max }}\right) .
\end{aligned}
$$

The rest of the argument goes through.)

In particular, Theorem 26] still holds for this new metric $\hat{d}$ on $X^{(0)}$, and $\hat{d}$ is quasiisometric to $d$. Finally, let $\hat{d}=\hat{d}_{X}$ be the extension of $\hat{d}$ to all of $X$ by formula (31).

The hyperbolicity constant $\delta$ and the word-metric $d$ canonically depend on $X$, and $\hat{d}$ canonically depends on $\delta$ and $d$. This shows in particular that $\hat{d}$ is $\operatorname{Isom}(X)$-invariant. Recall that $\operatorname{Isom}(X)$ is the group of simplicial isometries of $(X, d)$.

Lemma 27 For a hyperbolic complex $X$ and a simplicial map $g: X \rightarrow X$, the following are equivalent.

(a) $g$ is a simplicial automorphism of $X$.

(b) $g$ is a simplicial automorphism of $X$ preserving the word metric $d$ on $X^{(1)}$.

(c) $g$ is a simplicial automorphism of $X$ preserving $\hat{d}$ on $X^{(0)}$.

(d) $g$ is a simplicial automorphism of $X$ preserving $\hat{d}$ on $X$.

In other words, there is no difference between simplicial isometries of $(X, d)$ and simplicial isometries of $(X, \hat{d})$. 


\subsection{Now use $\hat{d}$ instead of $d$ everywhere}

From now on we deal with hyperbolic complexes and we change the notations

of 1.1 redefining everything in terms of the metric $\hat{d}$ : the double difference $\langle\cdot, \cdot \mid \cdot, \cdot\rangle: X^{4} \rightarrow \mathbb{R}$ is

$$
\left\langle a, a^{\prime} \mid b, b^{\prime}\right\rangle:=\frac{1}{2}\left(\hat{d}(a, b)-\hat{d}\left(a^{\prime}, b\right)-\hat{d}\left(a, b^{\prime}\right)+\hat{d}\left(a^{\prime}, b^{\prime}\right)\right)
$$

and the Gromov product is

$$
\langle a \mid b\rangle_{c}:=\langle a, c \mid c, b\rangle=\frac{1}{2}(\hat{d}(a, c)+\hat{d}(b, c)-\hat{d}(a, b)) .
$$

With definition (32), Theorem [26] says that there exist $C \in[0, \infty)$ and $\mu \in$ $[0,1)$ such that for all $a, a^{\prime}, b, b^{\prime} \in X^{(0)}$ with $d\left(a, a^{\prime}\right) \leq 1$ and $d\left(b, b^{\prime}\right) \leq 1$, $\left|\left\langle a, a^{\prime} \mid b, b^{\prime}\right\rangle\right| \leq C \mu^{d(a, b)}$.

From now on the letter $d$ will always stand for the canonical word metric as in 5.1. Define the corresponding functions with respect to the word metric $d$ :

$$
\begin{aligned}
& \left(a, a^{\prime} \mid b, b^{\prime}\right):=\frac{1}{2}\left(d(a, b)-d\left(a^{\prime}, b\right)-d\left(a, b^{\prime}\right)+d\left(a^{\prime}, b^{\prime}\right)\right), \\
& (a \mid b)_{c}:=(a, c \mid c, b)=\frac{1}{2}(d(a, c)+d(b, c)-d(a, b)) .
\end{aligned}
$$

These functions can be partially extended to infinity; the extension is usually not continuous and is defined up to an additive constant.

Remark The linearity formula (31) implies that the double difference in $X$ is determined by its values on the vertices of $X$. Explicitly, if $a=\sum_{x} \alpha_{x} x$, $a^{\prime}=\sum_{x^{\prime}} \alpha_{x^{\prime}} x^{\prime}, b=\sum_{y} \beta_{y} y, b^{\prime}=\sum_{y^{\prime}} \beta_{y^{\prime}} y^{\prime}$ are convex combinations of vertices, then

$$
\left\langle a, a^{\prime} \mid b, b^{\prime}\right\rangle=\sum_{x} \sum_{x^{\prime}} \sum_{y} \sum_{y^{\prime}} \alpha_{x} \alpha_{x^{\prime}} \beta_{y} \beta_{y^{\prime}}\left\langle x, x^{\prime} \mid y, y^{\prime}\right\rangle
$$

Starting from the metric $\hat{d}$ (rather than from $d$ ) on $X$ we define $\ell, \beta^{\times}$, the pseudometric $d^{\times}$and the metric $d_{*}$ on $\oplus X$ as in 3.2 and 3.3, i.e. we denote $d_{*}:=\otimes \hat{d}\left(\right.$ rather than $\left.d_{*}:=\otimes d\right)$.

\subsection{Examples of hyperbolic complexes}

(1) As in $5.2(1)$, the Cayley graph of a hyperbolic group can be considered a hyperbolic complex with respect to the metric $\hat{d}_{\mathcal{G}}$. 
(2) The universal cover of a compact triangulated smooth manifold with hyperbolic fundamental group with respect to the metric $\Psi(d)$ as in $5.2(2)$, where $d$ is the intrinsic metric coming from the Riemannian structure.

(3) The universal cover of a compact triangulated manifold with hyperbolic fundamental group. One can take either the word metric $d$ or the canonical metric $\hat{d}$ defined in 6.1. both induced from the 0-skeleton (see 5.2 (3)). The two metrics are quasiisometric but $\hat{d}$ behaves better at infinity.

\subsection{Geodesics and nearest points}

"Geodesic" will always refer to the word metric $d$ in $\mathcal{G}=X^{(1)}$. Let $a, a^{\prime}, b, b^{\prime} \in$ $\overline{\mathcal{G}}, \alpha \in \operatorname{Geod}\left(a, a^{\prime}\right), \beta \in \operatorname{Geod}\left(b, b^{\prime}\right)$, so that $\alpha$ and $\beta$ are isometric embeddings of intervals: $\alpha: I \rightarrow \overline{\mathcal{G}}, \beta: J \rightarrow \overline{\mathcal{G}}$. Pick distance-minimizing vertices $a_{0} \in \alpha$ and $b_{0} \in \beta$, i.e. such that $d\left(a_{0}, b_{0}\right)=d(\alpha, \beta)$. Choose the parameterizing intervals $I$ and $J$ of $\alpha$ and $\beta$ containing 0 so that $\alpha(0)=a_{0}, \alpha(-M)=a$, $\alpha\left(M^{\prime}\right)=a^{\prime}, \beta(0)=b_{0}, \beta(-N)=b, \beta\left(N^{\prime}\right)=b^{\prime}$ for some non-negative (possibly infinite) $M, M^{\prime}, N, N^{\prime}$.

\section{Lemma 28}

(a) With the above notations, if $d(\alpha, \beta) \geq 2 \delta$, then for all $i \in I$ and $j \in J$,

$$
d(\alpha(i), \beta(j)) \geq|i|+d(\alpha, \beta)+|j|-6 \delta .
$$

In particular, $d(a, b) \geq d\left(a, a_{0}\right)+d\left(a_{0}, b_{0}\right)+d\left(b_{0}, b\right)-6 \delta$.

Now assume $a=a^{\prime}=a_{0}$, and $d\left(a_{0}, \beta\right)$ and $j \in J$ are arbitrary. Then

(b) $d\left(a_{0}, \beta(j)\right) \geq|j|+d\left(a_{0}, \beta\right)-2 \delta$,

(c) $\left.d\left(\left[a_{0}, b_{0}\right], \beta(j)\right]\right) \geq|j|-2 \delta$,

(d) $d\left(\left[a_{0}, b\right], \beta(j)\right) \geq j-3 \delta$.

Proof (a) From symmetry, it suffices to show the lemma when $i \geq 0$ and $j \geq 0$. Draw geodesics $[\alpha(i), \beta(j)]$ and $\left[a_{0}, \beta(j)\right]$, and inscribe triples of points in the triangles $\left\{a_{0}, \alpha(i), \beta(j)\right\}$ and $\left\{a_{0}, \beta(j), b_{0}\right\}$ as shown on Figure 2. Pick $v^{\prime} \in\left[a_{0}, b_{0}\right]$ with $d\left(a_{0}, v^{\prime}\right)=d\left(a_{0}, v_{1}\right)$. The vertex $v_{1}$ is $\delta$-close to $v_{2}$, and therefore to $\alpha$. If $d\left(a_{0}, v^{\prime}\right)>d\left(a_{0}, w_{1}\right)$ was true, then $v^{\prime}$ would be $\delta$-close to $b_{0}$, so $d(\alpha, \beta)<2 \delta$, which contradicts our assumption. Therefore $d\left(a_{0}, v^{\prime}\right) \leq d\left(a_{0}, w_{1}\right)$. It is an easy exercise to see from the figure that 


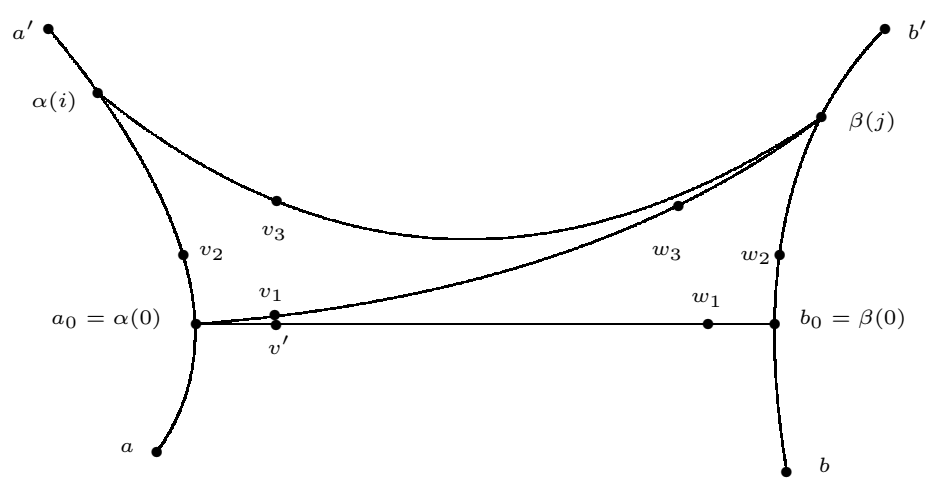

Figure 2: Illustration for Lemma 28

$d\left(b_{0}, w_{2}\right)=d\left(b_{0}, w_{1}\right) \leq \delta$ and $d\left(a_{0}, v_{1}\right)=d\left(a_{0}, v_{2}\right)=d\left(a_{0}, v^{\prime}\right) \leq 2 \delta$ (otherwise $\left[a_{0}, b_{0}\right]$ could be shortened), therefore

$$
\begin{aligned}
& d(\alpha(i), \beta(j))=d\left(\alpha(i), v_{2}\right)+d\left(v_{1}, \beta(j)\right) \\
& =d\left(\alpha(i), v_{2}\right)+d\left(v^{\prime}, w_{1}\right)+d\left(w_{2}, \beta(j)\right) \\
& \geq\left[d\left(\alpha(i), a_{0}\right)-2 \delta\right]+\left[d\left(a_{0}, b_{0}\right)-3 \delta\right]+\left[d\left(b_{0}, \beta(j)\right)-\delta\right] \\
& =i+d(\alpha, \beta)+j-6 \delta .
\end{aligned}
$$

(b) Since $a=a^{\prime}=a_{0}$, we also have $a=a^{\prime}=a_{0}=v_{1}=v_{2}=v_{3}=v^{\prime}$, and the inequality follows as in (a) by a similar, and simpler, argument.

(c) From symmetry it suffices to show the inequality in the case $j \geq 0$. If $j-2 \delta \leq 0$ then the inequality is obvious. Now suppose to the contrary that there exists $j>2 \delta$ and a point $x \in\left[a_{0}, b_{0}\right]$ such that $d(x, \beta(j))<j-2 \delta$. If $d\left(b_{0}, x\right) \leq d\left(b_{0}, w_{1}\right)$, then $d(x, \beta(j)) \geq d\left(b_{0}, \beta(j)\right)-d\left(b_{0}, x\right) \geq j-\delta$ which contradicts the assumption. Then $d\left(b_{0}, x\right) \geq d\left(b_{0}, w_{1}\right)$, so by the fine-triangles condition, there exists $y \in\left[a_{0}, \beta(j)\right]$ with

$$
d\left(a_{0}, y\right)=d\left(a_{0}, x\right) \leq d\left(a_{0}, w_{3}\right) \quad \text { and } \quad d(x, y) \leq \delta .
$$

Then

$$
d(x, \beta(j)) \geq d(y, \beta(j))-\delta \geq d\left(w_{3}, \beta(j)\right)-\delta=d\left(w_{2}, \beta(j)\right)-\delta \geq j-2 \delta
$$

provides a contradiction.

(d) If $j-3 \delta \leq 0$, then the inequality is obvious, so we assume $j>3 \delta$. From (c), $d\left(\left[a_{0}, b_{0}\right], \beta(j)\right) \geq|j|-2 \delta=j-2 \delta$, and since $\beta$ is geodesic, $d\left(\left[b_{0}, b\right], \beta(j)\right)=$ $|j|=j$. Since $\left[a_{0}, b\right]$ lies in the $\delta$-neighborhood of $\left[a_{0}, b_{0}\right] \cup\left[b_{0}, b\right]$,

$$
\begin{aligned}
& d\left(\left[a_{0}, b\right], \beta(j)\right) \geq d\left(\left[a_{0}, b_{0}\right] \cup\left[b_{0}, b\right], \beta(j)\right)-\delta \\
& \quad \geq \min \left\{d\left(\left[a_{0}, b_{0}\right], \beta(j)\right), d\left(\left[b_{0}, b^{\prime}\right], \beta(j)\right)\right\}-\delta \geq j-3 \delta .
\end{aligned}
$$


Given $y \in \overline{\mathcal{G}}$ and geodesics $\alpha$ and $\gamma$ in $\overline{\mathcal{G}}, \gamma$ is called a distance-minimizing geodesic from $y$ to $\alpha$ if $\gamma$ starts at $y$, terminates at a point $x \in \alpha$, and for every $z \in \gamma, d(z, x)=d(z, \alpha)$. A distance-minimizing geodesic exists for every pair $(y, \alpha)$. The set of all such terminal points $x$ over all distance-minimizing geodesics from $y$ to $\alpha$, denoted $\mathrm{np}[\alpha \mid y]$, is called the nearest point projection of $y$ to $\alpha$. When $\alpha=\left[a, a^{\prime}\right]$ we will use the notation $\operatorname{np}\left[a, a^{\prime} \mid y\right]$ for $\operatorname{np}[\alpha \mid y]$. For example, if $a \in \partial \mathcal{G}$ then $\{0\} \rightarrow\{a\}$ is the only distance-minimizing geodesic from $a$ to $\left[a, a^{\prime}\right]$, so $\operatorname{np}\left[a, a^{\prime} \mid a\right]=\{a\}$.

Given two geodesics $\alpha$ and $\beta$ in $\overline{\mathcal{G}}, \gamma$ is called a distance-minimizing geodesic from $\alpha$ to $\beta$ if $\gamma$ is a geodesic in $\overline{\mathcal{G}}, \gamma$ starts at a point $x \in \alpha$, terminates at a point $y \in \beta$, and for every $z \in \gamma, d(z, x)=d(z, \alpha)$ and $d(z, y)=d(z, \beta)$. Such a pair $(x, y) \in \alpha \times \beta$ will be called a distance-minimizing pair for $(\alpha, \beta)$. Every pair $(\alpha, \beta)$ of geodesics in $\overline{\mathcal{G}}$ admits a distance-minimizing pair.

Lemma 29 Let $b, b^{\prime} \in \overline{\mathcal{G}}, \beta \in \operatorname{Geod}\left(b, b^{\prime}\right), x, y \in \mathcal{G}, x^{\prime} \in \mathrm{np}[\beta \mid x], y^{\prime} \in \mathrm{np}[\beta \mid y]$ (see Figure 3).

(a) If $d\left(x^{\prime}, y^{\prime}\right) \geq 2 \delta$, then $d(x, y) \geq d\left(x, x^{\prime}\right)+d\left(x^{\prime}, y^{\prime}\right)+d\left(y^{\prime}, y\right)-6 \delta$.

(b) For any positive integer $n$, if $d(x, y)<n \delta$, then $d\left(x^{\prime}, y^{\prime}\right)<(n+6) \delta$.

(c) Take any $\alpha \in \operatorname{Geod}(x, y)$ and let $\beta^{\prime}$ be the part of $\beta$ between $x^{\prime}$ and $y^{\prime}$. Then for any $z \in \alpha$ and $z^{\prime} \in \operatorname{np}[\beta \mid z], d\left(\beta^{\prime}, z^{\prime}\right) \leq 8 \delta$.

(1)

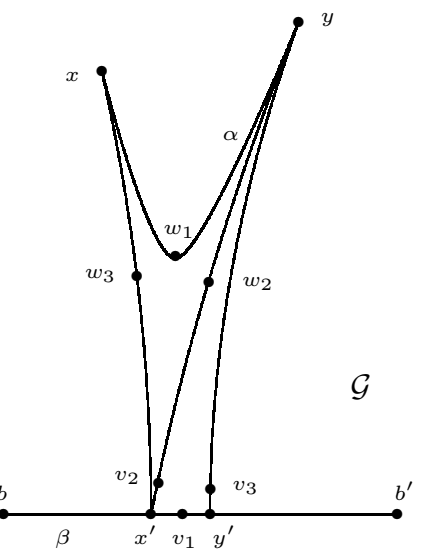

(2)

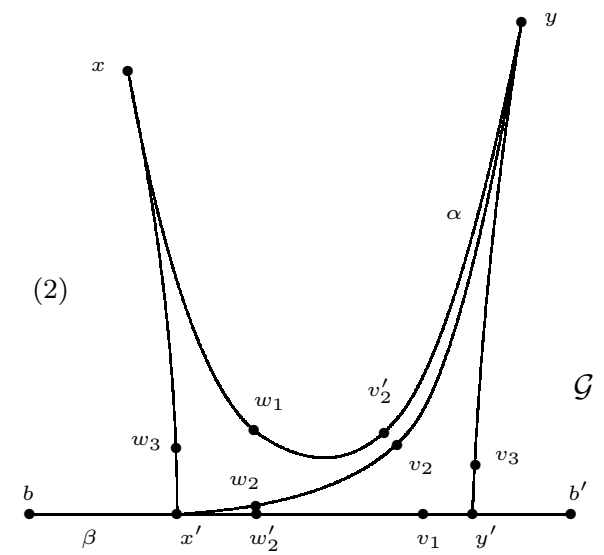

Figure 3: Illustration for Lemma 29]

Proof (a) Set the notations as in Figure 31(2). If $d\left(x^{\prime}, v_{1}\right)<d\left(x^{\prime}, w_{2}^{\prime}\right)$, then $w_{2}^{\prime}$ would be $\delta$-close to $y^{\prime}$, so $d\left(x^{\prime}, y^{\prime}\right)<2 \delta$, which contradicts our assumption. 
So $d\left(x^{\prime}, w_{2}^{\prime}\right) \leq d\left(x^{\prime}, v_{1}\right)$. We have

$$
d\left(x^{\prime}, w_{2}\right)=d\left(x^{\prime}, w_{3}\right)=d\left(x^{\prime}, w_{2}^{\prime}\right) \leq 2 \delta \quad \text { and } \quad d\left(y^{\prime}, v_{3}\right)=d\left(y^{\prime}, v_{1}\right) \leq \delta
$$

(otherwise $\left[x, x^{\prime}\right]$ or $\left[y, y^{\prime}\right]$ could be shortened), hence

$$
\begin{aligned}
& d(x, y)=d\left(x, w_{3}\right)+d\left(w_{2}, y\right)=d\left(x, w_{3}\right)+d\left(w_{2}^{\prime}, v_{1}\right)+d\left(v_{3}, y\right) \\
& \geq\left[d\left(x, x^{\prime}\right)-2 \delta\right]+\left[d\left(x^{\prime}, y^{\prime}\right)-3 \delta\right]+\left[d\left(y^{\prime}, y\right)-\delta\right] \\
& =d\left(x, x^{\prime}\right)+d\left(x^{\prime}, y^{\prime}\right)+d\left(y^{\prime}, y\right)-6 \delta .
\end{aligned}
$$

(b) The proof is by contradiction: if $d\left(x^{\prime}, y^{\prime}\right) \geq(n+6) \delta$, then $d\left(x^{\prime}, y^{\prime}\right) \geq 2 \delta$ hence by (a), $d(x, y) \geq d\left(x, x^{\prime}\right)+d\left(x^{\prime}, y^{\prime}\right)+d\left(y^{\prime}, y\right)-6 \delta \geq d\left(x^{\prime}, y^{\prime}\right)-6 \delta \geq n \delta$.

(c) Draw the geodesic $\left[x^{\prime}, y\right]$ and inscribe triples of points in the triangles $\left\{x, y, x^{\prime}\right\}$ and $\left\{y, x^{\prime}, y^{\prime}\right\}$. There are two cases as on Figure 3 depending on the order of $v_{2}$ and $w_{2}$ along $\left[x^{\prime}, y\right]$. Using the $\delta$-fine property it is easy to see from the figure that in either case $\alpha$ lies in the $2 \delta$-neighborhood of $\left[x, x^{\prime}\right] \cup \beta^{\prime} \cup\left[y^{\prime}, y\right]$. Pick any $z \in \alpha$ and $z^{\prime} \in \operatorname{np}[\beta \mid z]$, then there exists $w \in\left[x, x^{\prime}\right] \cup \beta^{\prime} \cup\left[y^{\prime}, y\right]$ with $d(z, w) \leq 2 \delta$. But for any $w \in\left[x, x^{\prime}\right] \cup \beta^{\prime} \cup\left[y^{\prime}, y\right]$, there is a projection $w^{\prime} \in$ $\mathrm{np}[\beta \mid w]$ which also belongs to $\beta^{\prime}$. By (b) applied to $z$ and $w, d\left(z^{\prime}, w^{\prime}\right) \leq 8 \delta$, so $d\left(\beta^{\prime}, z^{\prime}\right) \leq 8 \delta$.

\subsection{Neighborhoods in $\overline{\mathcal{G}}$ and $\bar{X}$}

As before, $X$ will be a hyperbolic complex, and $\mathcal{G}:=X^{(1)}$ with the word metric $d$. We present a description of the topology on $\bar{X}=X \cup \partial X$ in terms of half-spaces. The idea of this presentation is due to Cannon, and the details were written out by Swenson [37. It was proved in 37] that half-spaces generate the same topology as the one introduced by Gromov [27; see also [8] for a list of properties.

Pick a basepoint $x_{0} \in \mathcal{G}^{(0)}$. Given $a \in \partial \mathcal{G}$ and $t \in \mathbb{R}$, denote

$$
\begin{aligned}
& U^{+}(a, t):=\left\{x \in \overline{\mathcal{G}} \mid \exists x^{\prime} \in \operatorname{np}\left[x_{0}, a \mid x\right] \quad d\left(x_{0}, x^{\prime}\right) \geq t\right\}, \\
& U^{-}(a, t):=\left\{x \in \overline{\mathcal{G}} \mid \exists x^{\prime} \in \operatorname{np}\left[x_{0}, a \mid x\right] \quad d\left(x_{0}, x^{\prime}\right) \leq t\right\} .
\end{aligned}
$$

Now let $V^{ \pm}(a, t):=U^{ \pm}(a, t)$ be the union of simplices in $X$ whose vertices lie in $U^{ \pm}(a, t)$. The sets $V^{+}(a, t) \subseteq \bar{X}$ form a fundamental system of closed neighborhoods of $a \in \partial X$ in $\bar{X}$.

Lemma 30 For any $a \in \partial \mathcal{G}$ and any $s, t \in \mathbb{R}$,

$$
d\left(U^{-}(a, s), U^{+}(a, t)\right) \geq t-s-6 \delta .
$$


Proof If $t<s+6 \delta$ then the inequality is obvious. Now pick arbitrary

$$
s \in \mathbb{R}, \quad t \in[s+6 \delta, \infty), \quad x \in U^{-}(a, s), \quad y \in U^{+}(a, t) .
$$

By the definition of $U^{ \pm}$, we can choose $x^{\prime} \in \mathrm{np}\left[x_{0}, a \mid x\right]$ and $y^{\prime} \in \mathrm{np}\left[x_{0}, a \mid y\right]$ so that $d\left(x_{0}, x^{\prime}\right) \leq s$ and $d\left(x_{0}, y^{\prime}\right) \geq t$. Since $d\left(x^{\prime}, y^{\prime}\right) \geq t-s \geq 6 \delta$, using Lemma 29, $d(x, y) \geq d\left(x^{\prime}, y^{\prime}\right)-6 \delta \geq t-s-6 \delta$.

For $S_{1}, S_{2} \subseteq \overline{\mathcal{G}},\left[S_{1}, S_{2}\right]$ will denote the union of the images of all geodesics in $\overline{\mathcal{G}}$ connecting points of $S_{1}$ to points in $S_{2}$.

Lemma 31 Let $a \in \partial \mathcal{G}$ and $s \in[0, \infty)$, then

$$
\left[U^{+}(a, s), U^{+}(a, s)\right] \subseteq U^{+}(a, s-8 \delta) \text { and }\left[U^{-}(a, s), U^{-}(a, s)\right] \subseteq U^{-}(a, s+8 \delta) \text {. }
$$

Proof Pick any $x, y \in U^{+}(a, s), \alpha \in \operatorname{Geod}(x, y)$. By the definition of $U^{+}$, there exist projections $x^{\prime} \in \mathrm{np}\left[x_{0}, a \mid x\right]$ and $y^{\prime} \in \mathrm{np}\left[x_{0}, a \mid y\right]$ with $d\left(x_{0}, x^{\prime}\right) \geq s$ and $d\left(x_{0}, y^{\prime}\right) \geq s$. Let $\beta^{\prime}$ be the interval in $\left[x_{0}, a\right]$ between $x^{\prime}$ and $y^{\prime}$. By Lemma 29] (c), the projection of $\alpha$ into $\left[x_{0}, a\right]$ lies in the $8 \delta$-neighborhood of $\beta^{\prime}$, so $\alpha \subseteq U^{+}(a, s-8 \delta)$. The second inclusion is treated similarly.

\subsection{Properties of $\hat{d}$}

Theorem 32 For any hyperbolic complex $X$ there is a canonically associated metric $\hat{d}=\hat{d}_{X}$ with the following properties.

(a) $\hat{d}$ is quasiisometric to the word metric $d_{X}$, i.e there exist $A \in[1, \infty)$ and $B \in[0, \infty)$ depending only on $X$ such that for all $x, y \in X$,

$$
d(x, y) / A-B \leq \hat{d}(x, y) \leq A d(x, y)+B .
$$

(b) $\hat{d}$ is $\operatorname{Isom}(X)$-invariant: $\hat{d}(g x, g y)=\hat{d}(x, y)$ for $g \in \operatorname{Isom}(X)$.

(c) $(X, \hat{d})$ is a metric complex, that is $\hat{d}$ is induced from the 0 -skeleton.

(d) There exist constants $L \in[0, \infty)$ and $\mu \in[0,1)$ depending only on $X$ with the following property. If $a, a^{\prime}, b, b^{\prime} \in X^{(0)}, \alpha \in \operatorname{Geod}\left(a, a^{\prime}\right), \beta \in$ $\operatorname{Geod}\left(b, b^{\prime}\right)$ and $d(\alpha, \beta) \geq 2 \delta$, then $\left|\left\langle a, a^{\prime} \mid b, b^{\prime}\right\rangle\right| \leq L \mu^{d(\alpha, \beta)}$.

Proof Take the metric $\hat{d}$ as described in 6.1. (a) and (b) are shown in theorems 17 and 6(2) in 31] for $\mathcal{G}$, this easily implies (a) and (b) for the whole $X$. (c) holds by definition. It only remains to show (d). Pick arbitrary vertices $a, a^{\prime}, b, b^{\prime}$ satisfying the hypotheses. Set notations as in Figure 2 pick a 
distance-minimizing pair $\left(a_{0}, b_{0}\right)$ for $(\alpha, \beta)$ and choose parametrizations of $\alpha$ and $\beta$ such that $a=\alpha(-M), a^{\prime}=\alpha\left(M^{\prime}\right), b=\beta(-N), b^{\prime}=\beta\left(N^{\prime}\right)$ for some non-negative integers $M, M^{\prime}, N, N^{\prime}$. Then by Lemma 28 and Theorem 26 . taking the sums of geometric series,

$$
\begin{aligned}
& \left|\left\langle a, a^{\prime} \mid b, b^{\prime}\right\rangle\right|=\left|\sum_{i=-M}^{M^{\prime}-1} \sum_{j=-N}^{N^{\prime}-1}\langle\alpha(i), \alpha(i+1) \mid \beta(j), \beta(j+1)\rangle\right| \\
& \leq \sum_{i=-M}^{M^{\prime}-1} \sum_{j=-N}^{N^{\prime}-1} C \mu^{d(\alpha(i), \beta(j))} \leq \sum_{i=-\infty}^{\infty} \sum_{j=-\infty}^{\infty} C \mu^{|i|+|j|+d(\alpha, \beta)-6 \delta} \\
& \leq 4 \frac{C \mu^{d(\alpha, \beta)-6 \delta}}{(1-\mu)^{2}}=L \mu^{d(\alpha, \beta)},
\end{aligned}
$$

where we denoted $L:=4 C \mu^{-6 \delta} /(1-\mu)^{2}$.

Proposition 33 Let $\mathcal{G}$ be a hyperbolic graph of bounded valence. There exists $C^{\prime} \in[0, \infty)$ depending only on $\mathcal{G}$ such that if $u, v, w \in \mathcal{G}^{(0)}$ and $w$ lies on a geodesic in $\mathcal{G}$ connecting $u$ to $v$, then $|\hat{d}(u, v)-\hat{d}(u, w)-\hat{d}(w, v)| \leq C^{\prime}$.

This immediately follows from the definition of $\hat{d}$ and Proposition 10(b) in [31. The proposition says in other words that geodesics in $(\mathcal{G}, d)$ can be parameterized to become ${ }^{+}$geodesic in $(\mathcal{G}, \hat{d})$, that is geodesic up to an additive constant, where the constant depends only on $\mathcal{G}$. Note though that $d$ and $\hat{d}$ are not necessarily ${ }^{+}$equivalent.

Lemma 34 There exists $P \in[0, \infty)$ depending only on $X$ with the following property. Let $a_{0} \in X^{(0)}, b, b^{\prime} \in X^{(0)} \cup \partial X, \beta \in \operatorname{Geod}\left(b, b^{\prime}\right)$ and $b_{0} \in \mathrm{np}\left[\beta \mid a_{0}\right]$. If $b, b^{\prime} \in \partial X$, assume $b \neq b^{\prime}$. Then $\left|\left\langle b \mid b^{\prime}\right\rangle_{a_{0}}-\hat{d}\left(a_{0}, b_{0}\right)\right| \leq P$.

Note that the statement is about $\hat{d}$, but nearest points are defined using $d$.

Proof Assume first that $a, b, b^{\prime} \in X^{(0)}$. We use hyperbolicity and Proposition 33 .

$$
\begin{aligned}
& \left\langle b \mid b^{\prime}\right\rangle_{a_{0}}-\hat{d}\left(a_{0}, b_{0}\right) \simeq \frac{1}{2}\left(\hat{d}\left(a_{0}, b\right)+\hat{d}\left(a_{0}, b^{\prime}\right)-\hat{d}\left(b, b^{\prime}\right)\right)-\hat{d}\left(a_{0}, b_{0}\right) \\
& \simeq \frac{1}{2}\left(\hat{d}\left(a_{0}, b_{0}\right)+\hat{d}\left(b_{0}, b\right)+\hat{d}\left(a_{0}, b_{0}\right)+\hat{d}\left(b_{0}, b^{\prime}\right)-\hat{d}\left(b, b^{\prime}\right)\right)-\hat{d}\left(a_{0}, b_{0}\right) \\
& =\frac{1}{2}\left(\hat{d}\left(b_{0}, b\right)+\hat{d}\left(b_{0}, b^{\prime}\right)-\hat{d}\left(b, b^{\prime}\right)\right) \simeq 0,
\end{aligned}
$$

where $\widetilde{f}$ is ${ }^{+}$equivalence with a constant that depends only on $X$. This extends to the case when $b$ or $b^{\prime}$ is in $\partial X$. 


\subsection{Extension of double difference}

Let $\left(a, a^{\prime}, b, b^{\prime}\right) \in \bar{X}^{4}$. A $\partial X$-triple in $\left(a, a^{\prime}, b, b^{\prime}\right)$ is a set of three distinct letters taken from $a, a^{\prime}, b, b^{\prime}$ such that each letter represents a point in $\partial X \subseteq \bar{X}$. A $\partial X$-triple is trivial if the three letters represent the same point in $\partial X$. Denote

$$
\bar{X}^{\diamond}:=\left\{\left(a, a^{\prime}, b, b^{\prime}\right) \in \bar{X}^{4} \mid \text { each } \partial X \text {-triple in }\left(a, a^{\prime}, b, b^{\prime}\right) \text { is non-trivial }\right\} .
$$

We have $X^{4} \subseteq \bar{X}^{\diamond} \subseteq \bar{X}^{4}$. The topology on $\bar{X}^{\diamond}$ is induced by the last inclusion, and $X^{4}$ is dense in both $\bar{X}^{\diamond}$ and $\bar{X}^{4}$.

Recall that $\overline{\mathbb{R}}:=[-\infty, \infty]$ is the two-point compactification of $\mathbb{R}$.

Theorem 35 Suppose $X$ is a hyperbolic complex, then the double difference $\langle\cdot, \cdot \mid \cdot, \cdot\rangle: X^{4} \rightarrow \mathbb{R}$ with respect to $\hat{d}$ defined in (32) extends to a continuous Isom $(X)$-invariant function $\langle\cdot, \cdot \mid \cdot, \cdot\rangle: \bar{X}^{\diamond} \rightarrow \overline{\mathbb{R}}$ with the following properties.

(a) $\left\langle a, a^{\prime} \mid b, b^{\prime}\right\rangle=\left\langle b, b^{\prime} \mid a, a^{\prime}\right\rangle$.

(b) $\left\langle a, a^{\prime} \mid b, b^{\prime}\right\rangle=-\left\langle a^{\prime}, a \mid b, b^{\prime}\right\rangle=-\left\langle a, a^{\prime} \mid b^{\prime}, b\right\rangle$.

(c) $\left\langle a, a \mid b, b^{\prime}\right\rangle=0,\left\langle a, a^{\prime} \mid b, b\right\rangle=0$.

(d) $\left\langle a, a^{\prime} \mid b, b^{\prime}\right\rangle+\left\langle a^{\prime}, a^{\prime \prime} \mid b, b^{\prime}\right\rangle=\left\langle a, a^{\prime \prime} \mid b, b^{\prime}\right\rangle$, where by convention we allow $\pm \infty \mp \infty=r$ and $\pm \infty+r= \pm \infty$ for any $r \in \mathbb{R}$, and $\pm \infty \pm \infty= \pm \infty$.

(e) $\langle a, b \mid c, x\rangle+\langle b, c \mid a, x\rangle+\langle c, a \mid b, x\rangle=0$ with the same convention.

(f) $\left\langle a, a^{\prime} \mid b, b^{\prime}\right\rangle=\infty$ if and only if $a=b^{\prime} \in \partial X$ or $a^{\prime}=b \in \partial X$.

(g) $\left\langle a, a^{\prime} \mid b, b^{\prime}\right\rangle=-\infty$ if and only if $a=b \in \partial X$ or $a^{\prime}=b^{\prime} \in \partial X$.

(h) Let $(\cdot, \cdot \cdot, \cdot)$ be the double difference with respect to the word metric $d$ as in (34). Then $\langle\cdot, \cdot \mid \cdot, \cdot\rangle$ and $(\cdot, \cdot \mid \cdot, \cdot)$ are ${ }^{\times+}$equivalent as functions on $\bar{X}^{\diamond}$.

Proof For $\left(a, a^{\prime}, b, b^{\prime}\right) \in \bar{X}^{4}$, the pairs of letters $(a, b),\left(a^{\prime}, b^{\prime}\right),\left(a, b^{\prime}\right),\left(a^{\prime}, b\right)$ and their inverses will be called side pairs. A side $\partial X$-pair is a side pair in which each letter represents a point in $\partial X$. A side $\partial X$-pair is trivial if the two letters represent the same point. Consider the intermediate set

$$
S:=\left\{\left(a, a^{\prime}, b, b^{\prime}\right) \in \bar{X}^{4} \mid \text { each side } \partial \mathrm{X} \text {-pair in }\left(a, a^{\prime}, b, b^{\prime}\right) \text { is non-trivial }\right\} ;
$$

one checks that $X^{4} \subseteq S \subseteq \bar{X}^{\diamond}$.

We first extend $\langle\cdot, \cdot \mid \cdot, \cdot\rangle$ to a continuous function $S \rightarrow \mathbb{R}$. Since $X^{4}$ is dense in $S$ and $\mathbb{R}$ is regular, it suffices to prove the existence of

$$
\lim \left\langle a, a^{\prime} \mid b, b^{\prime}\right\rangle \quad \text { as } \quad\left(a, a^{\prime}, b, b^{\prime}\right) \rightarrow\left(\bar{a}, \bar{a}^{\prime}, \bar{b}, \bar{b}^{\prime}\right) \text { along } X^{4}
$$


in $\mathbb{R}$ for each $\left(\bar{a}, \bar{a}^{\prime}, \bar{b}, \bar{b}^{\prime}\right) \in S \backslash X^{4}$ (see [1, I $\S 8 \mathrm{~N}^{\circ} 5$, Theorem 1]). In each such $\left(\bar{a}, \bar{a}^{\prime}, \bar{b}, \bar{b}^{\prime}\right)$ at least one of $\bar{a}, \bar{a}^{\prime}, \bar{b}, \bar{b}^{\prime}$ is in $\partial X$ and there are no trivial side $\partial X$-pairs. We will present the proof in the case when $\bar{a}, \bar{a}^{\prime}, \bar{b}, \bar{b}^{\prime}$ are all in $\partial X$; the other cases are similar.

Since $\overline{\mathcal{G}}$ is Hausdorff, there is a number $s \in \mathbb{R}$ such that

$$
\begin{aligned}
& U^{+}(\bar{a}, s) \cap U^{+}\left(\bar{b}^{\prime}, s\right)=U^{+}\left(\bar{b}^{\prime}, s\right) \cap U^{+}\left(\bar{a}^{\prime}, s\right) \\
& =U^{+}\left(\bar{a}^{\prime}, s\right) \cap U^{+}(\bar{b}, s)=U^{+}(\bar{b}, s) \cap U^{+}(\bar{a}, s)=\emptyset,
\end{aligned}
$$

So in particular $U^{+}(\bar{b}, s) \cup U^{+}\left(\bar{b}^{\prime}, s\right) \subseteq U^{-}(\bar{a}, s)$, and Lemma 31 implies

$$
\left[U^{+}(\bar{b}, s+8 \delta), U^{+}\left(\bar{b}^{\prime}, s+8 \delta\right)\right] \subseteq\left[U^{-}(\bar{a}, s), U^{-}(\bar{a}, s)\right] \subseteq U^{-}(\bar{a}, s+8 \delta),
$$

hence replacing $s$ with $s+6 \delta$ we can assume $\left[U^{+}(\bar{b}, s), U^{+}\left(\bar{b}^{\prime}, s\right)\right] \subseteq U^{-}(\bar{a}, s)$. The same argument applies to the cyclic permutations of $\left(\bar{a}, \bar{b}, \bar{a}^{\prime}, \bar{b}^{\prime}\right)$. Denote

$$
\alpha:=\left[x_{0}, \bar{a}\right], \quad \alpha^{\prime}:=\left[x_{0}, \bar{a}^{\prime}\right], \quad \beta:=\left[x_{0}, \bar{b}\right], \quad \beta^{\prime}:=\left[x_{0}, \bar{b}^{\prime}\right] .
$$

Pick any $i \geq s+8 \delta$. We have $\alpha(i), \alpha(i+1) \in U^{+}(\bar{a}, i)$, therefore the edge $[\alpha(i), \alpha(i+1)]$ also lies in $U^{+}(\bar{a}, i)$. Also $\beta(i) \in U^{+}(\bar{b}, i) \subseteq U^{+}(\bar{b}, i) \subseteq U^{+}(\bar{b}, s)$ and similarly $\beta^{\prime}(i+1) \in U^{+}\left(\bar{b}^{\prime}, s\right)$, hence $\left[\beta(i), \beta^{\prime}(i+1)\right] \subseteq\left[U^{+}(\bar{b}, s), U^{+}\left(\bar{b}^{\prime}, s\right)\right] \subseteq$ $U^{-}(\bar{a}, s)$ (see Figure 4). By Lemma 30 .

$$
d\left(\left[\beta(i), \beta^{\prime}(i+1)\right],[\alpha(i+1), \alpha(i)]\right) \geq d\left(U^{-}(\bar{a}, s), U^{+}(\bar{a}, i)\right) \geq i-s-6 \delta \geq 2 \delta .
$$

hence by Theorem $32(\mathrm{~d})$,

$$
\left|\left\langle\alpha(i+1), \alpha(i) \mid \beta(i), \beta^{\prime}(i+1)\right\rangle\right| \leq L \mu^{d\left(\left[\beta(i), \beta^{\prime}(i+1)\right],[\alpha(i+1), \alpha(i)]\right)} \leq L \mu^{i-s-6 \delta},
$$

and similarly for the cyclic permutations of $\left(\alpha, \beta, \alpha^{\prime}, \beta^{\prime}\right)$; then

$$
\begin{aligned}
& \left|\left\langle\alpha(i+1), \alpha^{\prime}(i+1) \mid \beta(i+1), \beta^{\prime}(i+1)\right\rangle-\left\langle\alpha(i), \alpha^{\prime}(i) \mid \beta(i), \beta^{\prime}(i)\right\rangle\right| \\
& =\mid\left\langle\alpha(i+1), \alpha(i) \mid \beta(i), \beta^{\prime}(i+1)\right\rangle-\left\langle\beta(i+1), \beta(i) \mid \alpha^{\prime}(i), \alpha(i+1)\right\rangle \\
& +\left\langle\alpha^{\prime}(i+1), \alpha^{\prime}(i) \mid \beta^{\prime}(i), \beta(i+1)\right\rangle-\left\langle\beta^{\prime}(i+1), \beta^{\prime}(i) \mid \alpha(i), \alpha^{\prime}(i+1)\right\rangle \mid \\
& \leq 4 L \mu^{i-s-6 \delta}
\end{aligned}
$$

and

$$
\begin{aligned}
& \sum_{i=s}^{\infty}\left|\left\langle\alpha(i+1), \alpha^{\prime}(i+1) \mid \beta(i+1), \beta^{\prime}(i+1)\right\rangle-\left\langle\alpha(i), \alpha^{\prime}(i) \mid \beta(i), \beta^{\prime}(i)\right\rangle\right| \\
& \leq \sum_{i=s}^{\infty} 4 L \mu^{i-s-6 \delta}=\frac{4 L \mu^{-6 \delta}}{1-\mu} .
\end{aligned}
$$

This shows that $\left\langle\alpha(i), \alpha^{\prime}(i) \mid \beta(i), \beta^{\prime}(i)\right\rangle$ is a Cauchy sequence in $\mathbb{R}$ so it has a limit in $\mathbb{R}$ which we denote $\left\langle\bar{a}, \bar{a}^{\prime} \mid \bar{b}, \bar{b}^{\prime}\right\rangle$. We show that $\left\langle\bar{a}, \bar{a}^{\prime} \mid \bar{b}, \bar{b}^{\prime}\right\rangle$ is indeed the 


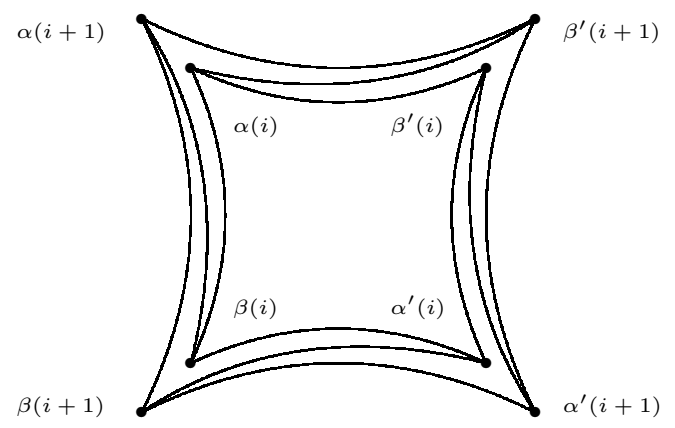

Figure 4: Extending $\langle\cdot, \cdot \mid \cdot, \cdot\rangle$ from $X^{4}$ to $S$

limit in (36). For any $i \geq s+16 \delta$ and any $\left(a, a^{\prime}, b, b^{\prime}\right) \in\left(U^{+}(\bar{a}, i) \times U^{+}\left(\bar{a}^{\prime}, i\right) \times\right.$ $\left.U^{+}(\bar{b}, i) \times U^{+}\left(\bar{b}^{\prime}, i\right)\right) \cap\left(X^{(0)}\right)^{4}$, we have by lemmas 31 and 30 ,

$$
\begin{aligned}
d\left(\left[\beta(i), b^{\prime}\right],[a, \alpha(i)]\right) & \geq d\left(U^{-}(\bar{a}, s), U^{+}(\bar{a}, i-8 \delta)\right) \\
& \geq(i-8 \delta)-s-6 \delta=i-s-14 \delta \geq 2 \delta
\end{aligned}
$$

and the same for the cyclic permutations of $\left(\alpha, \alpha^{\prime}, \beta, \beta^{\prime}\right)$. Then similarly to Figure 4

$$
\begin{aligned}
& \quad\left|\left\langle a, a^{\prime} \mid b, b^{\prime}\right\rangle-\left\langle\alpha(i), \alpha^{\prime}(i) \mid \beta(i), \beta^{\prime}(i)\right\rangle\right| \\
& =\mid\left\langle a, \alpha(i) \mid \beta(i), b^{\prime}\right\rangle-\left\langle b, \beta(i) \mid \alpha^{\prime}(i), a\right\rangle \\
& \quad+\left\langle a^{\prime}, \alpha^{\prime}(i) \mid \beta^{\prime}(i), b\right\rangle-\left\langle b^{\prime}, \beta^{\prime}(i) \mid \alpha(i), a^{\prime}\right\rangle \mid \\
& \leq 4 L \mu^{i-s-14 \delta} \underset{i \rightarrow \infty}{\rightarrow} 0 .
\end{aligned}
$$

The above inequality is proved for vertices $a, a^{\prime}, b, b^{\prime}$, but by linearity of double difference over simplices (35) it also holds in the case when

$$
\left(a, a^{\prime}, b, b^{\prime}\right) \in\left(V^{+}(\bar{a}, i) \times V^{+}\left(\bar{a}^{\prime}, i\right) \times V^{+}(\bar{b}, i) \times V^{+}\left(\bar{b}^{\prime}, i\right)\right) \cap X^{4} .
$$

This implies that $\left\langle\bar{a}, \bar{a}^{\prime} \mid \bar{b}, \bar{b}^{\prime}\right\rangle$ is the limit in (36).

Now we want to extend $\langle\cdot, \cdot \mid \cdot, \cdot\rangle$ to a continuous function $\bar{X}^{\diamond} \rightarrow \overline{\mathbb{R}}$. Pick an arbitrary $\left(\bar{a}, \bar{a}^{\prime}, \bar{b}, \bar{b}^{\prime}\right) \in \bar{X}^{\diamond} \backslash S$. This means that there is a trivial side $\partial X$-pair in $\left(\bar{a}, \bar{a}^{\prime}, \bar{b}, \bar{b}^{\prime}\right)$, for example $\bar{a}=\bar{b}^{\prime} \in \partial X$, then $\bar{a}^{\prime} \neq \bar{a}=\bar{b}^{\prime} \neq \bar{b}$. Again, since $S$ is dense in $\bar{X}^{\diamond}$ and $\overline{\mathbb{R}}$ is regular, it suffices to prove the existence of

$$
\lim \left\langle a, a^{\prime} \mid b, b^{\prime}\right\rangle \quad \text { as } \quad\left(a, a^{\prime}, b, b^{\prime}\right) \rightarrow\left(\bar{a}, \bar{a}^{\prime}, \bar{b}, \bar{b}^{\prime}\right) \text { along } S
$$

in $\overline{\mathbb{R}}$. Fix any $a_{0} \in X^{(0)}$, then we have $\left(\bar{a}, \bar{a}^{\prime}, \bar{b}, a_{0}\right),\left(a_{0}, \bar{a}^{\prime}, a_{0}, \bar{b}^{\prime}\right) \in S$. Above 
we proved that $\langle\cdot, \cdot \mid \cdot, \cdot\rangle$ is continuous on $S$ and takes values in $\mathbb{R}$, hence

$$
\begin{gathered}
\lim \left\langle a, a^{\prime} \mid b, a_{0}\right\rangle=\left\langle\bar{a}, \bar{a}^{\prime} \mid \bar{b}, a_{0}\right\rangle \in \mathbb{R} \quad \text { and } \\
\lim \left\langle a_{0}, a^{\prime} \mid a_{0}, b^{\prime}\right\rangle=\left\langle a_{0}, \bar{a}^{\prime} \mid a_{0}, \bar{b}^{\prime}\right\rangle \in \mathbb{R} \\
\text { as }\left(a, a^{\prime}, b, b^{\prime}\right) \rightarrow\left(\bar{a}, \bar{a}^{\prime}, \bar{b}, \bar{b}^{\prime}\right) \text { along } S . \\
\left\langle a, a^{\prime} \mid b, b^{\prime}\right\rangle=\left\langle a, a^{\prime} \mid b, a_{0}\right\rangle+\left\langle a, a^{\prime} \mid a_{0}, b^{\prime}\right\rangle \\
=\left\langle a, a^{\prime} \mid b, a_{0}\right\rangle+\left\langle a, a_{0} \mid a_{0}, b^{\prime}\right\rangle+\left\langle a_{0}, a^{\prime} \mid a_{0}, b^{\prime}\right\rangle
\end{gathered}
$$

holds if all the terms are in $X^{4}$ and therefore, by continuity, in $S$. Pick any $i \in \mathbb{R}$ and $\left(a, a^{\prime}, b, b^{\prime}\right) \in\left(U^{+}(\bar{a}, i) \times U^{+}\left(\bar{a}^{\prime}, i\right) \times U^{+}(\bar{b}, i) \times U^{+}\left(\bar{b}^{\prime}, i\right)\right) \cap S$. Let $b_{0} \in \mathrm{np}\left[a, b^{\prime} \mid a_{0}\right]$. It follows from Lemma 30 that $d\left(a_{0}, U^{+}(\bar{a}, i-6 \delta)\right) \rightarrow \infty$ as $i \rightarrow \infty$. Since $d$ and $\hat{d}$ are quasiisometric (Theorem [32), the same holds for $\hat{d}\left(a_{0}, U^{+}(\bar{a}, i-6 \delta)\right)$. By Lemma 31] $\left[a, b^{\prime}\right] \subseteq\left[U^{+}(\bar{a}, i), U^{+}(\bar{a}, i)\right] \subseteq U^{+}(\bar{a}, i-8 \delta)$, then by Lemma 34

$$
\begin{aligned}
& \left\langle a, a_{0} \mid a_{0}, b^{\prime}\right\rangle=\left\langle a \mid b^{\prime}\right\rangle_{a_{0}} \geq \hat{d}\left(a_{0}, b_{0}\right)-P \geq \hat{d}\left(a_{0},\left[a, b^{\prime}\right]\right)-P \\
& \geq \hat{d}\left(a_{0}, U^{+}(\bar{a}, i-6 \delta)\right)-P \underset{i \rightarrow \infty}{\rightarrow} \infty .
\end{aligned}
$$

The above inequality holds when $a, a^{\prime}, b, b^{\prime}$ are vertices, and by (35) it extends linearly to the case $\left(a, a^{\prime}, b, b^{\prime}\right) \in\left(V^{+}(\bar{a}, i) \times V^{+}\left(\bar{a}^{\prime}, i\right) \times V^{+}(\bar{b}, i) \times V^{+}(\bar{a}, i)\right) \cap S$. (38) and (39) also hold in this case, and they together imply that the limit in (37) equals $\infty$.

The same result is obtained when $\left(\bar{a}^{\prime}, \bar{b}\right)$ is a trivial side $\partial X$-pair. When $(\bar{a}, \bar{b})$ or $\left(\bar{a}^{\prime}, \bar{b}^{\prime}\right)$ is a trivial side $\partial X$-pair, the limit equals $-\infty$ by the same argument with inequalities reversed. This implies (f) and (g) and the existence of continuous extension to a function $\bar{X}^{\diamond} \rightarrow \overline{\mathbb{R}}$. Parts (a) though (e) now follow by continuity from the properties of the double difference in $X$.

It remains to show (h). Take $a, a^{\prime}, b, b^{\prime} \in X^{(0)}$, a geodesic $\left[b, b^{\prime}\right]$ in the 1skeleton and let $x$ and $x^{\prime}$ be some nearest point-projections of $a$ and $a^{\prime}$ to $\left[b, b^{\prime}\right]$, respectively. Orient $\left[b, b^{\prime}\right]$ from $b^{\prime}$ to $b$. It is an exercise in triangle inequality to see that $\left(a, a^{\prime} \mid b, b^{\prime}\right)$ is ${ }^{+}$equivalent to the signed distance $d\left(x, x^{\prime}\right)$, according to the orientation. Similarly, using Proposition 33. $\left\langle a, a^{\prime} \mid b, b^{\prime}\right\rangle$ is ${ }^{+}$equivalent to the signed distance $\hat{d}\left(x, x^{\prime}\right)$. But $d\left(x, x^{\prime}\right)$ and $\hat{d}\left(x, x^{\prime}\right)$ are ${ }^{x+}$ equivalent. This proves (h) for vertices in $X$. Now the ${ }^{\times+}$equivalence extends to all of $\bar{X}^{\diamond}$.

The double difference is continuous in $\bar{X}^{\diamond}$ and discontinuous at every quadruple in $\bar{X}^{4} \backslash \bar{X}^{\diamond}$. Theorem 35 immediately gives the continuous extension of the 
Gromov product $\langle a \mid b\rangle_{c}$ to the case when $(a, c, c, b) \in \bar{X}^{\diamond}$. This is equivalent to $(a, b, c) \in \bar{X}^{\triangleright}$, where

$$
\bar{X}^{\triangleright}:=\left\{(a, b, c) \in \bar{X}^{3} \mid c \in \partial X \rightarrow(a \neq c \text { and } b \neq c)\right\} .
$$

We have $X^{3} \subseteq \bar{X}^{\triangleright} \subseteq \bar{X}^{3}$ and Theorem 35 implies

Theorem 36 If $X$ is a hyperbolic complex, the Gromov product $\langle a \mid b\rangle_{c}$ with respect to $\hat{d}$ given by (33) extends to a continuous function $\langle\cdot \mid \cdot\rangle .: \bar{X}^{\triangleright} \rightarrow[0, \infty]$ such that $\langle a \mid b\rangle_{c}=\infty$ iff $c \in \partial X$ or $a=b \in \partial X$.

\subsection{More properties of double difference}

Lemma 37 Let $X$ be a hyperbolic complex. There exist $A \in[1, \infty)$ and $C \in$ $[0, \infty)$ depending only on $X$ such that for all $\left(a, a^{\prime}, b, b^{\prime}\right) \in\left(X^{(0)} \cup \partial X\right)^{4} \cap \bar{X}^{\diamond}$, $\alpha \in \operatorname{Geod}\left(a, a^{\prime}\right)$ and $\beta \in \operatorname{Geod}\left(b, b^{\prime}\right)$,

$$
\left.d(\alpha, \beta) \geq \max \left\{\left\langle b^{\prime}, a \mid a^{\prime}, b\right\rangle\right\},\left\langle b^{\prime}, a^{\prime} \mid a, b\right\rangle\right\} / A-C .
$$

Proof Take a distance-minimizing pair $\left(a_{0}, b_{0}\right), a_{0} \in \alpha, b_{0} \in \beta$, and set notations as in Figure 2. If $a=a^{\prime} \in \partial X$ or $b=b^{\prime} \in \partial X$ then the inequality obviously holds because both sides are $\infty$. Now we assume otherwise; this implies that $a_{0}, b_{0} \in X^{(0)}$. By the triangle inequality,

$\hat{d}\left(a_{0}, b_{0}\right) \geq \hat{d}(a, b)-\hat{d}\left(a, a_{0}\right)-\hat{d}\left(b, b_{0}\right)$ and $\hat{d}\left(a_{0}, b_{0}\right) \geq \hat{d}\left(a^{\prime}, b^{\prime}\right)-\hat{d}\left(a^{\prime}, a_{0}\right)-\hat{d}\left(b^{\prime}, b_{0}\right)$.

Since $a_{0} \in \alpha$ and $b_{0} \in \beta$, by Proposition 33

$$
\begin{aligned}
& \hat{d}\left(a_{0}, b_{0}\right) \\
& \geq \frac{1}{2}\left(\hat{d}(a, b)-\hat{d}\left(a, a_{0}\right)-\hat{d}\left(b, b_{0}\right)\right)+\frac{1}{2}\left(\hat{d}\left(a^{\prime}, b^{\prime}\right)-\hat{d}\left(a_{0}, a^{\prime}\right)-\hat{d}\left(b_{0}, b^{\prime}\right)\right) \\
& =\frac{1}{2}\left(\hat{d}(a, b)+\hat{d}\left(a^{\prime}, b^{\prime}\right)\right)-\frac{1}{2}\left(\hat{d}\left(a, a_{0}\right)+\hat{d}\left(a_{0}, a^{\prime}\right)\right)-\frac{1}{2}\left(\hat{d}\left(b, b_{0}\right)+\hat{d}\left(b_{0}, b^{\prime}\right)\right) \\
& \geq \frac{1}{2}\left(\hat{d}(a, b)+\hat{d}\left(a^{\prime}, b^{\prime}\right)\right)-\frac{1}{2}\left(\hat{d}\left(a, a^{\prime}\right)+C^{\prime}\right)+\frac{1}{2}\left(\hat{d}\left(b, b^{\prime}\right)+C^{\prime}\right) \\
& =\left\langle b^{\prime}, a \mid a^{\prime}, b\right\rangle-C^{\prime} .
\end{aligned}
$$

The same argument with $a$ and $a^{\prime}$ interchanged yields $\hat{d}\left(a_{0}, b_{0}\right) \geq\left\langle b^{\prime}, a^{\prime} \mid a, b\right\rangle-C^{\prime}$, so

$$
\hat{d}\left(a_{0}, b_{0}\right) \geq \max \left\{\left\langle b^{\prime}, a \mid a^{\prime}, b\right\rangle,\left\langle b^{\prime}, a^{\prime} \mid a, b\right\rangle\right\}-C^{\prime} .
$$

Since $\hat{d}$ and $d$ are quasiisometric (Theorem $32(\mathrm{a})$ ),

$$
\begin{aligned}
& d(\alpha, \beta)=d\left(a_{0}, b_{0}\right) \geq \hat{d}\left(a_{0}, b_{0}\right) / A-B \\
& \quad \geq \max \left\{\left\langle b^{\prime}, a \mid a^{\prime}, b\right\rangle,\left\langle b^{\prime}, a^{\prime} \mid a, b\right\rangle\right\} / A-C^{\prime} / A-B,
\end{aligned}
$$

so we denote $C:=C^{\prime} / A+B$. 
Proposition 38 Let $X$ be a hyperbolic complex. There exist constants $T \in$ $[0, \infty)$ and $\lambda \in[0,1)$ depending only on $X$ such that for all $(u, a, b, c) \in \bar{X}^{\diamond}$, if $\langle u, a \mid b, c\rangle \geq T$ or $\langle u, b \mid a, c\rangle \geq T$, then

$$
|\langle u, c \mid a, b\rangle| \leq \lambda^{\langle u, a \mid b, c\rangle} \leq 1 \quad \text { and } \quad|\langle u, c \mid a, b\rangle| \leq \lambda^{\langle u, b \mid a, c\rangle} \leq 1 \quad \text { (see Figure [5). }
$$

Equivalently,

if $\max \{\langle u, a \mid b, c\rangle,\langle u, b \mid a, c\rangle\} \geq T$ then $|\langle u, c \mid a, b\rangle| \leq \lambda^{\max \{\langle u, a \mid b, c\rangle,\langle u, b \mid a, c\rangle\}} \leq 1$.

Moreover, $\lambda$ can be taken arbitrarily close to 1 , with $T$ depending on $\lambda$.

Proof First assume that $(u, a, b, c) \in\left(X^{(0)} \cup \partial X\right)^{4} \cap \bar{X}^{\diamond}$. Take any $T \geq A(C+2 \delta)$, where $A$ and $C$ are from Lemma 37. Denote

$$
m:=\max \{\langle u, a \mid b, c\rangle,\langle u, b \mid a, c\rangle\} ;
$$

our assumption is that $m \geq T$. By Lemma 37 and our choice of $T$,

$$
d([a, b],[u, c]) \geq m / A-C \geq T / A-C \geq 2 \delta,
$$

then by Theorem 32 (d),

$$
|\langle u, c \mid a, b\rangle| \leq L \mu^{d([a, b],[u, c])} \leq L \mu^{m / A-C}=\left(L \mu^{-C}\right)\left(\mu^{1 / A}\right)^{m} .
$$

The right hand side decreases exponentially in $m$, so by taking $\lambda \in[0,1)$ sufficiently close to 1 and taking $T$ sufficiently large we can guarantee that the right hand side is at most $\lambda^{m}$ whenever $m \geq T$.

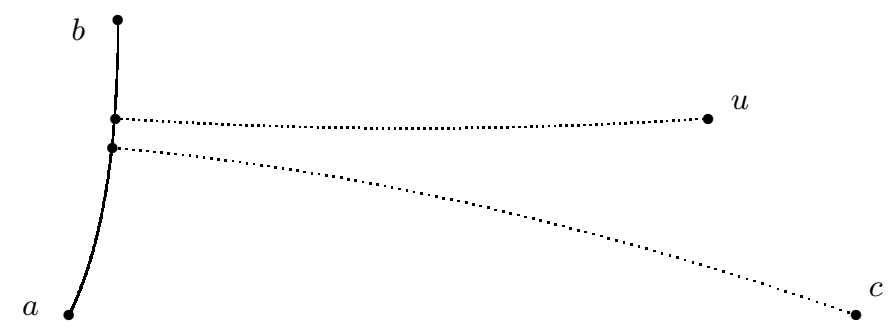

Figure 5: $\langle u, c \mid a, b\rangle$ is exponentially small

Now consider the general case $(u, a, b, c) \in \bar{X}^{\diamond}$, and let $m$ be as above. Let $D \in[0, \infty)$ be the maximal $\hat{d}$-diameter of a simplex in $X$, then $X^{(0)} \cup \partial X$ lies in the D-neighborhood of $\bar{X}$. If $u \in X$, then $u$ is in a simplex of $X$, and we replace $u$ with an arbitrary vertex $u^{\prime}$ of that simplex. If $u \in \partial X$, let $u^{\prime}:=u$. This replacement changes $\langle u, a \mid b, c\rangle$ and $\langle u, b \mid a, c\rangle$ by at most $D$. Doing the same for all four points, i.e. replacing $(u, a, b, c)$ with nearby 
$\left(u^{\prime}, a^{\prime}, b^{\prime}, c^{\prime}\right) \in\left(X^{(0)} \cup \partial X\right)^{4} \cap \bar{X}^{\diamond}$ changes $\langle u, a \mid b, c\rangle$ and $\langle u, b \mid a, c\rangle$ by at most $4 D$. So by the above argument,

$$
\begin{aligned}
& m \geq T+4 D \Rightarrow \max \left\{\left\langle u^{\prime}, a^{\prime} \mid b^{\prime}, c^{\prime}\right\rangle,\left\langle u^{\prime}, b^{\prime} \mid a^{\prime}, c^{\prime}\right\rangle\right\} \geq T \\
& \Rightarrow \quad\left|\left\langle u^{\prime}, c^{\prime} \mid a^{\prime}, b^{\prime}\right\rangle\right| \leq \lambda^{\max \left\{\left\langle u^{\prime}, a^{\prime} \mid b^{\prime}, c^{\prime}\right\rangle,\left\langle u^{\prime}, b^{\prime} \mid a^{\prime}, c^{\prime}\right\rangle\right\}} \leq \lambda^{m-4 D}=\lambda^{-4 D} \lambda^{m} .
\end{aligned}
$$

This holds for all nearby points $\left(u^{\prime}, a^{\prime}, b^{\prime}, c^{\prime}\right)$, so by the linearity formula (35), the same holds for $(u, a, b, c)$ :

$$
m \geq T+4 D \quad \Rightarrow \quad|\langle u, c \mid a, b\rangle| \leq \lambda^{-4 D} \lambda^{m} .
$$

Again, since the right hand side decreases exponentially in $m$, we can increase $\lambda \in[0,1)$ and $T \in[0, \infty)$ so that

$$
m \geq T \quad \Rightarrow \quad|\langle u, c \mid a, b\rangle| \leq \lambda^{m}
$$

\section{The cross ratio in $\bar{X}$}

Consider the double difference $\langle\cdot, \cdot \mid \cdot, \cdot\rangle$ given by Theorem 35

Definition 39 The cross-ratio in $\bar{X}$ is the function $\| \cdot, \cdot \cdot \cdot, \cdot]: \bar{X}^{\diamond} \rightarrow[0, \infty]$ defined by

$$
\left\|x, x^{\prime} \mid y, y^{\prime}\right\|:=e^{\left\langle x, x^{\prime} \mid y, y^{\prime}\right\rangle},
$$

with the convention $e^{-\infty}=0$ and $e^{\infty}=\infty$.

Formulas (32) and (40) can be applied to the standard metric on $\mathbb{H}^{3}$ in place of $\hat{d}$. In this case $\| \cdot, \cdot \cdot \cdot, \cdot] \|$ on the boundary $\partial \mathbb{H}^{3}=\mathbb{S}^{2}=\mathbb{C} \cup\{\infty\}$ is the absolute value of the usual cross ratio in $\mathbb{C} \cup\{\infty\}$, therefore the notation. The following is immediate from Theorem 35.

Theorem 40 The cross ratio $\| \cdot, \cdot \cdot \cdot, \cdot]$ in $\bar{X}$ defined above is continuous in $\bar{X}^{\diamond}$ and $\operatorname{Isom}(X)$-invariant.

This theorem generalizes the fact that Möbius transformations of $\mathbb{H}^{n}$, and, more generally, isometries of CAT $(-1)$-spaces, preserve the cross-ratio on the ideal boundary. In our case $\| \cdot, \cdot \cdot \cdot, \cdot]$ is defined on $\bar{X}^{\diamond}$, where $X$ is any hyperbolic complex. In particular, it is defined on all pairwise distinct quadruples of points in $\bar{X}$. Theorem 40 is also a sharp version of [35. Proposition 4.5] where quasiinvariance of a (non-continuous) cross-ratio (that is invariance up to an affine function under quasiisometries) was proved; and also of [23] where a measurable (non-continuous) invariant cross-ratio was constructed. 


\section{The symmetric join of $\bar{X}$}

Let $X$ be a hyperbolic complex. The functor $\otimes$ defined in section 2 can be applied to any topological space, in particular $\otimes \bar{X}$ makes sense, at least as a set. $\otimes \bar{X}$ is called the symmetric join of $\bar{X}$. In this section we extend the earlier constructions from $\otimes X$ to $\otimes \bar{X}$.

\subsection{Parametrizations of $\otimes \bar{X}$}

Recall that in 2.2 each line $\llbracket a, b \rrbracket$ of $x_{0}^{\otimes} X$ was parameterized by the interval $\left[-\left\langle b \mid x_{0}\right\rangle_{a},\left\langle a \mid x_{0}\right\rangle_{b}\right] \subseteq \mathbb{R}$, where $x_{0}$ is a fixed basepoint in $X$. Now suppose in addition that $X$ is a hyperbolic complex with the canonical metric $\hat{d}$ as in 6.1 and $a, b \in \bar{X}$. Then $\left(b, x_{0}, a\right),\left(a, x_{0}, b\right) \in \bar{X}^{\triangleright}$, hence by Theorem [36] $-\left\langle b \mid x_{0}\right\rangle_{a}$ and $\left\langle a \mid x_{0}\right\rangle_{b}$ are well-defined elements of $\overline{\mathbb{R}}$, except for the case $a=b \in \partial X$. In the case $a=b \in \partial X$ we let $-\left\langle b \mid x_{0}\right\rangle_{a}:=\left\langle a \mid x_{0}\right\rangle_{b}:=0$, so that $\llbracket a, a \rrbracket$ is identified with the trivial interval $[0,0] \subseteq \overline{\mathbb{R}}$. Note that the function $\langle\cdot \mid \cdot\rangle$. is not continuous at the triples $\left(b, x_{0}, a\right)$ with $a=b \in \partial X$.

This extends the parametrization in 2.2 to a parametrization ${ }_{x_{0}}^{\otimes} \bar{X}$ : lines $\llbracket a, b \rrbracket$ are identified with the closed intervals $\left[-\left\langle b \mid x_{0}\right\rangle_{a},\left\langle a \mid x_{0}\right\rangle_{b}\right]$ which are now subintervals of $\overline{\mathbb{R}}$ rather than of $\mathbb{R}$. The lines connecting distinct points at infinity are copies of $\overline{\mathbb{R}}$. The maps

$$
\llbracket a, b ; \cdot \rrbracket=\llbracket a, b ; \cdot \rrbracket_{x_{0}} \quad \text { and } \quad \llbracket a, b ; \cdot \rrbracket^{\prime}=\llbracket a, b ; \cdot \rrbracket_{x_{0}}^{\prime}
$$

are defined by the same formulas as in 2.2 .

The projection function $\llbracket \cdot, \cdot \mid \cdot \rrbracket$ is defined by the same formula as in Definition 7

$$
\left\langle a, a^{\prime} \mid b\right\rangle:=\left\langle a, a^{\prime} \mid b, x_{0}\right\rangle, \quad \llbracket a, a^{\prime} \mid b \rrbracket:=\llbracket a, a^{\prime} ;\left\langle a, a^{\prime} \mid b\right\rangle \rrbracket .
$$

But now, by continuity, the projection makes sense for any $a, a^{\prime}, b \in \bar{X}$.

Take any $x_{1} \in X$. By the same argument as in 2.10 $\llbracket a, b ; \cdot \rrbracket_{x_{1}}$ is the isometric orientation-preserving reparametrization of $\llbracket a, b \rrbracket$ whose origin $\llbracket a, b ; 0 \rrbracket_{x_{1}}$ is the projection of $x_{1}$ to $\llbracket a, b \rrbracket$.

\subsection{Actions on $\oplus X$}

The actions by $\mathbb{R}, \mathbb{Z}_{2}$, and $\operatorname{Isom}(X)$ on $\otimes X$ extend to $\otimes \bar{X}$ by the same formulas as in 2.6 2.7. 2.8. The formula (14) for the $\operatorname{Isom}(X)$-action indeed makes sense because by the triangle inequality $\left|\left\langle a, b \mid x_{0}, g^{-1} x_{0}\right\rangle\right| \leq \hat{d}\left(x_{0}, g^{-1} x_{0}\right)<\infty$ for all 
$a, b \in X$, and therefore by continuity for all $a, b \in \bar{X}$. For $a=b \in \bar{X}$ the formula implies

$$
g \llbracket a, a ; 0 \rrbracket=\llbracket g a, g a ; 0+\left\langle a, a \mid x_{0}, g^{-1} x_{0}\right\rangle \rrbracket=\llbracket g a, g a ; 0 \rrbracket,
$$

i.e. the $\operatorname{Isom}(X)$-action on $\otimes \bar{X}$ restricts to the usual $\operatorname{Isom}(X)$-action on $\bar{X}$.

These action satisfy Lemma 6 with $X$ replaced by $\bar{X}$, in particular, the $\mathbb{Z}_{2}$ and $\mathbb{R}$-actions fix $\bar{X}$ pointwise.

\subsection{The models $\otimes \bar{X}, \underset{x_{0}}{\circledast} \bar{X}, * \bar{X}, \underset{x_{0}}{*} \bar{X}, * \bar{X}$}

We use the same notations as in section 2 with $X$ replaced by $\bar{X}$. In accordance with 2.1 denote

$$
* \bar{X}:=(\otimes \bar{X}) \backslash \bar{X} .
$$

$* \bar{X}$ is called the open symmetric join of $\bar{X}$.

Just as in 2.3 and $2.4 \llbracket \cdot, \cdot ; \cdot \rrbracket^{\prime}$ induces a surjection $\llbracket \cdot, \cdot ; \cdot \rrbracket^{\prime}: \bar{X}^{2} \times \overline{\mathbb{R}} \rightarrow{ }_{x_{0}}^{\otimes} \bar{X}$ and bijections

$$
\begin{aligned}
& \llbracket \cdot, \cdot ; \cdot \rrbracket^{\prime}: \otimes \bar{X} \rightarrow \underset{x_{0}^{\circledast}}{\otimes}, \\
& \rrbracket \cdot, \cdot ; \cdot \rrbracket^{\prime}:\left(\bar{X}^{2} \backslash \bar{\Delta}\right) \times \mathbb{R} \rightarrow{ }_{x_{0}}^{*} \bar{X} \quad \text { and } \quad \rrbracket \cdot, \cdot ; \cdot \mathbb{\llbracket}^{\prime}: * \bar{X} \rightarrow *_{x_{0}}^{*} \bar{X}
\end{aligned}
$$

where $\bar{\Delta}$ is the diagonal of $\bar{X}^{2}$. The topology on $\underset{x_{0}}{\circledast} \bar{X}$ and $\underset{x_{0}^{*}}{*} \bar{X}$ is defined by either of these maps.

Denote

$$
* \bar{X}:=(\otimes \bar{X}) \backslash \partial X=* \bar{X} \cup X
$$

so we have $* \bar{X} \subseteq * \bar{X} \subseteq \otimes \bar{X}$.

\subsection{An extension of $\beta^{\times}$}

Pick $x, y \in * \bar{X}$, then they are of the form $x=\llbracket a, a^{\prime} ; s \rrbracket$ and $y=\llbracket b, b^{\prime} ; t \rrbracket$, $a, a^{\prime}, b, b^{\prime} \in \bar{X}$, for some appropriate $s$ and $t$; then necessarily $s, t \in \mathbb{R}$ and

$$
\left(a \in \partial X \rightarrow a^{\prime} \neq a\right) \text { and }\left(b \in \partial X \rightarrow b^{\prime} \neq b\right) .
$$

Theorem 35 and Theorem 36 imply that the formula

$$
\beta_{u}^{\times}(x, y):=\left\langle a \mid a^{\prime}\right\rangle_{u}+\left|s-\left\langle a, a^{\prime} \mid u\right\rangle\right|-\left\langle b \mid b^{\prime}\right\rangle_{u}-\left|t-\left\langle b, b^{\prime} \mid u\right\rangle\right|
$$

as in Definition 10 makes sense for all triples $(u, x, y) \in X \times(* \bar{X})^{2}$, and for such triples $\beta_{u}^{\times}(x, y) \in \mathbb{R}$. For each fixed $(x, y), \beta_{u}^{\times}(x, y)$ is Lipschitz in $u \in X$ : this follows from Theorem 11(a) by the continuity of the double difference, since $\otimes X$ is dense in $* \bar{X}$. We will see later in Theorem 55 that $\beta^{\times}$further extends to a continuous horofunction. 


\subsection{The map $\psi=\psi_{X}$}

Recall that $\left[a, a^{\prime}\right]$ is an arbitrary fixed choice of geodesic in $X^{(1)}$ connecting vertices $a$ and $a^{\prime}$. For each $a, a^{\prime} \in X$ make an arbitrary choice of projection point in $\operatorname{np}\left[a, a^{\prime} \mid x_{0}\right]$ denoted $\left[a, a^{\prime} \mid x_{0}\right]$. Proposition [33] says that the (images of the) usual geodesics in $\left(X^{(1)}, d\right)$ can be parameterized to become ${ }^{+}$geodesic in $(X, \hat{d})$, i.e. geodesic up to a uniform additive constant.

Define a map $\psi=\psi_{X}: \otimes \bar{X} \rightarrow \bar{X}$ as follows.

(a) If $x \in \bar{X}$, let $\psi(x):=x$.

(b) For $a, a^{\prime} \in \bar{X}$, consider the open interval parameterized as in 8.1, so it has the usual metric as a subinterval of $\overline{\mathbb{R}}$. Using Proposition 33 let $\psi$ map $\rrbracket a, a^{\prime} \llbracket{ }^{+}$isometrically to (]$a, a^{\prime}[, \hat{d})$, with a uniform constant.

(c) If both $a, a^{\prime} \in \partial X$, we additionally require the origins $\llbracket a, a^{\prime} ; 0 \rrbracket$ to map uniformly close to $\left[a, a^{\prime} \mid x_{0}\right]$.

The map $\psi{ }^{+}$commutes with the $\operatorname{Isom}(X)$-action on $\bar{X}$ and $\otimes \bar{X}$,

i.e. $\hat{d}(\psi(g x), g \psi(x))$ is uniformly bounded over all $g \in \operatorname{Isom}(X)$ and $x \in \otimes \bar{X}$. $\psi$ maps $* \bar{X}$ to $X$.

\subsection{Extending $d^{\times}$and $d_{*}$ to $\circledast \bar{X}$}

Define $d^{\times}$by the same formula

$$
d^{\times}(x, y):=\sup _{u \in X}\left|\beta_{u}^{\times}(x, y)\right|
$$

as in 3.2 but now applied to all $x, y \in \otimes \bar{X}$, i.e. $d^{\times}:(\otimes \bar{X})^{2} \rightarrow[0, \infty]$. Define

$$
\begin{aligned}
& \otimes \hat{d}(x, y):=\int_{-\infty}^{\infty} d^{\times}\left(r^{+} x, r^{+} y\right) \frac{e^{-|r|}}{2} d r \quad \text { and } \\
& \varphi(x):=\varphi_{X}(x):=\int_{-\infty}^{\infty} r^{+} x \frac{e^{-|r|}}{2} d r
\end{aligned}
$$

and denote for simplicity $d_{*}:=\otimes \hat{d}$. The formulas are the same as (16) and (18) in 3.3, but $d_{*}:(\oplus \bar{X})^{2} \rightarrow[0, \infty]$ and $\varphi: \oplus \bar{X} \rightarrow \oplus \bar{X}$. In what follows we deal with $\hat{d}, d^{\times}$and $d_{*}$ in the generalized sense, with infinite values allowed.

Proposition 41 For any hyperbolic complex $X$, the map $\psi$ in [8.5] viewed either as $\left(\otimes \bar{X}, d^{\times}\right) \rightarrow(\bar{X}, \hat{d})$ or as $\left(\otimes \bar{X}, d_{*}\right) \rightarrow(\bar{X}, \hat{d})$, is a ${ }^{+}$map. In particular, $d^{\times}$and $d_{*}$ take finite values on $* \bar{X}$. 
Proof We can discard the boundary since the values of $\hat{d}, d^{\times}$and $d_{*}$ are either 0 or $\infty$ and ${ }^{+}$equivalence is easily checked. $\psi$ is surjective, therefore the conclusion of the proposition is equivalent to saying that $\left(* \bar{X}, d^{\times}\right) \rightarrow(X, \hat{d})$ and $\left(* \bar{X}, d_{*}\right) \rightarrow(X, \hat{d})$ are ${ }^{+}$maps. By Lemma 18 it suffices to show that the first map $\psi:\left(* \bar{X}, d^{\times}\right) \rightarrow(X, \hat{d})$ is a ${ }^{+}$map.

It is not hard to see from the definitions of $\psi$ and $\ell$ (Definition 10, see also Figure 10, using hyperbolicity, Proposition 33 and Lemma 34, that

$$
\ell(u, x) \quad \text { and } \quad \hat{d}(u, \psi(x))
$$

are ${ }^{+}$equivalent as functions of $(u, x) \in X \times * \bar{X}$. Then by Lemma 1,

$$
\left|\beta_{u}^{\times}(x, y)\right|=|\ell(u, x)-\ell(u, y)| \quad \text { and } \quad|\hat{d}(u, \psi(x))-\hat{d}(u, \psi(y))|
$$

are ${ }^{+}$equivalent as functions of $(u, x, y) \in X \times(* \bar{X})^{2}$. Therefore

$$
d^{\times}(x, y)=\sup _{u \in X}\left|\beta_{u}^{\times}(x, y)\right| \quad \text { and } \quad \sup _{u \in X}|\hat{d}(u, \psi(x))-\hat{d}(u, \psi(y))|
$$

are ${ }^{+}$equivalent as functions of $(x, y) \in(* \bar{X})^{2}$. But since $\psi(x), \psi(y) \in X$ and by the triangle inequality, the last supremum is achieved at $u=\psi(x)$ and it equals $\hat{d}(\psi(x), \psi(y))$. So $d^{\times}(x, y)$ and $\hat{d}(\psi(x), \psi(y))$ are ${ }^{+}$equivalent, i.e. $\psi$ is $\mathrm{a}^{+}$map.

A subset $S$ of a (pseudo)metric space $Y$ is cobounded in $Y$ if there is $C \in[0, \infty)$ such that $Y$ is contained in the $C$-neighborhood of $S$.

Proposition 42 Let $X$ be a hyperbolic complex and $\psi$ be as in in 8.5, then $d^{\times}(y, \psi(y))$ and $d_{*}(y, \psi(y))$ are bounded uniformly over $y \in \otimes \bar{X}$. In particular, lines $\llbracket a, a^{\prime} \rrbracket$ in $\left(* \bar{X}, d^{\times}\right)$and in $\left(* \bar{X}, d_{*}\right)$ are uniformly close to geodesics $\left[a, a^{\prime}\right]$ in $X^{(1)} \cup \partial X$. Also, $X$ is cobounded both in $\left(* \bar{X}, d^{\times}\right)$and in $\left(* \bar{X}, d_{*}\right)$.

Proof Let $B$ be the constant of the ${ }^{+}$map $\psi:\left(* \bar{X}, d^{\times}\right) \rightarrow(X, \hat{d})$, and pick any $y \in \otimes \bar{X}$. By definition $\psi$ is idempotent, i.e. $\psi^{2}(y)=\psi(y) \in \bar{X}$, hence

$$
d^{\times}(y, \psi(y)) \leq \hat{d}\left(\psi(y), \psi^{2}(y)\right)+B=\hat{d}(\psi(y), \psi(y))+B=B .
$$

The same proof for $d_{*}$.

Proposition 43 For any hyperbolic complex $X$.

(a) $d_{*}, d^{\times}$and $\hat{d}$ coincide on $\bar{X}$, i.e. the canonical embeddings $(\bar{X}, \hat{d}) \hookrightarrow\left(\oplus \bar{X}, d^{\times}\right)$and $(\bar{X}, \hat{d}) \hookrightarrow\left(\oplus \bar{X}, d_{*}\right)$ are isometric.

(b) The map $\psi$ in 8.5 viewed either as $\left(\otimes \bar{X}, d^{\times}\right) \rightarrow(\bar{X}, \hat{d})$ or as $\left(\otimes \bar{X}, d_{*}\right) \rightarrow(\bar{X}, \hat{d})$ is a ${ }^{+}$isometry. 
Proof (a) follows from Theorem 16(c) (or [13(c)).

(b) follows from (a), propositions 41 and 42

Theorem 44 Let $X$ be a hyperbolic complex.

(a) The function $d^{\times}$above is a well-defined $\operatorname{Isom}(X)$-invariant pseudometric on $* \bar{X}$ independent of $x_{0}$. It is a generalized pseudometric on $\otimes \bar{X}$.

(b) The inclusion of each line $(\llbracket a, b \rrbracket,|\cdot|) \hookrightarrow\left(\otimes \bar{X}, d^{\times}\right), a, b \in \bar{X}$, is an isometric embedding.

Proof By Proposition 41] the values of $d^{\times}$on $(* \bar{X})^{2}$ are indeed in $[0, \infty)$. The rest follows as in the proof of Theorem 13

We summarize various properties that generalize from $X$ to $\bar{X}$.

Theorem 45 Let $X$ be a hyperbolic complex.

(a) The function $d_{*}$ in (46) is a well-defined $\operatorname{Isom}(X)$-invariant metric on $* \bar{X}$ independent of $x_{0}$. It is a generalized metric on $\oplus \bar{X}$.

(b) For each $r \in \mathbb{R}$, the map $r^{+}:\left(* \bar{X}, d_{*}\right) \rightarrow\left(* \bar{X}, d_{*}\right)$ is a bi-Lipschitz homeomorphism with constant $e^{|r|}$.

(c) The map $\varphi$ in 8.5 is a well-defined canonical surjection $\left(\oplus \bar{X}, d_{*}\right) \rightarrow\left(\oplus \bar{X}, d^{\times}\right)$whose restriction to each line $\left(\llbracket a, a^{\prime} \rrbracket, d_{*}\right)$ is an isometry onto $\left(\llbracket a, a^{\prime} \rrbracket, d^{\times}\right)$. In particular, each line $\llbracket a, a^{\prime} \rrbracket$ in $\left(\otimes X, d_{*}\right)$ can be parameterized to become a $d_{*}$-geodesic from $a$ to $a^{\prime}$.

(d) The restriction of $\varphi$ to $\bar{X}$ is the identity map $\left(\bar{X}, d_{*}\right) \rightarrow\left(\bar{X}, d^{\times}\right)$, and it is an isometry.

Proof By Proposition 41$]$ the values of $d_{*}$ on $(* \bar{X})^{2}$ are in $[0, \infty)$. The rest is shown as in theorems 14, 15, 16.

\section{The topology of $\otimes \bar{X}$}

\subsection{The topology $\mathcal{T}_{*}$ on $\otimes \bar{X}$}

We define a topology $\mathcal{T}_{*}$ on $\otimes \bar{X}$ as follows. Neighborhoods of a point $x \in * \bar{X}$ are $d_{*}$-balls centered at $x$. Neighborhoods of a point $x \in \partial X$ are the preimages of neighborhoods of $x$ in $\bar{X}$ under the ${ }^{+}$isometry $\psi:\left(\otimes \bar{X}, d_{*}\right) \rightarrow(\bar{X}, \hat{d})$ from 8.5 and Proposition 43. $\mathcal{T}_{*}$ induces the original topology on $\bar{X} \subseteq \otimes \bar{X}$.

Lemma 17(b) immediately extends to 
Lemma 46 For all $a, a^{\prime} \in \bar{X}$ with $a \neq a^{\prime}, \llbracket a, a^{\prime} ; \cdot \rrbracket^{\prime}: \overline{\mathbb{R}} \rightarrow\left(\llbracket a, a^{\prime} \rrbracket, \mathcal{T}_{*}\right)$ is a homeomorphism.

Lemma 47 (Convexity of $\mathcal{T}_{*}$ ) Let $X$ be a hyperbolic complex and $b \in \otimes \bar{X}$. For any neighborhood $N$ of $b$ in $\mathcal{T}_{*}$ there is a neighborhood $N^{\prime}$ of $b$ in $\mathcal{T}_{*}$ with the following properties.

(a) If $a, a^{\prime} \in \bar{X}$ and $x, x^{\prime} \in \llbracket a, a^{\prime} \rrbracket \cap N^{\prime}$, then the subinterval of $\llbracket a, a^{\prime} \rrbracket$ between $x$ and $x^{\prime}$ lies in $N$.

(b) If $a, a^{\prime} \in \bar{X}$ and $x, x^{\prime} \in \llbracket a, a^{\prime} \rrbracket \cap(\oplus \bar{X} \backslash N)$, then the subinterval of $\llbracket a, a^{\prime} \rrbracket$ between $x$ and $x^{\prime}$ lies in $\otimes \bar{X} \backslash N^{\prime}$.

Proof We will use the property that the lines, hence their subintervals, in $\otimes \bar{X}$ can be parameterized to become $d_{*}$-geodesic (Theorem 45(c)).

Assume first that $b \in * \bar{X}$. (a) follows from the fact that neighborhoods around $b$ can be taken to be $d_{*}$-balls. Suppose (b) does not hold, then there exist sequences $a_{i}$ and $a_{i}^{\prime}$ in $\bar{X}$, points $x_{i}, x_{i}^{\prime} \in \llbracket a_{i}, a_{i}^{\prime} \rrbracket \cap(\otimes \bar{X} \backslash N)$ and points $y_{i}$ between $x_{i}$ and $x_{i}^{\prime}$ such that $d_{*}\left(y_{i}, b\right) \rightarrow 0$. Then by Lemma 25] $d_{*}\left(a_{i}, b\right) \rightarrow 0$ or $d_{*}\left(a_{i}^{\prime}, b\right) \rightarrow 0$, for example the former. Since $x_{i}$ lie between $a_{i}$ and $y_{i}$, both converging to $b$ in the metric $d_{*}$, then $x_{i}$ must also converge to $b$, which is a contradiction with the choice of $x_{i}$.

Now assume $b \in \partial X$. By Proposition 42 lines $\llbracket a, a^{\prime} \rrbracket$ in $\left(* \bar{X}, d_{*}\right)$ are uniformly close to geodesics $\left[a, a^{\prime}\right]$ in the 1-skeleton. Then both (a) and (b) follow from lemmas 30 and 31 .

\subsection{The topology of $* \bar{X}$}

Our goal is to prove the following.

Proposition 48 Let $X$ be a hyperbolic complex with the standard metric $\hat{d}$. The metric $d_{*}$ from 8.6 induces the original topology on the open symmetric join $x_{0}^{*} \bar{X}$ described in 8.3. Equivalently, the map from 8.3 viewed as

$$
\rrbracket \cdot, \cdot ; \cdot \llbracket^{\prime}:\left(\bar{X}^{2} \backslash \bar{\Delta}\right) \times \mathbb{R} \rightarrow\left(x_{0}^{*} \bar{X}, d_{*}\right)
$$

is a homeomorphism, where $\bar{\Delta}$ is the diagonal of $\bar{X}^{2}$.

Lemma 49 Suppose $b, a_{i}, u_{i} \in \otimes \bar{X}, a_{i} \rightarrow b$, and $\left\langle a_{i}, b \mid u_{i}\right\rangle \nrightarrow 0$. Then $b \in \partial X$ and it is possible to take a subsequence so that $u_{i} \rightarrow b$. 
Proof If $b \in X$, then $\left\langle a_{i}, b \mid u_{i}\right\rangle=\left\langle a_{i}, b \mid u_{i}, x_{0}\right\rangle \leq \hat{d}\left(a_{i}, b\right) \rightarrow 0$ which contradict our assumptions. Therefore $b$ must be in $\partial X$.

Now suppose to the contrary that $u_{i}$ stays away from a neighborhood $V^{+}$of $b$ for all $i$. Using the definition of neighborhoods one checks that $\left(a_{i}, x_{0} \mid u_{i}, b\right) \rightarrow$ $\infty$, hence by Theorem 35(h), $\left\langle a_{i}, x_{0} \mid u_{i}, b\right\rangle \rightarrow \infty$, so by Theorem 38 $\left|\left\langle a_{i}, b \mid u_{i}\right\rangle\right|$ $=\left|\left\langle a_{i}, b \mid u_{i}, x_{0}\right\rangle\right| \leq \lambda^{\left\langle a_{i}, x_{0} \mid u_{i}, b\right\rangle} \rightarrow 0$, which is a contradiction.

Lemma 50 Let $X$ be a hyperbolic complex, $I \subseteq \mathbb{R}$ be a compact interval, and $a, a^{\prime}, b, b^{\prime} \in \bar{X}$. If $b, b^{\prime} \in \partial X$, assume $b \neq b^{\prime}$. Then

$$
d^{\times}\left(\llbracket a, a^{\prime} ; t \rrbracket, \llbracket b, b^{\prime} ; t \rrbracket\right) \rightarrow 0 \quad \text { as } a \rightarrow b \text { and } a^{\prime} \rightarrow b^{\prime} \text { in } \bar{X}
$$

uniformly on $t \in I$.

Proof Suppose not, then there is $\epsilon>0$ and there are sequences $a_{i} \rightarrow b$, $a_{i}^{\prime} \rightarrow b^{\prime}$ in $\bar{X}$ and $t_{i} \in I$ such that

$$
d^{\times}\left(\llbracket a_{i}, a_{i}^{\prime} ; t_{i} \rrbracket, \llbracket b, b^{\prime} ; t_{i} \rrbracket\right) \geq \epsilon \text { for all } i .
$$

Then by the definition of $d^{\times}$there is a sequence $u_{i} \in X$ such that

$$
\beta_{u_{i}}^{\times}\left(\llbracket a_{i}, a_{i}^{\prime} ; t_{i} \rrbracket, \llbracket b, b^{\prime} ; t_{i} \rrbracket\right) \geq \frac{\epsilon}{2} \text { for all } i .
$$

Taking subsequences we can assume that one of the following four cases holds.

Case $1 \quad t_{i} \leq\left\langle a_{i}, a_{i}^{\prime} \mid u_{i}\right\rangle$ and $t_{i} \leq\left\langle b, b^{\prime} \mid u_{i}\right\rangle$ for all $i$

By the definition of $\beta^{\times}$(45),

$$
\begin{aligned}
& \beta_{u_{i}}^{\times}\left(\llbracket a_{i}, a_{i}^{\prime} ; t_{i} \rrbracket, \llbracket b, b^{\prime} ; t_{i} \rrbracket\right)=\left\langle a_{i} \mid a_{i}^{\prime}\right\rangle_{u_{i}}-t_{i}+\left\langle a_{i}, a_{i}^{\prime} \mid u_{i}\right\rangle-\left\langle b \mid b^{\prime}\right\rangle_{u_{i}}+t_{i}-\left\langle b, b^{\prime} \mid u_{i}\right\rangle \\
& =\left\langle a_{i} \mid a_{i}^{\prime}\right\rangle_{u_{i}}+\left\langle a_{i}, a_{i}^{\prime} \mid u_{i}\right\rangle-\left\langle b \mid b^{\prime}\right\rangle_{u_{i}}-\left\langle b, b^{\prime} \mid u_{i}\right\rangle .
\end{aligned}
$$

Denote the last expression $\beta_{i}$. By computation,

$$
\beta_{i}=2\left\langle a_{i}, b \mid u_{i}\right\rangle-\left\langle a_{i}, b \mid a_{i}^{\prime}\right\rangle-\left\langle a_{i}^{\prime}, b^{\prime} \mid b\right\rangle .
$$

The last two terms approach 0 as $i \rightarrow \infty$.

We claim that $\beta_{i} \rightarrow 0$. If not then $\left\langle a_{i}, b \mid u_{i}\right\rangle \not 0$, and by Lemma 49, $b \in \partial X$ and, after taking a subsequence, $u_{i} \rightarrow b$. Then by Theorem 35(g), $\left\langle a_{i}, a_{i}^{\prime} \mid u_{i}\right\rangle \rightarrow$ $-\infty$, so by the assumptions of Case $1, t_{i} \rightarrow-\infty$. This is impossible since $t_{i} \in I$. This proves $\beta_{i} \rightarrow 0$.

The condition $\beta_{i} \rightarrow 0$ contradicts (47).

Case $2 \quad\left\langle a_{i}, a_{i}^{\prime} \mid u_{i}\right\rangle \leq t_{i}$ and $\left\langle b, b^{\prime} \mid u_{i}\right\rangle \leq t_{i}$ for all $i$ 
This is the same as Case 1 by interchanging $a_{i} \leftrightarrow a_{i}^{\prime}, b \leftrightarrow b^{\prime}, t_{i} \leftrightarrow-t_{i}$.

Case $3\left\langle b, b^{\prime} \mid u_{i}\right\rangle \leq t_{i} \leq\left\langle a_{i}, a_{i}^{\prime} \mid u_{i}\right\rangle$ for all $i$

By the definition of $\beta^{\times}$we have

$$
\begin{aligned}
& \beta_{u_{i}}^{\times}\left(\llbracket a_{i}, a_{i}^{\prime} ; t_{i} \rrbracket, \llbracket b, b^{\prime} ; t_{i} \rrbracket\right) \\
& \quad=\left\langle a_{i} \mid a_{i}^{\prime}\right\rangle_{u_{i}}-t_{i}+\left\langle a_{i}, a_{i}^{\prime} \mid u_{i}\right\rangle-\left\langle b \mid b^{\prime}\right\rangle_{u_{i}}-t_{i}+\left\langle b, b^{\prime} \mid u_{i}\right\rangle .
\end{aligned}
$$

First we let $t_{i}:=\left\langle b, b^{\prime} \mid u_{i}\right\rangle$, then

$$
\beta_{u_{i}}^{\times}\left(\llbracket a_{i}, a_{i}^{\prime} ; t_{i} \rrbracket, \llbracket b, b^{\prime} ; t_{i} \rrbracket\right)=\left\langle a_{i} \mid a_{i}^{\prime}\right\rangle_{u_{i}}-\left\langle b, b^{\prime} \mid u_{i}\right\rangle+\left\langle a_{i}, a_{i}^{\prime} \mid u_{i}\right\rangle-\left\langle b \mid b^{\prime}\right\rangle_{u_{i}} .
$$

This expression is the same as in (48), so we call it $\beta_{i}$. The same argument as in Case 1 shows that $\beta_{i} \rightarrow 0$.

Now let $t_{i}:=\left\langle a_{i}, a_{i}^{\prime} \mid u_{i}\right\rangle$, then

$$
\beta_{u_{i}}^{\times}\left(\llbracket a_{i}, a_{i}^{\prime} ; t_{i} \rrbracket, \llbracket b, b^{\prime} ; t_{i} \rrbracket\right)=\left\langle a_{i} \mid a_{i}^{\prime}\right\rangle_{u_{i}}-\left\langle b \mid b^{\prime}\right\rangle_{u_{i}}-\left\langle a_{i}, a_{i}^{\prime} \mid u_{i}\right\rangle+\left\langle b, b^{\prime} \mid u_{i}\right\rangle .
$$

We call the last expression $\beta_{i}^{\prime}$. It is obtained from $\beta_{i}$ by interchanging $a_{i} \leftrightarrow a_{i}^{\prime}$, $b \leftrightarrow b^{\prime}$, and a similar argument shows that $\beta_{i}^{\prime} \rightarrow 0$.

By the assumptions of Case 3, (49) lies between $\beta_{i}$ and $\beta_{i}^{\prime}$, hence it converges to 0 . This contradicts (47).

Case $4\left\langle a_{i}, a_{i}^{\prime} \mid u_{i}\right\rangle \leq t_{i} \leq\left\langle b, b^{\prime} \mid u_{i}\right\rangle$ for all $i$

This is the same as Case 3 by interchanging $a_{i} \leftrightarrow a_{i}^{\prime}, b \leftrightarrow b^{\prime}, t_{i} \leftrightarrow-t_{i}$.

Lemma 51 Under the assumptions of Lemma 50 ,

$$
d^{\times}\left(\llbracket a, a^{\prime} ; t \rrbracket^{\prime}, \llbracket b, b^{\prime} ; t \rrbracket^{\prime}\right) \rightarrow 0 \quad \text { as } a \rightarrow b \text { and } a^{\prime} \rightarrow b^{\prime} \text { in } \bar{X}
$$

uniformly on $t \in I$.

Proof Recall from 8.1 that $\llbracket a, a^{\prime} \rrbracket$ is a copy of the interval $\left[\alpha, \alpha^{\prime}\right] \subseteq \overline{\mathbb{R}}$, where $\alpha:=-\left\langle a^{\prime} \mid x_{0}\right\rangle_{a}$ and $\alpha^{\prime}:=\left\langle a \mid x_{0}\right\rangle_{a^{\prime}}$. Similarly, $\llbracket b, b^{\prime} \rrbracket$ is a copy of $\left[\beta, \beta^{\prime}\right] \subseteq \mathbb{R}$ where $\beta:=-\left\langle b^{\prime} \mid x_{0}\right\rangle_{b}$ and $\beta^{\prime}:=\left\langle b \mid x_{0}\right\rangle_{b^{\prime}}$. If $a \rightarrow b$ and $a^{\prime} \rightarrow b^{\prime}$ in $\bar{X}$, then $\alpha \rightarrow \beta$ and $\alpha^{\prime} \rightarrow \beta^{\prime}$ in $\overline{\mathbb{R}}$. (When some of $a, a^{\prime}, b, b^{\prime}$ are in $\partial X$, this is an exercise for the Gromov product defined by the word metric $d$, and then use Theorem [35(h) to show the same for $\langle\cdot \mid \cdot\rangle$. .)

Using Lemma 3 we denote

$$
\begin{aligned}
& A:=\theta^{\prime}\left[\alpha, \alpha^{\prime} ; t\right]=\theta\left[\alpha, \alpha^{\prime} ; t\right]+\left(e^{-|t-\alpha|}-e^{-\left|t-\alpha^{\prime}\right|}\right) / 2 \text { and } \\
& B:=\theta^{\prime}\left[\beta, \beta^{\prime} ; t\right]=\theta\left[\beta, \beta^{\prime} ; t\right]+\left(e^{-|t-\beta|}-e^{-\left|t-\beta^{\prime}\right|}\right) / 2,
\end{aligned}
$$


then

$|B-A| \leq\left|\theta\left[\beta, \beta^{\prime} ; t\right]-\theta\left[\alpha, \alpha^{\prime} ; t\right]\right|+\left|e^{-|t-\beta|}-e^{-|t-\alpha|}\right| / 2+\left|e^{-\left|t-\alpha^{\prime}\right|}-e^{-\left|t-\beta^{\prime}\right|}\right| / 2$.

This implies that for each compact $J \subseteq \mathbb{R}$,

$$
|B-A| \rightarrow 0 \quad \text { as } a \rightarrow b \text { and } a^{\prime} \rightarrow b^{\prime} \text { in } \bar{X}
$$

uniformly on $t \in J$. By (17) and since the map $\llbracket a, a^{\prime} ; \rrbracket^{\prime}: \mathbb{R} \rightarrow\left(\llbracket a, a^{\prime} \rrbracket, d^{\times}\right)$is non-expanding,

$$
\begin{aligned}
& d^{\times}\left(\llbracket a, a^{\prime} ; t \rrbracket^{\prime}, \llbracket b, b^{\prime} ; t \rrbracket^{\prime}\right)=d^{\times}\left(\llbracket a, a^{\prime} ; \theta^{\prime}\left[\alpha, \alpha^{\prime} ; t\right] \rrbracket, \llbracket b, b^{\prime} ; \theta^{\prime}\left[\beta, \beta^{\prime} ; t\right] \rrbracket\right) \\
& =d^{\times}\left(\llbracket a, a^{\prime} ; A \rrbracket, \llbracket b, b^{\prime} ; B \rrbracket\right) \leq d^{\times}\left(\llbracket a, a^{\prime} ; A \rrbracket, \llbracket a, a^{\prime} ; B \rrbracket\right)+d^{\times}\left(\llbracket a, a^{\prime} ; B \rrbracket, \llbracket b, b^{\prime} ; B \rrbracket\right) \\
& \leq|B-A|+d^{\times}\left(\llbracket a, a^{\prime} ; B \rrbracket, \llbracket b, b^{\prime} ; B \rrbracket\right) .
\end{aligned}
$$

This, Lemma 50 and (50) imply Lemma 51.

Proposition 52 Let $X$ be a hyperbolic complex, $s, t \in \overline{\mathbb{R}}, a, a^{\prime}, b, b^{\prime} \in \bar{X}$. Then

$$
\llbracket a, a^{\prime} ; s \rrbracket^{\prime} \rightarrow \llbracket b, b^{\prime} ; t \rrbracket^{\prime} \quad \text { in }\left(\otimes \bar{X}, \mathcal{T}_{*}\right) \quad \text { as } \quad a \rightarrow b, a^{\prime} \rightarrow b^{\prime} \text { in } \bar{X} \text { and } s \rightarrow t \text { in } \overline{\mathbb{R}} \text {. }
$$

Equivalently, the map $\llbracket \cdot, \cdot ; \cdot \rrbracket^{\prime}: \bar{X}^{2} \times \overline{\mathbb{R}} \rightarrow\left(\otimes \bar{X}, \mathcal{T}_{*}\right)$ is continuous.

Proof First assume $b=b^{\prime}$. Since $a \rightarrow b$ and $a^{\prime} \rightarrow b^{\prime}=b$, then by the convexity of $\mathcal{T}_{*}$ (Lemma 47), all the points of $\llbracket a, a^{\prime} \rrbracket$ must converge to $b$ as well. So now we assume $b \neq b^{\prime}$.

Assume $t \in \mathbb{R}$. For any $s \in \mathbb{R}$, since $\llbracket a, a^{\prime} ; \rrbracket^{\prime}: \mathbb{R} \rightarrow\left(\llbracket a, a^{\prime} \rrbracket, d^{\times}\right)$is nonexpanding,

$$
\begin{aligned}
& d^{\times}\left(\llbracket a, a^{\prime} ; r+s \rrbracket^{\prime}, \llbracket b, b^{\prime} ; r+t \rrbracket^{\prime}\right) \\
& \leq d^{\times}\left(\llbracket a, a^{\prime} ; r+s \rrbracket^{\prime}, \llbracket a, a^{\prime} ; r+t \rrbracket^{\prime}\right)+d^{\times}\left(\llbracket a, a^{\prime} ; r+t \rrbracket^{\prime}, \llbracket b, b^{\prime} ; r+t \rrbracket^{\prime}\right) \\
& \leq|t-s|+d^{\times}\left(\llbracket a, a^{\prime} ; r+t \rrbracket^{\prime}, \llbracket b, b^{\prime} ; r+t \rrbracket^{\prime}\right) .
\end{aligned}
$$

Pick any $\epsilon>0$. By Lemma 51 there exist neighborhoods $N_{b}$ of $b$ and $N_{b^{\prime}}$ of $b^{\prime}$ in $\bar{X}$ such that

$$
d^{\times}\left(\llbracket a, a^{\prime} ; r+t \rrbracket^{\prime}, \llbracket b, b^{\prime} ; r+t \rrbracket^{\prime}\right) \leq \epsilon
$$

for all $a \in N_{b}, a^{\prime} \in N_{b^{\prime}}$ and $r \in\left[-\frac{1}{\epsilon}, \frac{1}{\epsilon}\right]$. Also for $r \in\left(-\infty,-\frac{1}{\epsilon}\right] \cup\left[\frac{1}{\epsilon}, \infty\right)$, we have

$$
\begin{aligned}
& d^{\times}\left(\llbracket a, a^{\prime} ; r+t \rrbracket^{\prime}, \llbracket b, b^{\prime} ; r+t \rrbracket^{\prime}\right) \leq d^{\times}\left(\llbracket a, a^{\prime} ; r+t \rrbracket^{\prime}, \llbracket a, a^{\prime} ; t \rrbracket^{\prime}\right)+ \\
& d^{\times}\left(\llbracket a, a^{\prime} ; t \rrbracket^{\prime}, \llbracket b, b^{\prime} ; t \rrbracket^{\prime}\right)+d^{\times}\left(\llbracket b, b^{\prime} ; t \rrbracket^{\prime}, \llbracket b, b^{\prime} ; r+t \rrbracket^{\prime}\right) \leq 2|r|+\epsilon .
\end{aligned}
$$


Then by the definition of $d_{*}$,

$$
\begin{aligned}
& d_{*}\left(\llbracket a, a^{\prime} ; s \rrbracket^{\prime}, \llbracket b, b^{\prime} ; t \rrbracket^{\prime}\right)=\int_{-\infty}^{\infty} d^{\times}\left(\llbracket a, a^{\prime} ; r+s \rrbracket^{\prime}, \llbracket b, b^{\prime} ; r+t \rrbracket^{\prime}\right) \frac{e^{-|r|}}{2} d r \\
& \leq \int_{-\frac{1}{\epsilon}}^{\frac{1}{\epsilon}}(|t-s|+\epsilon) \frac{e^{-|r|}}{2} d r+\int_{\frac{1}{\epsilon}}^{\infty}(2|r|+\epsilon) \frac{e^{-|r|}}{2} d r+\int_{-\infty}^{-\frac{1}{\epsilon}}(2|r|+\epsilon) \frac{e^{-|r|}}{2} d r \\
& \leq|t-s|+\epsilon+2(1 / \epsilon+1) e^{-1 / \epsilon} \rightarrow 0 \quad \text { as } s \rightarrow t \text { and } \epsilon \searrow 0 .
\end{aligned}
$$

This implies the statement of the proposition.

Assume $t=\infty$, then $\llbracket b, b^{\prime} ; t \rrbracket^{\prime}=\llbracket b, b^{\prime} ; \infty \rrbracket^{\prime}=b^{\prime} \in \bar{X}$. By Lemma 46, $\llbracket b, b^{\prime} ; \cdot \rrbracket^{\prime}$ : $\overline{\mathbb{R}} \rightarrow\left(\llbracket b, b^{\prime} \rrbracket, \mathcal{T}_{*}\right)$ is a homeomorphism, therefore by taking $s \in \mathbb{R}$ close to $\infty$ we can make the whole interval $\llbracket b, b^{\prime} ;[s, \infty] \rrbracket^{\prime}$ arbitrarily close to $b^{\prime}$. For a fixed $s \in \mathbb{R}$, by the above argument, by taking $\left(a, a^{\prime}\right)$ close to $\left(b, b^{\prime}\right)$ in $T_{*}$ we can make $d_{*}\left(\llbracket a, a^{\prime} ; s \rrbracket^{\prime}, \llbracket b, b^{\prime} ; s \rrbracket^{\prime}\right)$ arbitrarily small. This implies that both $\llbracket a, a^{\prime} ; s \rrbracket^{\prime}$ and $a^{\prime}=\llbracket a, a^{\prime} ; \infty \rrbracket^{\prime}$ are arbitrarily close to $b^{\prime}$, then by the convexity of $T_{*}$, the interval $\llbracket a, a^{\prime} ;[s, \infty] \rrbracket^{\prime}$ can be made arbitrarily close to $b^{\prime}$. The case $t=-\infty$ is similar.

Lemma 53 Let $X$ be a hyperbolic complex. Let $a \in \bar{X}$ and $x_{i}=\llbracket a_{i}, a_{i}^{\prime} ; s_{i} \rrbracket^{\prime} \in \otimes \bar{X}$ be a sequence such that $x_{i} \rightarrow a$ in $\left(\otimes \bar{X}, \mathcal{T}_{*}\right)$. Then $a_{i} \rightarrow a$ or $a_{i}^{\prime} \rightarrow a$ in $\bar{X}$.

Proof First consider the case $a \in X$, so our assumption is that $d_{*}\left(x_{i}, a\right) \rightarrow 0$. The proof is word-by-word as in Lemma 25.

Now we assume $a \in \partial X$. If, to the contrary, some subsequences $a_{j}$ and $a_{j}^{\prime}$ stay away from a neighborhood $V$ of $a$ in $\bar{X}$, then by the convexity of $\mathcal{T}_{*}$ all the lines $\llbracket a_{j}, a_{j}^{\prime} \rrbracket$, and hence all $x_{j}$, must stay away from some smaller neighborhood of $a$, which contradicts $x_{j} \rightarrow a$.

Proof of Proposition 48 We will show that the identity map $x_{0}^{*} \bar{X} \rightarrow\left(x_{0}^{*} \bar{X}, d_{*}\right)$ and its inverse are continuous at every point $y=\rrbracket b, b^{\prime} ; t \rrbracket^{\prime}$ in $x_{0}^{*} \bar{X}$.

Proposition 52 says that $x_{0}^{*} \bar{X} \rightarrow\left(x_{0}^{*} \bar{X}, d_{*}\right)$ is continuous at $y$. Suppose that the inverse map $\left({ }_{x_{0}}^{*} \bar{X}, d_{*}\right) \rightarrow x_{0}^{*} \bar{X}$ is not continuous at $y$. We obtain a contradiction just as in the proof of Proposition [20, but using $\bar{X}$, Proposition [52] and Lemma 53 . 


\subsection{Properness of $* \bar{X}$}

A metric space $Y$ is called proper if each closed ball in $Y$ is compact. Recall that $* \bar{X}=\oplus \bar{X} \backslash \partial X$.

Proposition 54 For any hyperbolic complex $X,\left(* \bar{X}, d_{*}\right)$ is proper.

Proof Let $B_{d_{*}}(r)$ be the closed ball in $\left(* \bar{X}, d_{*}\right)$ of radius $r$ centered at the basepoint $x_{0} \in X$. Since $X$ is cobounded in $\left(* \bar{X}, d_{*}\right)$, any ball lies in $B_{d_{*}}(r)$ for sufficiently large $r$. Since $\left(* \bar{X}, d_{*}\right)$ is a metric space, it suffices to show the sequential compactness of $B_{d_{*}}(r)$.

Fix $r \geq 0$ and pick $x=\llbracket a, a^{\prime} ; s \rrbracket^{\prime} \in B_{d_{*}}(r)$. We have by the definitions of $\ell$ and $d^{\times}$and Lemma 18 .

$$
\begin{aligned}
& \left\langle a \mid a^{\prime}\right\rangle_{x_{0}} \leq\left\langle a \mid a^{\prime}\right\rangle_{x_{0}}+\left|s-\left\langle a, a^{\prime} \mid x_{0}\right\rangle\right|=\ell\left(x_{0}, x\right)=\left|\ell\left(x_{0}, x_{0}\right)-\ell\left(x_{0}, x\right)\right| \\
& \leq \sup _{u \in X}\left|\ell\left(u, x_{0}\right)-\ell(u, x)\right|=d^{\times}\left(x_{0}, x\right) \leq d_{*}\left(x_{0}, x\right)+2 \leq r+2 .
\end{aligned}
$$

Similarly $|s|=\left|s-\left\langle a, a^{\prime} \mid x_{0}\right\rangle\right| \leq r+2$. Let $x_{i}=\llbracket a_{i}, a_{i}^{\prime} ; s_{i} \rrbracket$ be a sequence in $B_{d_{*}}(r)$. We have

$$
\left\langle a_{i} \mid a_{i}^{\prime}\right\rangle_{x_{0}} \leq r+2 \text { and }\left|s_{i}\right| \leq r+2 \text { for all } i .
$$

After replacing $\left\{x_{i}\right\}$ with a subsequence, $s_{i}$ converges to some $\bar{s}$ with $|\bar{s}| \leq r+2$. By Theorem 36] the function $\langle\cdot \mid \cdot\rangle_{x_{0}}: \bar{X}^{2} \rightarrow[0, \infty]$ is continuous, hence the set

$$
\left\{\left(a, a^{\prime}\right) \in \bar{X}^{2} \mid\left\langle a \mid a^{\prime}\right\rangle_{x_{0}} \leq r+2\right\}
$$

is closed in $\bar{X}^{2}$ and therefore compact, so after replacing $\left\{x_{i}\right\}$ with a subsequence again, $a_{i}$ and $a_{i}^{\prime}$ converge to some $\bar{a}$ and $\bar{a}^{\prime}$ in $\bar{X}$, respectively, satisfying $\left\langle\bar{a} \mid \bar{a}^{\prime}\right\rangle_{x_{0}} \leq r+2$.

Denote $\bar{x}:=\llbracket \bar{a}, \bar{a}^{\prime} ; \bar{s} \rrbracket$. By Proposition $52, d_{*}\left(x_{i}, \bar{x}\right) \rightarrow 0$. Also

$$
d_{*}\left(x_{0}, \bar{x}\right) \leq d_{*}\left(x_{0}, x_{i}\right)+d_{*}\left(x_{i}, \bar{x}\right) \leq r+d_{*}\left(x_{i}, \bar{x}\right) \underset{i \rightarrow \infty}{\rightarrow} r
$$

so $\bar{x} \in B_{d_{*}}(r)$.

\section{Horofunctions and horospheres in $\bar{X}$ and $\otimes \bar{X}$}

In the classical case of $\mathbb{H}^{n}$, a horofunction, or Busemann function, $\beta_{u}$ is defined with respect to a point at infinity, $u \in \partial \mathbb{H}^{n}$. A horofunction in a hyperbolic 
metric space related to a point $u$ on the ideal boundary was usually defined either depending on a geodesic ray converging to $u$ ([4, III.3.4], [27, 7.5.C]), or satisfied the natural identities only "up to a constant" ([25, Chapitre 8]), or was a measurable function (23]).

In this section we show that the metric $\hat{d}$ allows defining a continuous horofunction $\beta_{u}^{\times}$on any hyperbolic complex $X$; the horofunction will satisfy sharp identities as in the classical $\mathbb{H}^{n}$ case. We will also allow $u \in X$; in this case $\beta_{u}^{\times}(x, y)$ will be the distance cocycle: $\beta_{u}^{\times}(x, y)=\hat{d}(u, x)-\hat{d}(u, y)$. Moreover, $\beta_{u}^{\times}(x, y)$ will be defined not only for $x, y \in X$ but also for $x, y \in * X$, and more.

Let $X$ be a hyperbolic complex. Recall that $* \bar{X}=(\otimes \bar{X}) \backslash \partial X=* \bar{X} \cup X$.

Theorem 55 Let $X$ be a hyperbolic complex and $\beta^{\times}$be the cocycle from Definition [10,

(a) Put the pseudometric $\hat{d}+d^{\times}+d^{\times}$on $X \times(* \bar{X})^{2}$. Then $\beta^{\times}$extends to a Lipschitz function $\beta^{\times}: X \times(* \bar{X})^{2} \rightarrow \mathbb{R}$ independent of $x_{0}$.

$\left(\mathrm{a}^{\prime}\right)$ Put the usual topology on $\bar{X}$ and the topology induced by $d^{\times}$on $* \bar{X}$. Then $\beta^{\times}$further extends to a continuous function $\beta^{\times}: \bar{X} \times(* \bar{X})^{2} \rightarrow \mathbb{R}$ independent of $x_{0}$.

(b) $\beta_{u}^{\times}$is $\mathbb{Z}_{2}$-invariant in each variable: $\beta_{u}^{\times}(x, y)=\beta_{u}^{\times}\left(x^{\star}, y\right)=\beta_{u}^{\times}\left(x, y^{\star}\right)$.

(c) $\beta_{u}^{\times}$satisfies the cocycle condition $\beta_{u}^{\times}(x, y)+\beta_{u}^{\times}(y, z)=\beta_{u}^{\times}(x, z)$.

(d) $\beta^{\times}$is $\operatorname{Isom}(X)$-invariant: $\beta_{g u}^{\times}(g x, g y)=\beta_{u}^{\times}(x, y)$ for $g \in \operatorname{Isom}(X)$.

(e) $\beta^{\times}$is isometric on lines: for all $a, b \in \bar{X}$ and $x, y \in \llbracket a, b \rrbracket \backslash \partial X$, $\left|\beta_{a}^{\times}(x, y)\right|=\left|\beta_{b}^{\times}(x, y)\right|=d^{\times}(x, y)$.

This extension $\beta^{\times}: \bar{X} \times(* \bar{X})^{2} \rightarrow \mathbb{R}$ will be called the horofunction in $\otimes \bar{X}$.

Proof We only need to prove (a) and ( $\left.a^{\prime}\right)$, then (b)-(d) follows from the properties of the original cocycle and (e) follows from Lemma 12(a) and Theorem $44(b)$.

(a) We have $x=\llbracket a, a^{\prime} ; s \rrbracket$ and $y=\llbracket b, b^{\prime} ; t \rrbracket$ as in 8.4. We saw in 8.4 that the formula

$$
\beta_{u}^{\times}(x, y):=\left\langle a \mid a^{\prime}\right\rangle_{u}+\left|s-\left\langle a, a^{\prime} \mid u\right\rangle\right|-\left\langle b \mid b^{\prime}\right\rangle_{u}-\left|t-\left\langle b, b^{\prime} \mid u\right\rangle\right|
$$

makes sense for the triples $(u, x, y) \in X \times(* \bar{X})^{2}$, and that for such triples $\beta_{u}^{\times}(x, y) \in \mathbb{R}$ and is Lipschitz in $u$. The inequality

$$
\begin{aligned}
& \left|\beta_{u}^{\times}\left(x^{\prime}, y^{\prime}\right)-\beta_{u}^{\times}(x, y)\right| \leq\left|\beta_{u}^{\times}\left(x^{\prime}, x\right)\right|+\left|\beta_{u}^{\times}\left(y^{\prime}, y\right)\right| \\
& \leq \sup _{u \in X}\left|\beta_{u}^{\times}\left(x^{\prime}, x\right)\right|+\sup _{u \in X}\left|\beta_{u}^{\times}\left(y^{\prime}, y\right)\right|=d^{\times}\left(x^{\prime}, x\right)+d^{\times}\left(y, y^{\prime}\right)
\end{aligned}
$$


shows that $\beta^{\times}$is also Lipschitz in $x$ and $y$, therefore in the three variables simultaneously.

(a') We want to extend $\beta^{\times}$to $\bar{X} \times(* \bar{X})^{2}$ continuously. Let $\bar{u} \in \partial X$. By [1, I $\S 8 \mathrm{~N}^{\circ} 5$, Theorem 1] it suffices to show the existence of the limit

$$
\lim \beta_{u}^{\times}(x, y) \quad \text { as } u \rightarrow \bar{u} \text { along } X
$$

in $\mathbb{R}$. First assume $\bar{u} \in \partial X \backslash\left\{a, a^{\prime}, b, b^{\prime}\right\}$, then by Theorem 35] and Theorem 36. as $u \rightarrow \bar{u}$ along $X$,

$$
\begin{aligned}
& \left\langle a, a^{\prime} \mid u\right\rangle \rightarrow\left\langle a, a^{\prime} \mid \bar{u}\right\rangle \in \mathbb{R}, \quad\left\langle b, b^{\prime} \mid u\right\rangle \rightarrow\left\langle b, b^{\prime} \mid \bar{u}\right\rangle \in \mathbb{R}, \\
& \left\langle a \mid a^{\prime}\right\rangle_{u}-\left\langle b \mid b^{\prime}\right\rangle_{u}=\left\langle a \mid a^{\prime}\right\rangle_{x_{0}}-\left\langle b \mid b^{\prime}\right\rangle_{x_{0}}+\left\langle a, b \mid u, x_{0}\right\rangle+\left\langle a^{\prime}, b^{\prime} \mid u, x_{0}\right\rangle \\
& \rightarrow\left\langle a \mid a^{\prime}\right\rangle_{x_{0}}-\left\langle b \mid b^{\prime}\right\rangle_{x_{0}}+\left\langle a, b \mid \bar{u}, x_{0}\right\rangle+\left\langle a^{\prime}, b^{\prime} \mid \bar{u}, x_{0}\right\rangle \in \mathbb{R},
\end{aligned}
$$

because under our assumptions there are no trivial $\partial X$-triples in the above terms. Hence by (51) the limit (52) exists in $\mathbb{R}$.

Now assume $\bar{u}=a \in \partial X \backslash\left\{a^{\prime}, b, b^{\prime}\right\}$, then as $u \rightarrow a$ along $X$,

$$
\left\langle b, b^{\prime} \mid u\right\rangle \rightarrow\left\langle b, b^{\prime} \mid a\right\rangle \in \mathbb{R} \quad \text { and } \quad\left\langle a, a^{\prime} \mid u\right\rangle \rightarrow-\infty,
$$

hence for $u$ sufficiently close to $a$,

$$
\begin{aligned}
& \left\langle a \mid a^{\prime}\right\rangle_{u}+\left|s-\left\langle a, a^{\prime} \mid u\right\rangle\right|-\left\langle b \mid b^{\prime}\right\rangle_{u}=\left\langle a \mid a^{\prime}\right\rangle_{u}+s-\left\langle a, a^{\prime} \mid u\right\rangle-\left\langle b \mid b^{\prime}\right\rangle_{u} \\
& =s+\left\langle a^{\prime}, b^{\prime} \mid u, x_{0}\right\rangle+\left\langle a^{\prime}, b \mid u, x_{0}\right\rangle \rightarrow s+\left\langle a^{\prime}, b^{\prime} \mid a, x_{0}\right\rangle+\left\langle a^{\prime}, b \mid a, x_{0}\right\rangle \in \mathbb{R} .
\end{aligned}
$$

(53) and (54) show that the limit (52) exists in $\mathbb{R}$. The similar argument works for each of the cases $\bar{u}=a^{\prime} \in \partial X \backslash\left\{a, b, b^{\prime}\right\}, \bar{u}=b \in \partial X \backslash\left\{a, a^{\prime}, b^{\prime}\right\}$, $\bar{u}=b^{\prime} \in \partial X \backslash\left\{a, a^{\prime}, b\right\}$.

Now assume $\bar{u}=a=b \in \partial X \backslash\left\{a^{\prime}, b^{\prime}\right\}$, then as $u \rightarrow a$ along $X$,

$$
\left\langle a, a^{\prime} \mid u\right\rangle \rightarrow-\infty \quad \text { and } \quad\left\langle b, b^{\prime} \mid u\right\rangle \rightarrow-\infty,
$$

hence for $u$ sufficiently close to $a$,

$$
\begin{aligned}
& \beta_{u}^{\times}(x, y)=\left\langle a \mid a^{\prime}\right\rangle_{u}+\left|s-\left\langle a, a^{\prime} \mid u\right\rangle\right|-\left\langle b \mid b^{\prime}\right\rangle_{u}-\left|t-\left\langle b, b^{\prime} \mid u\right\rangle\right| \\
& =\left\langle a \mid a^{\prime}\right\rangle_{u}+s-\left\langle a, a^{\prime} \mid u\right\rangle-\left\langle b \mid b^{\prime}\right\rangle_{u}-t+\left\langle b, b^{\prime} \mid u\right\rangle \\
& =\left\langle a^{\prime}, b^{\prime} \mid u, a\right\rangle+\left\langle a^{\prime}, b^{\prime} \mid u, x_{0}\right\rangle \rightarrow\left\langle a^{\prime}, b^{\prime} \mid a, x_{0}\right\rangle \in \mathbb{R} .
\end{aligned}
$$

The cases $\bar{u}=a=b^{\prime} \in \partial X \backslash\left\{a^{\prime}, b\right\}, \bar{u}=a^{\prime}=b \in \partial X \backslash\left\{a, b^{\prime}\right\}, \bar{u}=a^{\prime}=b^{\prime} \in$ $\partial X \backslash\{a, b\}$ are similar. Our condition (44) implies that there are no more cases to consider.

Of interest are special cases of Theorem 55 
- When $u \in X, x \in \llbracket a, u \rrbracket$ and $y \in \llbracket b, u \rrbracket, \beta_{u}^{\times}$becomes the usual distance cocycle: $\beta_{u}^{\times}(x, y)=d^{\times}(u, y)-d^{\times}(u, x)$. In particular, $\beta_{u}^{\times}(x, y)=\hat{d}(u, y)-$ $\hat{d}(u, x)$ for $u, x, y \in X$.

- When $u \in \partial X, \beta_{u}^{\times}$becomes a horofunction:

$\beta_{u}^{\times}(x, y)=\lim \left(d^{\times}(v, y)-d^{\times}(v, x)\right)$ as $v \rightarrow u$ along $X$. In particular, for $x, y \in X, \beta_{u}^{\times}(x, y)=\lim (\hat{d}(v, y)-\hat{d}(v, x))$ as $v \rightarrow u$ along $X$. Thus $\beta^{\times}$ satisfies the usual definition of a horofunction; the limits indeed exist.

For $x \in * \bar{X}, \mathcal{H}_{u}(x):=\left\{y \in * \bar{X} \mid \beta_{u}^{\times}(x, y)=0\right\}$ is the horosphere at $u$ containing $x$.

Lemma 56 Let $u, a, b \in \bar{X}$ and suppose that those of $u, a, b$ that lie in $\partial X$ are pairwise distinct. Then the four projections $\llbracket a, u|b \rrbracket, \llbracket b, u| a \rrbracket, \llbracket u, a \mid b \rrbracket$ and $\llbracket u, b \mid a \rrbracket$ lie on the same horosphere at $u$.

Proof The assumptions on $u, a, b$ and Theorem [35(fg) imply that $\langle a, u \mid b\rangle$, $\langle b, u \mid a\rangle,\langle u, a \mid b\rangle,\langle u, b \mid a\rangle$ are in $\mathbb{R}$, therefore all the four projections lie in $* \bar{X}$ and the horospheres containing them are indeed well-defined. If $u \in X$, using Definition 7.

$$
\begin{aligned}
& \beta_{u}^{\times}(\llbracket a, u|b \rrbracket, \llbracket b, u| a \rrbracket)=\beta_{u}^{\times}(\llbracket a, u ;\langle a, u \mid b\rangle \rrbracket, \llbracket b, u ;\langle b, u \mid a\rangle \rrbracket) \\
& =\langle b \mid u\rangle_{u}+|\langle b, u \mid u\rangle-\langle b, u \mid a\rangle|-\langle a \mid u\rangle_{u}-|\langle a, u \mid u\rangle-\langle a, u \mid b\rangle| \\
& =|\langle b, u \mid u, a\rangle|-|\langle a, u \mid u, b\rangle|=\langle b \mid a\rangle_{u}-\langle a \mid b\rangle_{u}=0,
\end{aligned}
$$

i.e. $\llbracket a, u \mid b \rrbracket$ and $\llbracket b, u \mid a \rrbracket$ lie on the same horosphere at $u$. This extends by continuity (Theorem $[55$ (a)) to the case $u \in \bar{X}$. The rest of lemma follows from the $\mathbb{Z}_{2}$-invariance of $\beta^{\times}$:

$$
\beta_{u}^{\times}(\llbracket a, u|b \rrbracket, \llbracket u, a| b \rrbracket)=\beta_{u}^{\times}\left(\llbracket a, u|b \rrbracket, \llbracket a, u| b \rrbracket^{\star}\right)=\beta_{u}^{\times}(\llbracket a, u|b \rrbracket, \llbracket a, u| b \rrbracket)=0,
$$

and similarly for $\llbracket u, b \mid a \rrbracket$.

\section{Synchronous exponential convergence of lines in $\otimes \bar{X}$}

Recall from 2.10 and 8.1 that $\llbracket b, c ; \cdot \rrbracket_{a}$ is the isometric reparametrization of $\llbracket b, c \rrbracket$ whose origin $\llbracket b, c ; 0 \rrbracket_{a}$ is the projection of $a$ to $\llbracket b, c \rrbracket$.

Theorem 57 (Exponential convergence in $d^{\times}$) Let $X$ be a hyperbolic complex. There exist $N \in[0, \infty)$ and $\lambda \in(1 / e, 1)$ depending only on $X$ such that for all $t \in \mathbb{R}$ and $a, b, c \in \bar{X}$,

$$
d^{\times}\left(\llbracket b, c ; t \rrbracket_{a}, \llbracket a, c ; t \rrbracket_{b}\right) \leq N \lambda^{t} .
$$


This theorem provides

- an upper exponential bound that is independent of the choice of $a, b, c$;

- a synchronous exponential convergence: it is easy to see from Lemma 56 that at each time $t \geq 0, \llbracket b, c ; t \rrbracket_{a}$ and $\llbracket a, c ; t \rrbracket_{b}$ lie on the same horosphere centered at $c$;

- the place where the exponential convergence starts occurring, namely the projections $\llbracket b, c \mid a \rrbracket$ and $\llbracket a, c \mid b \rrbracket$ corresponding to $t=0$.

Note also that $t$ is not assumed to be appropriate for $\llbracket b, c \rrbracket_{a}$ or $\llbracket a, c \rrbracket_{b}$.

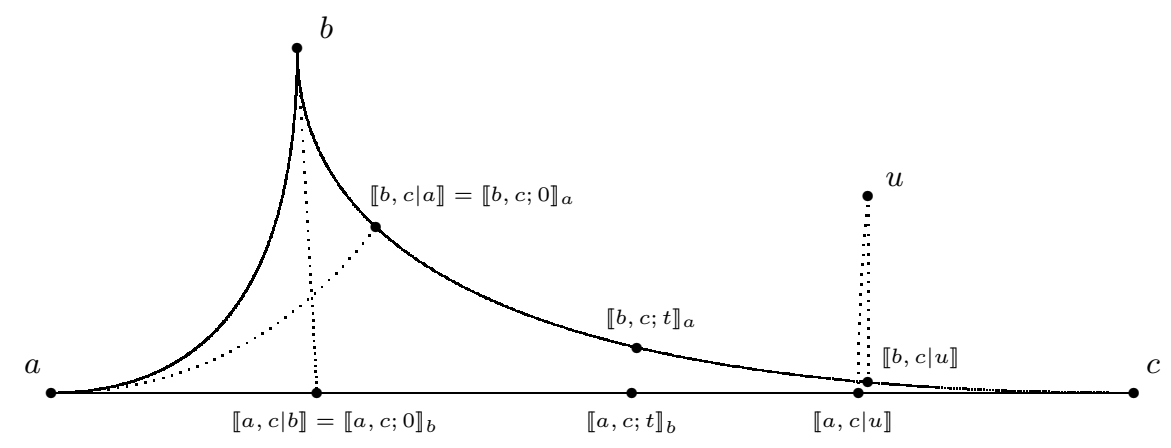

Figure 6: Synchronous exponential convergence of lines

Proof First note that if $a=b \in \partial X$ or $a=c \in \partial X$ or $b=c \in X$, then $\llbracket b, c ; t \rrbracket_{a}=\llbracket a, c ; t \rrbracket_{b} \in \partial X$ and the lemma obviously follows (here we view $d^{\times}$as a generalized pseudometric in $\otimes \bar{X}$ ). So from now we will assume that those of $a, b, c$ that lie in $\partial X$ are pairwise distinct.

Let $T \in[0, \infty)$ and $\lambda \in[0,1)$ be the constants from Lemma 38. Increase $\lambda$ if needed so that $\lambda \in(1 / e, 1)$. First we will show that

$$
d^{\times}\left(\llbracket b, c ; t \rrbracket_{a}, \llbracket a, c ; t \rrbracket_{b}\right) \leq 2 \lambda^{t} \quad \text { for all } t \in[T, \infty),
$$

i.e. that the lemma holds under the additional assumption $t \geq T$. By the definition of $d^{\times}$, (55) is equivalent to

$$
\left|\beta_{u}^{\times}\left(\llbracket b, c ; t \rrbracket_{a}, \llbracket a, c ; t \rrbracket_{b}\right)\right| \leq 2 \lambda^{t} \quad \text { for all } u \in X, t \in[T, \infty) .
$$

By the definition of $\beta^{\times}$,

$$
\begin{aligned}
& \beta_{u}^{\times}\left(\llbracket b, c ; t \rrbracket_{a}, \llbracket a, c ; t \rrbracket_{b}\right)=\beta_{u}^{\times}(\llbracket b, c ; t+\langle b, c \mid a\rangle \rrbracket, \llbracket a, c ; t+\langle a, c \mid b\rangle \rrbracket) \\
& =\langle b \mid c\rangle_{u}+|t+\langle b, c \mid a\rangle-\langle b, c \mid u\rangle|-\langle a \mid c\rangle_{u}-|t+\langle a, c \mid b\rangle-\langle a, c \mid u\rangle| \\
& =-\langle u, c \mid a, b\rangle+|t-\langle u, a \mid b, c\rangle|-|t-\langle u, b \mid a, c\rangle| .
\end{aligned}
$$


This equality will be used in the computations below. Pick an arbitrary $u \in X$.

If $\max \{\langle u, a \mid b, c\rangle,\langle u, b \mid a, c\rangle\} \leq t$, then

$\left|\beta_{u}^{\times}\left(\llbracket b, c ; t \rrbracket_{a}, \llbracket a, c ; t \rrbracket_{b}\right)\right|=|-\langle u, c \mid a, b\rangle+(t-\langle u, a \mid b, c\rangle)-(t-\langle u, b \mid a, c\rangle)|=0 \leq 2 \lambda^{t}$.

If $\max \{\langle u, a \mid b, c\rangle,\langle u, b \mid a, c\rangle\} \geq t$ (see Figure [6), then $\max \{\langle u, a \mid b, c\rangle,\langle u, b \mid a, c\rangle\} \geq T$ and by Proposition 38.

$$
\begin{aligned}
& \left|\beta_{u}^{\times}\left(\llbracket b, c ; t \rrbracket_{a}, \llbracket a, c ; t \rrbracket_{b}\right)\right| \leq|\langle u, c \mid a, b\rangle|+|| t-\langle u, a \mid b, c\rangle|-| t-\langle u, b \mid a, c\rangle|| \\
& \leq|\langle u, c \mid a, b\rangle|+|\langle u, b \mid a, c\rangle-\langle u, a \mid b, c\rangle|=2|\langle u, c \mid a, b\rangle| \\
& \leq 2 \lambda^{\max \{\langle u, a \mid b, c\rangle,\langle u, b \mid a, c\rangle\}} \leq 2 \lambda^{t} .
\end{aligned}
$$

This proves (55]). By calculus, there exists $N^{\prime} \in[0, \infty)$ depending only on $T$ and $\lambda$ such that

$$
2\left(\lambda^{t}+T-t\right) \leq N^{\prime} \lambda^{t} \quad \text { for all } t \leq T .
$$

Let $N:=\max \left\{2, N^{\prime}\right\}$, then (55) implies that the lemma holds for all $t \geq T$. It remains to prove the lemma under the assumption $t \leq T$. By Theorem 44(b), (55) and since $\llbracket b, c ; \cdot \rrbracket_{a}$ and $\llbracket a, c ; \cdot \rrbracket_{b}$ are non-expanding,

$$
\begin{aligned}
& d^{\times}\left(\llbracket b, c ; t \rrbracket_{a}, \llbracket a, c ; t \rrbracket_{b}\right) \\
& \leq d^{\times}\left(\llbracket b, c ; t \rrbracket_{a}, \llbracket b, c ; T \rrbracket_{a}\right)+d^{\times}\left(\llbracket b, c ; T \rrbracket_{a}, \llbracket a, c ; T \rrbracket_{b}\right)+d^{\times}\left(\llbracket a, c ; T \rrbracket_{b}, \llbracket a, c ; t \rrbracket_{b}\right) \\
& \leq d^{\times}\left(\llbracket b, c ; T \rrbracket_{a}, \llbracket a, c ; T \rrbracket_{b}\right)+2(T-t) \leq 2 \lambda^{T}+2(T-t) \\
& \leq 2\left(\lambda^{t}+T-t\right) \leq N^{\prime} \lambda^{t} \leq N \lambda^{t} .
\end{aligned}
$$

\section{Translation length}

For a hyperbolic complex $X$, we define the translation length $\hat{l}(g)$ of $g \in \operatorname{Isom}(X)$ via the metric $\hat{d}$, that is

$$
\hat{l}(g):=\lim _{n \rightarrow \infty} \hat{d}\left(x, g^{n} x\right) / n
$$

for some point $x \in X$. By the triangle inequality, $\hat{l}(g)$ is independent of the choice of $x$. In particular $\hat{l}(g)=\hat{l}\left(g^{\prime}\right)$ if $g$ and $g^{\prime}$ are conjugate in $\operatorname{Isom}(X)$, so $\hat{l}$ can be viewed as the length spectrum of $\operatorname{Isom}(X)$, i.e. a function on the conjugacy classes. This concept is of interest from the geometric point of view, for example Otal [33] and Croke [14] showed that each negatively curved metric on a surface is determined up to an isometry by its length spectrum.

An isometry of $X$ is called elliptic if it has a bounded orbit in $X$; this implies that each orbit is bounded. An isometry $g$ is called hyperbolic if its translation 
length (say with respect to the word metric) is positive; this implies that $g$ fixes exactly 2 points at infinity. We denote $g_{-}$the repelling point and $g_{+}$the attracting point. All isometries of a hyperbolic complex $X$ are either elliptic or hyperbolic.

Using $\hat{d}$ instead of the word metric enables us to express translation length in terms of the double difference as follows ( $\mathrm{cf}[28$ for the CAT $(-1)$ case).

Proposition $58 \hat{l}(g)$ in (56) is a well-defined real number. Moreover, for any hyperbolic $g \in \operatorname{Isom}(X)$ and any $x \in \bar{X} \backslash\left\{g_{-}, g_{+}\right\}$,

$$
\hat{l}(g)=\left\langle g_{-}, g_{+} \mid g x, x\right\rangle=\ln \| g_{-}, g_{+} \mid g x, x \rrbracket .
$$

In particular, $\left\langle g_{-}, g_{+} \mid g x, x\right\rangle$ and $\left\|g_{-}, g_{+} \mid g x, x\right\|$ are independent of the choice of $x$.

Proof If $g$ is elliptic then the orbit of any $x \in X$ is bounded, hence $\hat{l}(g)=0$. If $g$ is hyperbolic, pick a geodesic $\gamma$ from $g_{-}$to $g_{+}$and $y \in \gamma$. All geodesics $g^{n} \gamma$ are $\delta$-close to $\gamma$, so for each $n$ we choose a point $y_{n} \in \gamma$ which is $\delta$ close to $g^{n} y$. By [31, Proposition 10(b)] and the definition of $\hat{d}$, there is a constant $C \in[0, \infty)$ such that for all $n \geq 0$ and all $v \in \gamma$ between $g^{n} y$ and $g_{+}$, $\left|\hat{d}(y, v)-\hat{d}\left(y_{n}, v\right)-\hat{d}\left(y, y_{n}\right)\right| \leq C$. Since $g^{n} y$ and $y_{n}$ are $\delta$-close, we can also assume $\left|\hat{d}(y, v)-\hat{d}\left(y, g^{n} y\right)-\hat{d}\left(g^{n} y, v\right)\right| \leq C$. Similarly, for all $u \in \gamma$ between $g_{-}$ and $y,\left|\hat{d}\left(u, g^{n} y\right)-\hat{d}(u, y)-\hat{d}\left(y, g^{n} y\right)\right| \leq C$. Combining the two inequalities,

$$
\begin{aligned}
& \left|\left\langle u, v \mid g^{n} y, y\right\rangle-\hat{d}\left(y, g^{n} y\right)\right| \leq\left|\hat{d}(y, v)-\hat{d}\left(y, g^{n} y\right)-\hat{d}\left(g^{n} y, v\right)\right| / 2 \\
& +\left|\hat{d}\left(u, g^{n} y\right)-\hat{d}(u, y)-\hat{d}\left(y, g^{n} y\right)\right| / 2 \leq C / 2+C / 2=C .
\end{aligned}
$$

By continuity of double difference as $u \rightarrow g_{-}$and $v \rightarrow g_{+}$,

$$
\left|\left\langle g_{-}, g_{+} \mid g^{n} y, y\right\rangle-\hat{d}\left(y, g^{n} y\right)\right| \leq C .
$$

By the invariance of double difference under $g$,

$$
\begin{aligned}
& \left\langle g_{-}, g_{+} \mid g y, y\right\rangle=\left\langle g_{-}, g_{+} \mid g^{2} y, g y\right\rangle=\ldots=\left\langle g_{-}, g_{+} \mid g^{n} y, g^{n-1} y\right\rangle, \quad \text { hence } \\
& \left\langle g_{-}, g_{+} \mid g^{n} y, y\right\rangle=\left\langle g_{-}, g_{+} \mid g y, y\right\rangle+\ldots+\left\langle g_{-}, g_{+} \mid g^{n} y, g^{n-1} y\right\rangle=n\left\langle g_{-}, g_{+} \mid g y, y\right\rangle,
\end{aligned}
$$

and (57) rewrites as $\left|\left\langle g_{-}, g_{+} \mid g y, y\right\rangle-\hat{d}\left(y, g^{n} y\right) / n\right| \leq C / n$. Therefore $\lim _{n \rightarrow \infty} \hat{d}\left(y, g^{n} y\right) / n$ exists and equals $\left\langle g_{-}, g_{+} \mid g y, y\right\rangle$, so $\hat{l}(g)$ is well-defined and $\hat{l}(g)=\left\langle g_{-}, g_{+} \mid g y, y\right\rangle$.

For any $x \in \bar{X} \backslash\left\{g_{-}, g_{+}\right\}$, by $g$-invariance, $\left\langle g_{-}, g_{+} \mid g x, g y\right\rangle=\left\langle g_{-}, g_{+} \mid x, y\right\rangle$, hence

$$
\begin{aligned}
& \left\langle g_{-}, g_{+} \mid g x, x\right\rangle=\left\langle g_{-}, g_{+} \mid g x, g y\right\rangle+\left\langle g_{-}, g_{+} \mid g y, y\right\rangle+\left\langle g_{-}, g_{+} \mid y, x\right\rangle \\
& =\left\langle g_{-}, g_{+} \mid g y, y\right\rangle=\hat{l}(g) .
\end{aligned}
$$




\section{The geodesic flow of a hyperbolic complex}

Let $X$ be a hyperbolic complex. As an example, the reader might think of a Cayley graph of a hyperbolic group, of the group itself. Recall from 6.1 that $X$ admits a nice canonical metric $\hat{d}$, and we use it to define the (generalized) metric $d_{*}$ on the symmetric join $\otimes \bar{X}$. A part of the above symmetric join construction is the flow space of $X$,

$$
\mathcal{F}(X):=*(\partial X):=\circledast(\partial X) \backslash \partial X \subseteq \otimes \bar{X} .
$$

As a set, $\mathcal{F}(X)$ is a disjoint union of open lines connecting disjoint ordered pairs of points at infinity. We will use the same notation $d_{*}$ for the restriction of $d_{*}$ to $\mathcal{F}(X) .\left(\mathcal{F}(X), d_{*}\right)$ plays the role of the total space of the unit tangent bundle on $X$ (though no bundle map is there), and it is canonically defined for any hyperbolic complex $X$.

Proposition 59 (a) For $a, b \in \partial X, \llbracket a, b ; \cdot \rrbracket^{\prime}=\llbracket a, b ; \cdot \rrbracket$.

(b) The restrictions of $d^{\times}$and $d_{*}$ to each line in $\mathcal{F}(X)$ coincide with the original metric on the line.

(c) For each $x \in \mathcal{F}(X)$, the orbit map $\mathbb{R} \rightarrow\left(\mathcal{F}(X), d_{*}\right), r \mapsto r^{+} x$, is an isometry onto the $\mathbb{R}$-orbit containing $x$.

Proof (a) Since $a, b \in \partial X, \llbracket a, b \rrbracket$ is a copy of $[-\infty, \infty]$ and $\llbracket a, b ; \cdot \rrbracket:[-\infty, \infty] \rightarrow \llbracket a, b \rrbracket$ is the identity map. By definitions in 2.2

$$
\llbracket a, b ; t \rrbracket^{\prime}=\int_{-\infty}^{\infty} \llbracket a, b ; r+t \rrbracket \frac{e^{-|r|}}{2} d r=\int_{-\infty}^{\infty}(r+t) \frac{e^{-|r|}}{2} d r=t=\llbracket a, b ; t \rrbracket .
$$

(b) By (a) and Theorem 44(b),

$$
\begin{aligned}
& d_{*}\left(\llbracket a, b ; s \rrbracket^{\prime}, \llbracket a, b ; t \rrbracket^{\prime}\right)=\int_{-\infty}^{\infty} d^{\times}\left(\llbracket a, b ; r+s \rrbracket^{\prime}, \llbracket a, b ; r+t \rrbracket^{\prime}\right) \frac{e^{-|r|}}{2} d r \\
& =\int_{-\infty}^{\infty} d^{\times}(\llbracket a, b ; r+s \rrbracket, \llbracket a, b ; r+t \rrbracket) \frac{e^{-|r|}}{2} d r=\int_{-\infty}^{\infty}|t-s| \frac{e^{-|r|}}{2} d r=|t-s| \\
& =d^{\times}(\llbracket a, b ; s \rrbracket, \llbracket a, b ; t \rrbracket)=d^{\times}\left(\llbracket a, b ; s \rrbracket^{\prime}, \llbracket a, b ; t \rrbracket^{\prime}\right) .
\end{aligned}
$$

(c) Let $x=\llbracket a, b ; t \rrbracket^{\prime}, a, b \in \partial X$, then by (a), (b) and Theorem 44(b),

$$
\begin{aligned}
& d_{*}\left(r_{1}^{+} x, r_{2}^{+} x\right)=d_{*}\left(\llbracket a, b ; r_{1}+t \rrbracket^{\prime}, \llbracket a, b ; r_{2}+t \rrbracket^{\prime}\right) \\
& \quad=d^{\times}\left(\llbracket a, b ; r_{1}+t \rrbracket, \llbracket a, b ; r_{2}+t \rrbracket\right)=\left|r_{2}-r_{1}\right| .
\end{aligned}
$$

Let $\partial^{2} X:=\left\{(a, b) \in(\partial X)^{2} \mid a \neq b\right\}$. Recall from 1.3 that ${ }^{x+}$ isometry is quasiisometry, and ${ }^{+}$isometry is "quasiisometry with multiplicative constant 1 ". We summarize the properties of the flow space (cf [27, 8.3.C]). 
Theorem 60 (Geodesic flow of $X$ ) Let $X$ be a hyperbolic complex, $d$ the word metric and $\hat{d}=\hat{d}_{X}$ the canonical metric as in 6.1. Then there is a metric space $\left(\mathcal{F}(X), d_{*}\right)$ canonically associated to $X$ with the following properties.

(a) $\left(\mathcal{F}(X), d_{*}\right)$ is homeomorphic to $\partial^{2} X \times \mathbb{R}$.

(b) $\left(\mathcal{F}(X), d_{*}\right)$ is proper.

(c) If $\operatorname{Isom}(X)$ has a cobounded orbit in $X$, for example if $X$ admits a cocompact isometric action, then $\left(\mathcal{F}(X), d_{*}\right)$ is ${ }^{+}$isometric to $(X, \hat{d})$ and $\times+$ isometric to $(X, d)$.

(d) There is a canonical isometric action of $\operatorname{Isom}(X)$ on $\left(\mathcal{F}(X), d_{*}\right)$.

(e) There is a canonical free $\mathbb{R}$-action $(r, x) \mapsto r^{+} x$ on $\left(\mathcal{F}(X), d_{*}\right)$ by biLipschitz homeomorphisms which commutes with the $\operatorname{Isom}(X)$-action. For each $x \in \mathcal{F}(X)$ the orbit map $\mathbb{R} \rightarrow\left(\mathcal{F}(X), d_{*}\right), r \mapsto r^{+} x$, is an isometry onto the $\mathbb{R}$-orbit containing $x$. In particular, $\mathbb{R}$ acts by isometries on each $\mathbb{R}$-orbit in $\mathcal{F}(X)$, in the standard way.

(f) There is a canonical free $\mathbb{Z}_{2}$-action on $\left(\mathcal{F}(X), d_{*}\right), x \mapsto x^{\star}$, by isometries which commutes with the $\operatorname{Isom}(X)$-action and anticommutes with the $\mathbb{R}$-action. It moves every point a uniformly bounded distance and fixes $\partial X$ pointwise.

(g) There exists a horofunction $\beta_{u}^{\times}: \mathcal{F}(X)^{2} \rightarrow \mathbb{R}$ which is continuous in three variables $(u, x, y) \in \bar{X} \times \mathcal{F}(X)^{2}$ and satisfies

(i) $\left(\mathbb{Z}_{2}\right.$-invariance) $\beta_{u}^{\times}(x, y)=\beta_{u}^{\times}\left(x^{\star}, y\right)=\beta_{u}^{\times}\left(x, y^{\star}\right)$.

(ii) (cocycle condition) $\beta_{u}^{\times}(x, y)+\beta_{u}^{\times}(y, z)=\beta_{u}^{\times}(x, z)$.

(iii) $\left(\operatorname{Isom}(X)\right.$-invariance) $\beta_{g u}^{\times}(g x, g y)=\beta_{u}^{\times}(x, y)$ for $g \in \operatorname{Isom}(X)$.

(iv) (isometry on $\mathbb{R}$-orbits) For each $\mathbb{R}$-orbit $\rrbracket a, b \llbracket$ in $\mathcal{F}(X)$ and

$$
x, y \in \rrbracket a, b \llbracket, \quad\left|\beta_{a}^{\times}(x, y)\right|=\left|\beta_{b}^{\times}(x, y)\right|=d_{*}(x, y) .
$$

(h) (exponential convergence in $d_{*}$ ) There exist $M \in[0, \infty)$ and $\lambda \in[0,1)$ depending only on $X$ with the following property. For all $a, b, c \in \partial X$, take the isometric parametrizations $\rrbracket a, c ; \cdot \llbracket b: \mathbb{R} \rightarrow \rrbracket a, c \llbracket$ and $\rrbracket b, c ; \cdot \llbracket a: \mathbb{R} \rightarrow$ $\rrbracket b, c \llbracket$ of the $\mathbb{R}$-orbits $\rrbracket a, c \llbracket$ and $\rrbracket b, c \llbracket$ as described in 8.1 Then $\llbracket b, c ; t \rrbracket_{a}$ and $\llbracket a, c ; t \rrbracket_{b}$ lie on the same horosphere at $c$ and

$$
d_{*}\left(\llbracket a, c ; t \rrbracket_{b}, \llbracket b, c ; t \rrbracket_{a}\right) \leq M \lambda^{t} .
$$

Similarly for any pair of lines among $\rrbracket a, c \llbracket, \rrbracket b, c \llbracket, \rrbracket c, a \llbracket, \rrbracket c, b \llbracket$.

(i) Let $\hat{l}$ be the translation length in $X$ defined in section 12, Then for any hyperbolic $g \in \operatorname{Isom}(X)$ and any $y \in \mathcal{F}(X)$,

$$
\hat{l}(g)=\lim _{n \rightarrow \infty} d_{*}\left(y, g^{n} y\right) / n=\inf \left\{d_{*}(y, g y) \mid y \in \mathcal{F}(X)\right\} .
$$

Moreover, if $z \in \rrbracket g_{-}, g_{+} \llbracket$ then $d_{*}(z, g z)=\hat{l}(g)$. 
Remark Note that the symmetric join $\otimes \bar{X}$ from section 8 gives even more structure: both $X$ and $\mathcal{F}(X)$ isometrically embed into $\oplus \bar{X}$, so $\oplus \bar{X}$ can be thought of as a "filling" between $X$ and $\mathcal{F}(X)$. This is a metric analogue of the following geometric situation: if $Y$ is a smooth manifold, then the space $B_{1} Y$ of tangent vectors of length at most 1 contains both the unit sphere bundle $S_{1} Y$ and $Y$, as the 0 -section, so $B_{1} Y$ is a "filling" between $S_{1} Y$ and $Y$.

Proof of Theorem 60 (a) The homeomorphism

$$
\rrbracket \cdot, \cdot ; \cdot \mathbb{I}^{\prime}:\left(\bar{X}^{2} \backslash \bar{\Delta}\right) \times \mathbb{R} \rightarrow\left(x_{0}^{*} \bar{X}, d_{*}\right)
$$

from Proposition 48 maps $\partial^{2} X \times \mathbb{R}$ onto $\mathcal{F}(X)$.

(b) The same proof as in Proposition 54 with $\left(\mathcal{F}(X), d_{*}\right)$ instead of $\left(* \bar{X}, d_{*}\right)$.

(c) The proof of ${ }^{+}$isometry between $\left(\mathcal{F}(X), d_{*}\right)$ and $(X, \hat{d})$ is the same as in Proposition 43. The map $\psi: \mathcal{F}(X) \rightarrow X$ is not surjective, but the assumptions imply that it has cobounded image in $X$; this is sufficient to run the argument. The ${ }^{x+}$ isometry of $\left(\mathcal{F}(X), d_{*}\right)$ and $(X, d)$ follows since $d$ and $\hat{d}$ are ${ }^{x+}$ equivalent by Theorem $32(\mathrm{a})$. (d)-(g) were proved in earlier sections.

(h) Let $\lambda \in(1 / e, 1)$ and $N \in[0, \infty)$ be the constants from Theorem 57. Using the identity $\llbracket \cdot, \cdot ; \cdot \rrbracket^{\prime}=\llbracket \cdot, \cdot ; \cdot \rrbracket$ in $\mathcal{F}(X)$ and the definition of $d_{*}$,

$$
\begin{aligned}
& d_{*}\left(\llbracket a, c ; t \rrbracket_{b}, \llbracket b, c ; t \rrbracket_{a}\right)=d_{*}(\llbracket a, c ; t+\langle a, c \mid b\rangle \rrbracket, \llbracket b, c ; t+\langle b, c \mid a\rangle \rrbracket) \\
& =\int_{-\infty}^{\infty} d^{\times}(\llbracket a, c ; t+\langle a, c \mid b\rangle \rrbracket, \llbracket b, c ; t+\langle b, c \mid a\rangle \rrbracket) \frac{e^{-|r|}}{2} d r \\
& =\int_{-\infty}^{\infty} d^{\times}\left(\llbracket a, c ; r+t \rrbracket_{b}, \llbracket b, c ; r+t \rrbracket_{a}\right) \frac{e^{-|r|}}{2} d r \\
& \leq \int_{-\infty}^{\infty} N \lambda^{r+t} \frac{e^{-|r|}}{2} d r=\frac{N \lambda^{t}}{1-(\ln \lambda)^{2}}
\end{aligned}
$$

so we denote $M:=N /\left(1-(\ln \lambda)^{2}\right)$.

(i) $g \in \operatorname{Isom}(X)$ is an isometry of $\left(* \bar{X}, d_{*}\right)$. For any $y \in * \bar{X}$, the limit $\lim _{n \rightarrow \infty} d_{*}\left(y, g^{n} y\right) / n$ exists, and it is independent of $y$ (see [4, II.6.6(1)]). Since $d_{*}$ coincides with $\hat{d}$ on $X$, for any $x \in X$ we have

$$
\lim _{n \rightarrow \infty} d_{*}\left(y, g^{n} y\right) / n=\lim _{n \rightarrow \infty} d_{*}\left(x, g^{n} x\right) / n=\lim _{n \rightarrow \infty} \hat{d}\left(x, g^{n} x\right) / n=\hat{l}(g) .
$$

This proves the first equality. For all $y \in * \bar{X}$,

$$
d_{*}\left(y, g^{n} y\right) / n \leq\left(d_{*}(y, g y)+\ldots+d_{*}\left(g^{n-1} y, g^{n} y\right)\right) / n=d_{*}(y, g y),
$$


hence $\hat{l}(g)=\lim _{n \rightarrow \infty} d_{*}\left(y, g^{n} y\right) / n \leq d_{*}(y, g y)$, so

$$
\hat{l}(g) \leq \inf \left\{d_{*}(y, g y) \mid y \in \mathcal{F}(X)\right\} .
$$

Let $g_{-}, g_{+} \in \partial X$ be the fixed points of $g$ and $z \in \rrbracket g_{-}, g_{+} \llbracket$, then by the last two properties in $(\mathrm{g})$,

$$
\begin{aligned}
& d_{*}(z, g z)=\left|\beta_{g_{+}}^{\times}(z, g z)\right|=\left|\beta_{g_{+}}^{\times}(z, g z)+\ldots+\beta_{g_{+}}^{\times}\left(g^{n-1} z, g^{n} z\right)\right| / n \\
& =\left|\beta_{g_{+}}^{\times}\left(z, g^{n} z\right)\right| / n=d_{*}\left(z, g^{n} z\right) / n \text { for any } n,
\end{aligned}
$$

therefore $d_{*}(z, g z)=\hat{l}(g)$ and (58) becomes an equality.

\section{Asymmetric join, the Borel conjecture and gen- eral remarks}

\subsection{The definition of asymmetric join}

If $\left(Y_{1}, d_{1}\right)$ and $\left(Y_{2}, d_{2}\right)$ are metric spaces, let $X:=Y_{1} \sqcup Y_{2}$ and pick a metric $d$ on $X$ which induces the original topologies on $Y_{1}$ and $Y_{2}$. Then $d$ canonically extends to the metric $d_{*}=\circledast d$ on $\otimes X$ as in 3.3 and we define the asymmetric join of $Y_{1}$ and $Y_{2}$ to be the subspace $Y_{1} \otimes Y_{2} \subseteq \otimes X$ which is the union of all lines in $\otimes X$ going from points in $Y_{1}$ to points in $Y_{2}$, with the restricted metric $d_{*}$.

For arbitrary $Y_{1}$ and $Y_{2}$, there is a choice involved in defining $d$ on the union $Y_{1} \sqcup Y_{2}$, but if $Y_{1}$ and $Y_{2}$ are pointed metric spaces with isometric actions by the same group $G$, then $d$ and $d_{*}$ can be defined canonically, as described below in 14.2. First let us mention some important examples when this situation arises:

(1) Under the hypotheses of the Borel conjecture (in the PL setting), $M_{1}$ and $M_{2}$ are closed triangulated manifolds with the same fundamental group $\Gamma$. Consider the universal cover $Y_{i}:=\widetilde{M}_{i}$ with any $\Gamma$-invariant metrics $d_{i}$ induced from 0 -skeleton $(\operatorname{cf}[5.2(3))$, and let $G:=\Gamma$. If $\Gamma$ is hyperbolic, put the canonical metrics $\hat{d}_{Y_{i}}$ on $Y_{i}(\operatorname{cf} 6.3(3))$.

(2) If $M_{1}$ and $M_{2}$ are smooth manifolds with the same fundamental group $\Gamma$, let $d_{i}$ be the $\Gamma$-invariant intrinsic metric on the universal cover $Y_{i}:=\widetilde{M}_{i}$, and $G:=\Gamma$.

(3) For arbitrary metric complexes $\left(Y_{1}, d_{1}\right)$ and $\left(Y_{2}, d_{2}\right)$ one could just let $G$ be the trivial group. 


\subsection{A metric on $Y_{1} \sqcup Y_{2}$}

Suppose that $\left(Y_{1}, d_{1}\right)$ and $\left(Y_{2}, d_{2}\right)$ are metric spaces with isometric actions by the same group $G$. Let $X:=Y_{1} \sqcup Y_{2}$. Pick basepoints $y_{1} \in Y_{1}$ and $y_{2} \in Y_{2}$. A pair $(y, z)$ of points in $X$ is called admissible if

(a) both $y$ and $z$ belong to the same $Y_{i}$, or

(b) $y=g y_{1}$ and $z=g y_{2}$ for some $g \in G$, or

(c) vise versa, $y=g y_{2}$ and $z=g y_{1}$ for some $g \in G$.

The length of an admissible pair $(y, z), l(y, z)$, is defined to be $d_{i}(y, z)$ in case $(a)$ and 1 in cases $(b)$ and $(c)$. A finite sequence $x_{1}, \ldots, x_{n}$ of points in $X$ is called admissible if each consecutive pair $\left(x_{j}, x_{j+1}\right)$ is admissible. The length of an admissible sequence, $l\left(x_{1}, \ldots, x_{n}\right)$, is $\sum_{j=1}^{n-1} l\left(x_{j}, x_{j+1}\right)$. We define a metric $d$ on $X$ by

$$
d(a, b):=\inf l\left(x_{1}, \ldots, x_{n}\right)
$$

over all admissible sequences $x_{1}, \ldots, x_{n}$ in $X$ with $x_{1}=a$ and $x_{n}=b$. If $a, b \in Y_{i}$ and $d_{i}(a, b) \leq 1$, then $d(a, b)=d_{i}(a, b)$, i.e. $d$ and $d_{i}$ locally coincide on $Y_{i}$. Therefore $d$ induces the original topologies on $Y_{1}$ and $Y_{2}$. If $G$ has a cobounded orbit in $Y_{i}$, for example if the $G$-action on $Y_{i}$ is cocompact, then the embedding $\left(Y_{i}, d_{i}\right) \hookrightarrow(X, d)$ is a quasiisometry.

The above definition of $d$ on $X=Y_{1} \sqcup Y_{2}$ makes the construction of the asymmetric join $Y_{1} \otimes Y_{2}$ and of the metric $d_{*}=\otimes d$ canonical. In the case (1) above, if $M_{1}$ and $M_{2}$ are closed manifolds and $\pi_{1}\left(M_{1}\right)=\pi_{1}\left(M_{2}\right)$ is hyperbolic, then the join $\bar{Y}_{1} \otimes \bar{Y}_{2}$ of the compactifications is also well-defined, equipped with the generalized metric $d_{*}$. This allows for the use of both local and global structures of the manifolds.

The asymmetric join is another example of a metric join.

\subsection{Various model spaces for hyperbolic groups}

Given a hyperbolic group $\Gamma$, one can take $X$ to be either

- the group $\Gamma$ itself,

- or a Cayley graph of $\Gamma$,

- or any other simplicial complex on which $\Gamma$ acts (say, cocompactly). 
We can put the metric $\hat{d}$ on $X$ as in 6.1 then the constructions of this paper provide many model spaces, each equipped with the (generalized) metric $d_{*}$ and a $\Gamma$-action, for example $\otimes X, \oplus \bar{X}, \mathcal{F}(X), X \otimes X, X \otimes \bar{X}, \bar{X} \otimes \bar{X}$. The symmetric and asymmetric join functors can be iterated, so for example, $\otimes((\otimes(X \otimes \bar{X})) \otimes(* X))$ is a legitimate model space with an isometric $\Gamma$-action.

\subsection{More general metric spaces}

We started with an arbitrary metric space $(X, d)$ and defined $d^{\times}$and $d_{*}$ on $\otimes X$. When $X$ is a hyperbolic complex, the metric $\hat{d}$ on $X$ was used to define $d^{\times}$and $d_{*}$ on $\otimes \bar{X}$ (with infinite values allowed). The metric $\hat{d}$ was used because of its strong properties at infinity. The definition of $d^{\times}$and $d_{*}$ can be carried out if one starts with a more general hyperbolic metric space $(X, \hat{d})$, where $\hat{d}$ satisfies the properties of 6.6 through 6.8. For example this would work for $C A T(-1)$ spaces.

\subsection{The sweep-out and the Borel conjecture}

Each line $\llbracket a, b \rrbracket$ in $Y_{1} \otimes Y_{2}$ with $a \in Y_{1}$ and $b \in Y_{2}$, is given the canonical parametrization $\llbracket a, b ; \cdot \rrbracket^{\prime}: \overline{\mathbb{R}} \rightarrow \llbracket a, b \rrbracket$. Each $t \in \overline{\mathbb{R}}$ gives the point $\llbracket a, b ; t \rrbracket^{\prime}$ on each line $\llbracket a, b \rrbracket$ in $Y_{1} \circledast Y_{2}$; the union $S_{t}$ of these points for a fixed $t$ will be called a slice, and the set of all slices is the sweep-out from $Y_{1}$ to $Y_{2}$. For each $t \in \mathbb{R}, S_{t}$ is homeomorphic to $Y_{1} \times Y_{2}$ (this follows from Proposition 201), but $S_{t}$ converges to $Y_{1}$ as $t \rightarrow-\infty$ and to $Y_{2}$ as $t \rightarrow \infty$, in a metric sense that can be made precise (Gromov-Hausdorff convergence on bounded subsets).
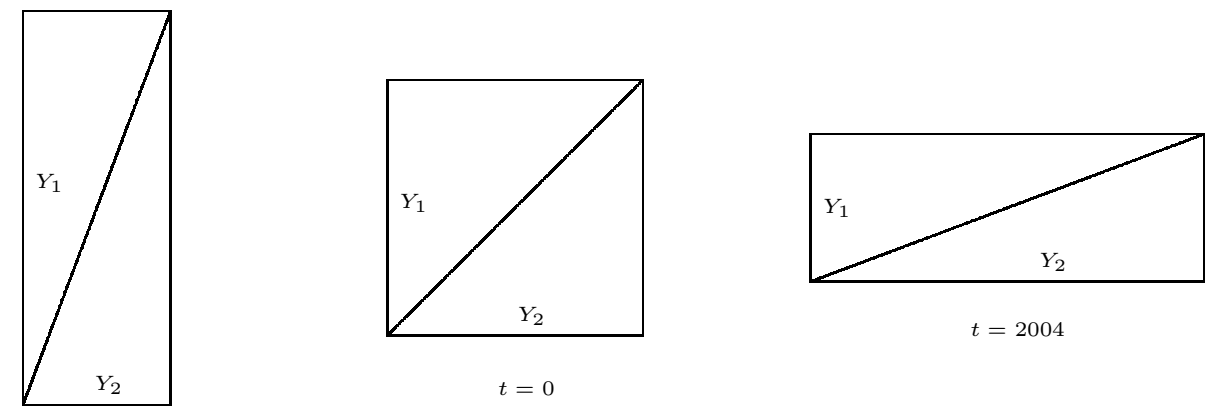

$t=-2004$

Figure 7: The sweep-out $S_{t}$ from $Y_{1}$ to $Y_{2}$, at different times 
$X$ has the natural $\Gamma$-action induced from the $\Gamma$-actions on $Y_{i}$, this provides an isometric $\Gamma$-action on $Y_{1} \otimes Y_{2}$. This action preserves slices and is the diagonal action on each slice $S_{t}=Y_{1} \times Y_{2}$.

When $M_{1}$ and $M_{2}$ are closed aspherical manifolds with the same fundamental group, the Borel conjecture asserts that they are homeomorphic. If there is indeed a homeomorphism, then each slice in $Y_{1} \otimes Y_{2}$ contains a homeomorphic and $\Gamma$-invariant copy of $Y_{1}=Y_{2}$. Thus the asymmetric join is a place to look for homeomorphisms, and the metric $d_{*}$ on it should allow for analytic and geometric tools to be used.

\subsection{Group-theoretic rigidity and the Poincaré conjecture}

The Mostow rigidity theorem 32 implies that the Borel conjecture holds in the case of hyperbolic manifolds: two closed hyperbolic manifolds with the same fundamental group are homeomorphic. This can be viewed as an example of a topological theorem where geometric assumptions are necessary to run the proof.

There is an interesting conjecture which is a topological version of the Mostow rigidity theorem: if two closed aspherical manifolds $M$ and $N$ have the same fundamental group and $N$ is hyperbolic, then $M$ and $N$ are homeomorphic. This was proved by Farrell and Jones [19, 18, 20] in the dimensions other than 3 and 4. Again, geometric assumptions were important for the proof. Farrell and Jones use the dynamics of the geodesic flow on a hyperbolic manifold: the flow shrinks certain paths in the unit tangent bundle. Gabai, Meyerhoff and Thurston 24] showed this conjecture for 3-manifolds under the additional assumption that $M$ is irreducible; it is this assumption that prevents the Poincaré conjecture to be deduced from the result.

From our metric (i.e. non-Riemannian) point of view, the following grouptheoretic version of the conjecture is of interest: if two closed aspherical manifolds have the same fundamental group which is Gromov hyperbolic, then they are homeomorphic. This conjecture is intermediate: it follows from the Borel conjecture and, if true in dimension 3 , it implies the Poincaré conjecture. Theorem [60 provides a construction of a geodesic flow $\mathcal{F}(X)$ with the properties needed: the flow (i.e. the $\mathbb{R}$-action) indeed shrinks distances exponentially. What is missing here is the bundle structure, for the obvious reason: we did not have a manifold to start with, $X$ could be any metric space. It is also worth mentioning that in our construction $\mathbb{R}$ acts by bi-Lipschitz homeomorphisms, so the topology and geometry of the space are preserved. 


\subsection{Index of symbols and terminology}

Symbols and terminology are indexed by section/subsection. Readers interested in the LTEX codes which produce the symbols should consult symbols.tex or symbols.pdf, which can be downloaded from:

\begin{tabular}{|c|c|c|}
\hline \multicolumn{3}{|c|}{ http://msp.warwick.ac.uk/gt/2005/09/x013.xhtml } \\
\hline$X \bowtie X, 2.1$ & $\beta^{\times}, 3.1,8.4,10$ & cross-ratio, 7 \\
\hline$\diamond X, 2.1$ & $\theta, 1.6$ & distance-minimizing \\
\hline$\diamond X, 2.2$ & $\theta^{\prime}, 1.6$ & geodesic, 6.4 \\
\hline$\otimes X, 2.1$ & $\psi=\psi_{X}, 8.5$ & pair, 6.4 \\
\hline$x_{0}^{\circledast} X, 2.2$ & $d=d_{X}, 5.1$ & double difference, 1.16 .2 \\
\hline$Y_{1} \oplus Y_{2}, 14.1$ & $\hat{d}=\hat{d}_{X}, 6.1$ & end-point coordinates, $[2.2$ \\
\hline$\otimes \bar{X}, 8$ & $d^{\times}$in $\otimes X, 3.2$ & flow space, 13 \\
\hline$* X, 2.1$ & $d^{\times}$in $\otimes \bar{X}, 8.6$ & generalized metric, 1.2 \\
\hline$x_{0}^{*} X, \overline{2.4}$ & $d_{*}=\otimes d$ in $X, 3.3$ & geodesic, 5.3 \\
\hline$* \bar{X}, 8.3$ & $d_{*}=\otimes \hat{d}$ in $\bar{X}, 8.6$ & Gromov product, $1.1,6.2$ \\
\hline$* \bar{X}, 8.3$ & $\partial X$-triple, 6.7 & horosphere, [10] \\
\hline $\bar{X}^{\diamond}, 6.7$ & trivial, 6.7 & horofunction in $\oplus \bar{X}, 10$ \\
\hline $\bar{X}^{\triangleright}, 6.7$ & $\partial^{2} X, 13$ & hyperbolic complex, 5.3 \\
\hline$\widetilde{1}, 1.3$ & $\ell(u, x), 3.1$ & hyperbolic graph, 5.3 \\
\hline${ }^{+}$equivalence, 1.3 & $\mathcal{F}(X), 13$ & induced from 0 -skeleton, 5.2 \\
\hline$\times$ equivalence, 1.3 & $\operatorname{Geod}(a, b), 5.3$ & inscribed triple, 5.3 \\
\hline$\times+$ equivalence, 1.3 & $g_{+}, g_{-}, 12$ & isometric embedding, 1.2 \\
\hline + geodesic, 6.6 & $\hat{l}(g), 12$ & metric complex, 5.2 \\
\hline+ isometry, 1.3 & $\mathrm{np}[\alpha \mid y], \operatorname{np}\left[a, a^{\prime} \mid y\right], 6.4$ & metric join, 2.114 .2 \\
\hline$x+$ isometry, 1.3 & $r^{+}, 2.6$ & nearest-point projection, 6 \\
\hline${ }^{+} \operatorname{map}, 1.3$ & $\overline{\mathbb{R}}, 1.4$ & open symmetric join \\
\hline${ }^{\times} \operatorname{map}, 1.3$ & $\mathcal{T}_{*}, 9.1$ & of $X, 2.1$ \\
\hline${ }^{x+} \operatorname{map}, 1.3$ & $U^{+}(a, t), U^{-}(a, t), 6.5$ & of $\bar{X}, 8.3$ \\
\hline$\langle\cdot, \cdot \mid \cdot, \cdot\rangle, 1.16 .2$ & $V^{+}(a, t), V^{-}(a, t), 6.5$ & projection, 2.9] \\
\hline $\begin{array}{l}(\cdot, \cdot \mid \cdot, \cdot), 6.2 \\
\|\cdot \cdot \cdot \cdot \cdot, \cdot\|, 7\end{array}$ & $\llbracket \cdot, \cdot ; \cdot \rrbracket^{\prime}, 2.3$ & $\begin{array}{l}\text { proper metric space, } 9.3 \\
\text { quasiisometry, } 1.3\end{array}$ \\
\hline$\langle a \mid b\rangle_{c}, 1.16 .2$ & asymmetric join, 14 & shift-invariance \\
\hline$(a \mid b)_{c}, 6.2$ & actions on $\otimes X$ & of $\theta$ and $\theta^{\prime}, 1.6$ \\
\hline$\left\langle a, a^{\prime} \mid b\right\rangle,[2.9$ & $\operatorname{Isom}(X)-, 2.8$ & side pair, 6.7 \\
\hline$\llbracket a, a^{\prime} \mid b \rrbracket, 2.9$ & $\mathbb{R}-, 2.6$ & side $\partial X$-pair, 6.7 \\
\hline$\left[S_{1}, S_{2}\right], 6.5$ & $\mathbb{Z}_{2}-, 2.7$ & sweep-out, 14.5 \\
\hline$\left[a, a^{\prime} \mid x_{0}\right], 8.5$ & actions on $\oplus \bar{X}, 8.2$ & symmetric join \\
\hline$\llbracket a, b \rrbracket=\llbracket a, b \rrbracket_{x_{0}}$ & appropriate number, 2.2 & of $X, 2.1$ \\
\hline in $\otimes X, 2.2$ & coordinate & of $\bar{X}, 8$ \\
\hline in $\otimes \bar{X}, 8.1$ & $x_{0}$-coordinate, 2.2 & translation length, 12 \\
\hline$\llbracket a, b ; t \rrbracket$ and $\llbracket a, b ; t \rrbracket^{\prime}$ & of the projection, [2.9] & trivial $\partial X$-triple, 6.7 \\
\hline in $\otimes X, 2.2$ & convexity of $\mathcal{T}_{*}, 9.1$ & word metric $d, 5.15 .3$ \\
\hline
\end{tabular}




\section{References}

[1] N Bourbaki, Общая топология. Основные структуры. (Russian) [General topology. Fundamental structures], Izdat. "Nauka", Moscow (1968), translated from the French by S N Kračkovskiı̆, edited by D A Raǔkov MR0244925

[2] Marc Bourdon, Structure conforme au bord et flot géodésique d'un CAT(-1)espace, Enseign. Math. 41 (1995) 63-102 MR1341941

[3] Marc Bourdon, Sur le birapport au bord des CAT(-1)-espaces, Inst. Hautes Études Sci. Publ. Math. (1996) 95-104 MR1423021

[4] Martin Bridson, André Haefliger, Metric spaces of non-positive curvature, Grundlehren der Mathematischen Wissenschaften 319, Springer-Verlag, Berlin (1999) MR1744486

[5] Theo Bühler, Gromov's geodesic flow, Diploma, ETH (2002)

[6] J W Cannon, The combinatorial Riemann mapping theorem, Acta Math. 173 (1994) 155-234 MR1301392

[7] J W Cannon, Daryl Cooper, A characterization of cocompact hyperbolic and finite-volume hyperbolic groups in dimension three, Trans. Amer. Math. Soc. 330 (1992) 419-431 MR1036000

[8] J W Cannon, EL Swenson, Recognizing constant curvature discrete groups in dimension 3, Trans. Amer. Math. Soc. 350 (1998) 809-849 MR1458317

[9] J W Cannon, William J Floyd, Richard Kenyon, Walter R Parry, $H y$ perbolic geometry, from: "Flavors of geometry", Math. Sci. Res. Inst. Publ. 31, Cambridge Univ. Press, Cambridge (1997) 59-115 MR1491098

[10] Christophe Champetier, Petite simplification dans les groupes hyperboliques, Ann. Fac. Sci. Toulouse Math. (6) 3 (1994) 161-221 MR1283206

[11] T A Chapman, Homotopy conditions which detect simple homotopy equivalences, Pacific J. Math. 80 (1979) 13-46 MR0534693

[12] Ruth M Charney, Michael W Davis, Strict hyperbolization, Topology 34 (1995) 329-350 MR1318879

[13] Michel Coornaert, Mesures de Patterson-Sullivan sur le bord d'un espace hyperbolique au sens de Gromov, Pacific J. Math. 159 (1993) 241-270 MR1214072

[14] Christopher B Croke, Rigidity for surfaces of nonpositive curvature, Comment. Math. Helv. 65 (1990) 150-169 MR1036134

[15] Michael W Davis, Tadeusz Januszkiewicz, Hyperbolization of polyhedra, J. Differential Geom. 34 (1991) 347-388 MR1131435

[16] F T Farrell, L E Jones, $K$-theory and dynamics. I, Ann. of Math. (2) 124 (1986) 531-569 MR0866708

[17] F T Farrell, L E Jones, K-theory and dynamics. II, Ann. of Math. (2) 126 (1987) 451-493 MR0916716 
[18] F T Farrell, L E Jones, Compact negatively curved manifolds (of dim $\neq 3,4$ ) are topologically rigid, Proc. Nat. Acad. Sci. U.S.A. 86 (1989) 3461-3463 MR0997635

[19] F T Farrell, L E Jones, A topological analogue of Mostow's rigidity theorem, J. Amer. Math. Soc. 2 (1989) 257-370 MR0973309

[20] F T Farrell, L E Jones, Rigidity and other topological aspects of compact nonpositively curved manifolds, Bull. Amer. Math. Soc. 22 (1990) 59-64 MR1001606

[21] F T Farrell, L E Jones, Classical aspherical manifolds, CBMS Regional Conference Series in Mathematics 75, Published for the Conference Board of the Mathematical Sciences, Washington, DC (1990) MR1056079

[22] Steve Ferry, The homeomorphism group of a compact Hilbert cube manifold is an ANR, Ann. Math.106 (1977) 101-119 MR0461536

[23] Alex Furman, Coarse-geometric perspective on negatively curved manifolds and groups, from: "Rigidity in dynamics and geometry (Cambridge, 2000)", Springer, Berlin (2002) 149-166 MR1919399

[24] David Gabai, G Robert Meyerhoff, Nathaniel Thurston, Homotopy hyperbolic 3-manifolds are hyperbolic, Ann. of Math. 157 (2003) 335-431 MR1973051

[25] É Ghys, P de la Harpe (editors), Sur les groupes hyperboliques d'après Mikhael Gromov, Papers from the Swiss Seminar on Hyperbolic Groups held in Bern, 1988, Progress in Mathematics 83, Birkhäuser Boston Inc. Boston, MA (1990) MR1086648

[26] É Ghys, A Haefliger, A Verjovsky (editors), Group theory from a geometrical viewpoint, World Scientific Publishing Co. Inc. River Edge, NJ (1991) MR1170362

[27] M Gromov, Hyperbolic groups, from: "Essays in group theory", Math. Sci. Res. Inst. Publ. 8, Springer, New York (1987) 75-263 MR0919829

[28] Sa'ar Hersonsky, Frédéric Paulin, On the rigidity of discrete isometry groups of negatively curved spaces, Comment. Math. Helv. 72 (1997) 349-388 MR1476054

[29] Frédéric Mathéus, Flot géodésique et groupes hyperboliques d'après M. Gromov, from: "Séminaire de Théorie Spectrale et Géométrie, No. 9, Année 19901991", Sémin. Théor. Spectr. Géom. 9, Univ. Grenoble I, Saint (1991) 67-87 MR1715930

[30] Igor Mineyev, Nicolas Monod, Yehuda Shalom, Ideal bicombings for hyperbolic groups and applications, Topology 43 (2004) 1319-1344 MR2081428

[31] Igor Mineyev, Guoliang Yu, The Baum-Connes conjecture for hyperbolic groups, Invent. Math. 149 (2002) 97-122 MR1914618 
[32] G D Mostow, Strong rigidity of locally symmetric spaces, Princeton University Press, Princeton, N.J. (1973), Annals of Mathematics Studies 78, Princeton University Press, Princeton, N.J. (1973) MR0385004

[33] Jean-Pierre Otal, Le spectre marqué des longueurs des surfaces à courbure négative, Ann. of Math. 131 (1990) 151-162 MR1038361

[34] Jean-Pierre Otal, Sur la géometrie symplectique de l'espace des géodésiques d'une variété à courbure négative, Rev. Mat. Iberoamericana 8 (1992) 441-456 MR1202417

[35] Frédéric Paulin, Un groupe hyperbolique est déterminé par son bord, J. London Math. Soc. 54 (1996) 50-74 MR1395067

[36] Frank Quinn, Ends of maps. I, Ann. of Math. 110 (1979) 275-331 MR0549490

[37] E Swenson, Negatively curved groups and related topics (1993), PhD thesis, BYU 